\title{
Light-Cone Expansion of the Dirac Sea in the Presence of Chiral and Scalar Potentials
}

\author{
Felix Finster* \\ Department of Mathematics, Harvard University \\ September/November 1998
}

\begin{abstract}
We study the Dirac sea in the presence of external chiral and scalar/pseudoscalar potentials. In preparation, a method is developed for calculating the advanced and retarded Green's functions in an expansion around the light cone. For this, we first expand all Feynman diagrams and then explicitly sum up the perturbation series. The light-cone expansion expresses the Green's functions as an infinite sum of line integrals over the external potential and its partial derivatives.

The Dirac sea is decomposed into a causal and a non-causal contribution. The causal contribution has a light-cone expansion which is closely related to the light-cone expansion of the Green's functions; it describes the singular behavior of the Dirac sea in terms of nested line integrals along the light cone. The non-causal contribution, on the other hand, is, to every order in perturbation theory, a smooth function in position space.
\end{abstract}

\section{Introduction}

In relativistic quantum mechanics with interaction, the fermionic wave functions $\Psi$ are solutions of a Dirac equation of the form

$$
(i \not \partial+\mathcal{B}-m) \Psi=0
$$

where $\mathcal{B}$ is composed of the classical bosonic potentials. According to the common conception, the Dirac sea of the system is built up of all the negative-energy solutions of the Dirac equation. We can describe it with the so-called fermionic projector $\tilde{P} \mathbb{1}$. On the non-rigorous level of this introduction, the fermionic projector is given by the formal sum of the projectors on all these solutions; i.e.

$$
\tilde{P}(x, y) \stackrel{\text { formally }}{=} \sum_{a} \Psi_{a}(x) \overline{\Psi_{a}(y)},
$$

where the index ' $a$ ' runs over all the quantum numbers of the negative-energy states. We want to analyze how the fermionic projector depends on the bosonic potentials in (1.1). According to the decomposition (1.2) into the individual states, this dependence can be regarded as a collective effect of all the fermions of the Dirac sea moving in the

\footnotetext{
* Mailing address after October 1998: Max-Planck-Institut für Mathematik in den Naturwissenschaften, Inselstr. 22-26, 04103 Leipzig, Germany.
} 
external potential $\mathcal{B}$. Following Dirac's original concept that the completely filled Dirac sea describes the vacuum, we can also say that we are interested in how the fermionic vacuum is influenced by the bosonic fields. Our aim is to describe this physical effect in a detailed and explicit way.

It turns out that the dependence of the fermionic projector on the external potential has a complicated non-local structure. In order to simplify the problem, we shall study $\tilde{P}(x, y)$ in an expansion about the light cone, which is called light-cone expansion. The light cone around a space-time point $x$ consists of all points which can be reached from $x$ with a light ray. In flat Minkowski space, which we will consider here, the light cone is given by all pairs of points $(x, y)$ of Minkowski space whose Lorentzian distance $(y-x)^{2} \equiv(y-x)_{j}(y-x)^{j}$ is zero. In the simplest case of a smooth function $A(x, y)$, the light-cone expansion is just an expansion in powers of $(y-x)^{2}$, i.e. a representation of the form

$$
A(x, y)=\sum_{j=0}^{\infty}(y-x)^{2 j} A_{j}(x, y)
$$

with smooth functions $A_{j}(x, y)$. Since the expansion parameter $(y-x)^{2}$ vanishes on the light cone, the coefficients $A_{j}(x, y)$ give approximations of $A(x, y)$ in a neighborhood of the light cone (i.e., $A_{0}(x, y)$ coincides with $A(x, y)$ on the light cone, $A_{1}(x, y)$ gives the first order behavior of $A(x, y)$ for pairs $(x, y)$ which are close to the light cone, etc.). The important point is that the $A_{j}(x, y)$ are approximations of $A(x, y)$ even for points $x, y$ which are far apart. We only need that the pair $(x, y)$ is close to the light cone, which is an unbounded hypersurface in $\mathbb{R}^{4} \times \mathbb{R}^{4}$. In this sense, the light-cone expansion is a non-local expansion. The major advantage over local approximation techniques (like e.g. Taylor expansions in the space-time coordinates) is that the light-cone expansion gives a much more detailed description of the fermionic projector in position space. Furthermore, since the light cone is the boundary of the domain of causal dependence, all the effects related to the causality of the Dirac equation occur near the light cone. Thus the lightcone expansion describes the fermionic projector precisely in the region which is most interesting physically. In this paper, we will develop an efficient method for performing the light-cone expansion of the fermionic projector.

After this simplified and very qualitative introduction, we briefly discuss the difficulties and methods of the more detailed study. First of all, it is not obvious how to characterize the "negative-energy solutions" of the Dirac equation in the case with general interaction. In other words, one problem is to find the right quantum numbers for the $a$-summation in (1.2). As explained in [1], this problem can only be solved if the notion of "negativeenergy states" is given up and replaced by a causality principle for the Dirac sea; this gives a unique definition of $\tilde{P}$ in terms of a power series in the external potential. Our task is to convert this formal definition into explicit formulas for the fermionic projector in position space. The basic technique is to construct solutions of the inhomogeneous Klein-Gordon and Dirac equations and to show that these solutions coincide with the contributions to the perturbation expansion of $\tilde{P}$. For the contribution to first order in the external potential, a similar technique was already used in [2], which also contains a general discussion of the method. In the following, we will first generalize this technique to higher order perturbation theory. Then we will explicitly sum up the light-cone expansions of all Feynman diagrams, which will finally yield exact formulas for the light-cone expansion of the fermionic projector without the restriction for the external potential to be (in any sense) "small." We shall use the notation, the definitions, and the results of [1] throughout. 
Since the method of [2] had to be refined considerably for the analysis of higher order Feynman diagrams, we will develop the light-cone expansion from the very beginning. Thus this paper can be considered as being independent of [2]. Nevertheless, the more elementary approach in [2] is a preparation and might be helpful for the understanding. We will use the so-called residual argument to deduce the light-cone expansion of the Dirac sea from that of the advanced and retarded Green's functions. This allows us to bypass the explicit Fourier transformations in [2]. However, the residual argument has its limitations; making it mathematically precise leads to the decomposition of the Dirac sea into a causal and a non-causal contribution.

In the remainder of this section, we specify our problem in mathematical terms. Since a realistic physical system consists of several types of fermions, we describe empty space by the fermionic projector of the vacuum, which was introduced in [1] as the direct sum of $f \geq 1$ Dirac seas. The chiral asymmetry matrix $X$ and the mass matrix $Y$ are considered as a-priori given. The reader who is only interested in the light-cone expansion of a single Dirac sea may specialize to $f=1$ and $X=\mathbb{1}=Y$. On the wave functions, we consider the indefinite scalar product

$$
<\Psi \mid \Phi>=\int_{\mathbb{R}^{4}} \sum_{l=1}^{f} \overline{\Psi_{l}(x)} \Phi_{l}(x) d^{4} x
$$

with the adjoint spinor $\bar{\Psi}=\Psi^{*} \gamma^{0}$. Similar to (1.1), the interaction is described by a perturbation $\mathcal{B}$ of the Dirac operator. We allow $\mathcal{B}$ to be composed of chiral and scalar/pseudoscalar potentials,

$$
\mathcal{B}(x)=\chi_{L} A_{R}(x)+\chi_{R} A_{L}(x)+\Phi(x)+i \gamma^{5} \Xi(x),
$$

where $\chi_{L / R}=\frac{1}{2}\left(\mathbb{1} \mp \gamma^{5}\right)$ are the chiral projectors and where we use a matrix notation in the Dirac sea index,

$$
A_{L / R}=\left(A_{L / R m}^{l}\right)_{l, m=1, \ldots, f} \quad, \quad \Phi=\left(\Phi_{m}^{l}\right)_{l, m=1, \ldots, f} \quad, \quad \Xi=\left(\Xi_{m}^{l}\right)_{l, m=1, \ldots, f}
$$

(so the potentials may be non-diagonal on the Dirac seas and thus describe a general interaction of all the fermions). Furthermore, the perturbation $\mathcal{B}$ shall be Hermitian with respect to the scalar product (1.4). We assume the Dirac operator to be causality compatible with $X$, i.e.

$$
X^{*}(i \not \partial+\mathcal{B}-m Y)=(i \not \partial+\mathcal{B}-m Y) X
$$

This assumption is crucial; if it was violated, unbounded line integrals would occur in the light-cone expansion, making it impossible to carry out the sum over all Feynman diagrams (see the calculations in [3] for more details). The form of the chiral decomposition $\chi_{L} A_{R}+\chi_{R} A_{L}$ is useful because, as we will see later, the left and right handed components of the fermions couple to $A_{L}$ and $A_{R}$, respectively. An interesting feature of our system is that, as a consequence of the non-diagonal form (1.6) of the potentials on the Dirac seas, the potentials and the mass matrix do in general not commute with each other,

$$
\left[A_{L}(x), A_{L / R}(y)\right] \neq 0 \quad, \quad\left[A_{L}(x), \Phi(y)\right] \neq 0 \quad, \quad\left[A_{L}(x), Y\right] \neq 0, \ldots \quad .
$$

Compared to the situation in [2], the bilinear potential $H_{j k} \sigma^{j k}$ is missing in (1.5). It leads to complications when the sum over all Feynman diagrams is carried out. These 
complications are not serious, but in order to keep the expansion reasonably simple, the bilinear potential was left out. Furthermore, we do not consider the gravitational field. The reason is that the higher order contributions in the gravitational potential become more and more singular on the light cone. This leads to technical problems which we will not deal here. Despite these simplifications, the considered ansatz for $\mathcal{B}$ includes arbitrary left and right handed Yang-Mills potentials and is general enough for a description of e.g. the interactions of the standard model. The fermionic projector in the presence of external fields, $\tilde{P}(x, y)$, is defined via the perturbation series in [1], which is a formal sum of operator products of the form

$$
\tilde{P}=\sum_{n=0}^{\infty} \sum_{\ldots} \operatorname{const}(n, \ldots) C_{n, \ldots} \mathcal{B} C_{n-1, \ldots} \mathcal{B} \cdots \mathcal{B} C_{0, \ldots},
$$

where the factors $C_{j, \ldots}$ coincide either with the spectral projectors $k, p$, or with the Green's function $s$ (the index '...' is a short notation for the different configurations of these factors).

In the language of Feynman diagrams, the perturbation series for $\tilde{P}$ only consists of tree diagrams. These tree diagrams are all finite; this is not difficult to prove if we assume suitable regularity of the potential:

Lemma 1.1 Let $\left(C_{j}\right), 0 \leq j \leq n$, be a choice of operators $C_{j}=k, p$, or $s$. If the external potential (1.5) is smooth and decays so fast at infinity that the functions $\mathcal{B}(x), x^{i} \mathcal{B}(x)$, and $x^{i} x^{j} \mathcal{B}(x)$ are integrable, then the operator product

$$
\left(C_{n} \mathcal{B} C_{n-1} \mathcal{B} \cdots \mathcal{B} C_{0}\right)(x, y)
$$

is a well-defined tempered distribution on $\mathbb{R}^{4} \times \mathbb{R}^{4}$.

Proof: Calculating the Fourier transform of (1.8) gives the formal expression

$$
\begin{aligned}
& M\left(q_{2}, q_{1}\right):=\int \frac{d^{4} p_{1}}{(2 \pi)^{4}} \cdots \int \frac{d^{4} p_{n-1}}{(2 \pi)^{4}} \\
& \quad \times C_{n}\left(q_{2}\right) \tilde{\mathcal{B}}\left(q_{1}-p_{n-1}\right) C_{n-1}\left(p_{n-1}\right) \tilde{\mathcal{B}}\left(p_{n-1}-p_{n-2}\right) \cdots C_{1}\left(p_{1}\right) \tilde{\mathcal{B}}\left(p_{1}-q_{1}\right) C_{0}\left(q_{1}\right),
\end{aligned}
$$

where we consider the $C_{j}$ as multiplication operators in momentum space and where $\tilde{\mathcal{B}}$ denotes the Fourier transform of $\mathcal{B}$. It is more convenient to work in momentum space because the operators $C_{j}$ then have a simpler form. We will show that $M\left(q_{2}, q_{1}\right)$ is a well-defined tempered distribution; our Lemma then immediately follows by transforming back to position space.

The assumptions on $\mathcal{B}$ yield that $\tilde{\mathcal{B}}$ is $C^{2}$ and has rapid decay at infinity, i.e.

$$
\sup _{q \in \mathbb{R}^{4},|\kappa| \leq 2}\left|q^{i_{1}} \cdots q^{i_{n}} \partial_{\kappa} \tilde{\mathcal{B}}(q)\right|<\infty
$$

for all $n$, tensor indices $i_{1}, \ldots, i_{n}$, and multi-indices $\kappa\left(\right.$ so $\left.\kappa=\left(\kappa^{1}, \ldots, \kappa^{p}\right),|\kappa|:=p\right)$. As is verified explicitly in momentum space, the distributions $k, p$, and $s$ are bounded in the Schwartz norms of the test functions involving derivatives of only first order, more precisely

$$
|C(f)| \leq \text { const }\|f\|_{4,1} \quad \text { with } C=k, p \text {, or } s \text { and } f \in \mathcal{S},
$$

where the Schwartz norms are as usual defined by

$$
\|f\|_{p, q}=\max _{|I| \leq p,|J| \leq q} \sup _{x \in \mathbb{R}^{4}}\left|x^{I} \partial_{J} f(x)\right| .
$$


As a consequence, we can apply $k, p$, and $s$ even to functions with rapid decay which are only $C^{1}$. Furthermore, we can form the convolution of such functions with $k, p$, or $s$; this gives continuous functions (which will no longer have rapid decay, however). A convolution decreases the order of differentiability of the functions by one.

We consider the combination of multiplication and convolution

$$
F\left(p_{2}\right):=\int \frac{d^{4} p_{1}}{(2 \pi)^{4}} f\left(p_{2}-p_{1}\right) C\left(p_{1}\right) g\left(p_{1}\right)
$$

where we assume that $f \in C^{2}$ has rapid decay and $g \in C^{1}$ is bounded together with its first derivatives, $\|g\|_{0,1}<\infty$. For any fixed $p_{2}$, the integral in (1.10) is well-defined and finite because $f\left(p_{2}-.\right) g($.$) is C^{1}$ and has rapid decay. The resulting function $F$ is $C^{1}$ and bounded together with its first derivatives, more precisely

$$
\|F\|_{0,1} \leq \text { const }\|f\|_{4,2}\|g\|_{0,1}
$$

After these preparations, we can estimate the integrals in (1.9) from the right to the left: We choose two test functions $f, g \in \mathcal{S}\left(\mathbb{R}^{4}, \mathbb{C}^{4 f}\right)$ and introduce the functions

$$
\begin{aligned}
& F_{1}\left(p_{1}\right)=\int \frac{d^{4} q_{2}}{(2 \pi)^{4}} \tilde{\mathcal{B}}\left(p_{1}-q_{1}\right) C_{0}\left(q_{1}\right) g\left(q_{1}\right) \\
& F_{j}\left(p_{j}\right)=\int \frac{d^{4} p_{j-1}}{(2 \pi)^{4}} \tilde{\mathcal{B}}\left(p_{j}-p_{j-1}\right) C_{j-1}\left(p_{j-1}\right) F_{j-1}\left(p_{j-1}\right), \quad 1<j \leq n .
\end{aligned}
$$

The integral (1.12) is of the form (1.10) and satisfies the above considered assumptions on the integrand. Using the bound (1.11), we can proceed inductively in (1.13). Finally, we perform the $q_{2}$-integration,

$$
M(f, g)=\int \frac{d^{4} q_{2}}{(2 \pi)^{4}} f\left(q_{2}\right) C_{n}\left(q_{2}\right) F_{n}\left(q_{2}\right)
$$

We conclude that $M$ is a linear functional on $\mathcal{S}\left(\mathbb{R}^{4}, \mathbb{C}^{4 f}\right) \times \mathcal{S}\left(\mathbb{R}^{4}, \mathbb{C}^{4 f}\right)$, which is bounded in the Schwartz norm $\|\cdot\|_{4,1}$ of the test functions.

Clearly, the existence of the perturbation expansion to every order does not imply the convergence of the perturbation series, and we will not study this problem here. Our method is to first perform the light-cone expansion of the individual Feynman diagrams. For the resulting formulas, it will then be possible to sum up the perturbation series. Since the Feynman diagrams are only defined as distributions, we must generalize (1.3) in a way which allows for the possibility that $A(x, y)$ is singular on the light cone.

Def. 1.2 A tempered distribution $A(x, y)$ is of the order $\mathcal{O}\left((y-x)^{2 p}\right), p \in \mathbf{Z}$, if the product

$$
(y-x)^{-2 p} A(x, y)
$$

is a regular distribution (i.e. a locally integrable function). It has the light-cone expansion

$$
A(x, y)=\sum_{j=g}^{\infty} A^{[j]}(x, y)
$$


with $g \in \mathbf{Z}$ if the distributions $A^{[j]}(x, y)$ are of the order $\mathcal{O}\left((y-x)^{2 j}\right)$ and if $A$ is approximated by the partial sums in the way that

$$
A(x, y)-\sum_{j=g}^{p} A^{[j]}(x, y) \quad \text { is of the order } \quad \mathcal{O}\left((y-x)^{2 p+2}\right)
$$

for all $p \geq g$.

The lowest summand $A^{[g]}(x, y)$ gives the leading order of $A(x, y)$ on the light cone. If $A$ is singular on the light cone, $g$ will be negative. The light-cone expansion (1.3) of a smooth function is recovered as a special case by setting $g=0$ and $A^{[j]}(x, y)=(y-x)^{2 j} A_{j}(x, y)$. Notice that the definition of the light-cone expansion does not include the convergence of the infinite sum in (1.14), which is only a convenient notation for the approximation of $A(x, y)$ by the partial sums (1.15).

\section{The Light-Cone Expansion of the Green's Functions}

In this section, we shall perform the light-cone expansion for the advanced and retarded Green's functions $\tilde{s}^{\vee}, \tilde{s}^{\wedge}$, which are defined in [1] by the perturbation series

$$
\tilde{s}^{\vee}=\sum_{k=0}^{\infty}\left(-s^{\vee} \mathcal{B}\right)^{k} s^{\vee}, \quad \tilde{s}^{\wedge}=\sum_{k=0}^{\infty}\left(-s^{\wedge} \mathcal{B}\right)^{k} s^{\wedge} .
$$

These perturbation expansions are causal in the sense that $\tilde{s}^{\vee}(x, y)$ and $\tilde{s}^{\wedge}(x, y)$ only depend on the potential $\mathcal{B}$ in the "diamond" $\left(L_{x}^{\vee} \cap L_{y}^{\wedge}\right) \cup\left(L_{x}^{\wedge} \cap L_{y}^{\vee}\right)$, where

$$
L_{x}^{\vee}=\left\{y \mid(y-x)^{2} \geq 0, y^{0}-x^{0} \geq 0\right\}, \quad L_{x}^{\wedge}=\left\{y \mid(y-x)^{2} \geq 0, y^{0}-x^{0} \leq 0\right\}
$$

denote the future and past light cones around $x$, respectively. Since this "diamond" is (for fixed $x$ and $y$ ) a bounded region of space-time, the assumptions of Lemma 1.1 on the decay of the external potential at infinity are not necessary in this section; it suffices to assume that $\mathcal{B}$ is smooth. Furthermore, the chiral asymmetry matrix $X$ and the causality compatibility condition will not be needed in this section.

In order to get a first idea of how the light-cone expansion can be carried out, we look at the free advanced Green's function $s_{m}^{\vee}$ of a single Dirac sea in position space: It is convenient to pull the Dirac matrices out of $s_{m}^{\vee}$ by setting

$$
s_{m}^{\vee}(x, y)=\left(i \not \partial_{x}+m\right) S_{m^{2}}^{\vee}(x, y),
$$

where $S_{m^{2}}^{\vee}$ is the advanced Green's function of the Klein-Gordon operator,

$$
S_{m^{2}}^{\vee}(x, y)=\lim _{0<\varepsilon \rightarrow 0} \int \frac{d^{4} p}{(2 \pi)^{4}} \frac{1}{p^{2}-m^{2}-i \varepsilon p^{0}} e^{-i p(x-y)} .
$$

This Fourier integral can be calculated explicitly; we expand the resulting Bessel function $J_{1}$ in a power series,

$$
\begin{aligned}
S_{m^{2}}^{\vee}(x, y)= & -\frac{1}{2 \pi} \delta\left((y-x)^{2}\right) \Theta\left(y^{0}-x^{0}\right) \\
& +\frac{m^{2}}{4 \pi} \frac{J_{1}\left(\sqrt{m^{2}(y-x)^{2}}\right.}{\sqrt{m^{2}(y-x)^{2}}} \Theta\left((y-x)^{2}\right) \Theta\left(y^{0}-x^{0}\right)
\end{aligned}
$$




$$
\begin{aligned}
= & -\frac{1}{2 \pi} \delta\left((y-x)^{2}\right) \Theta\left(y^{0}-x^{0}\right) \\
& +\frac{m^{2}}{8 \pi} \sum_{j=0}^{\infty} \frac{(-1)^{j}}{j !(j+1) !} \frac{\left(m^{2}(y-x)^{2}\right)^{j}}{4^{j}} \Theta\left((y-x)^{2}\right) \Theta\left(y^{0}-x^{0}\right)
\end{aligned}
$$

( $\Theta$ is the Heaviside function $\Theta(t)=1$ for $t \geq 0$ and $\Theta(t)=0$ otherwise). This computation shows that $S_{m^{2}}^{\vee}(x, y)$ has a $\delta\left((y-x)^{2}\right)$-like singularity on the light cone. Furthermore, one sees that $S_{m^{2}}^{\vee}$ is a power series in $m^{2}$. The important point for the following is that the higher order contributions in $m^{2}$ contain more factors $(y-x)^{2}$ and are thus of higher order on the light cone. More precisely,

$$
\left(\frac{d}{d m^{2}}\right)^{n} S_{m^{2} \mid m^{2}=0}^{\vee}(x, y) \quad \text { is of the order } \quad \mathcal{O}\left((y-x)^{2 n-2}\right) .
$$

According to (2.3), the Dirac Green's function is obtained by computing the first partial derivatives of (2.5). Therefore $s_{m}^{\vee}(x, y)$ has a singularity on the light cone which is even $\sim \delta^{\prime}\left((y-x)^{2}\right)$. The higher order contributions in $m$ are again of increasing order on the light cone. This means that we can view the Taylor expansion of (2.3) in $m$,

$$
s_{m}^{\vee}(x, y)=\sum_{n=0}^{\infty}(i \not \partial+m) \frac{1}{n !}\left(\frac{d}{d m^{2}}\right)^{n} S_{m^{2} \mid m^{2}=0}^{\vee}(x, y)
$$

as a light-cone expansion of the free Green's function. Our idea is to generalize this formula to the case with interaction. More precisely, we want to express the perturbed Green's function in the form

$$
\tilde{s}^{\vee}(x, y)=\sum_{n=0}^{\infty} F_{n}(x, y)\left(\frac{d}{d m^{2}}\right)^{n} S_{m^{2} \mid m^{2}=0}^{\vee}(x, y)
$$

with factors $F_{n}$ which depend on the external potential. We will see that this method is very convenient; especially, we can in this way avoid working with the rather complicated explicit formula (2.5). Apart from giving a motivation for the desired form (2.7) of the formulas of the light-cone expansion, the mass expansion (2.5) leads to the conjecture that even the higher order contributions in the mass to the perturbed Green's functions might be of higher order on the light cone. If this conjecture was true, it would be a good idea to expand the perturbation expansion for $\tilde{s}$ with respect to the parameter $m$. Therefore our basic strategy is to first expand (2.1) with respect to the mass and to try to express the contributions to the resulting expansion in a form similar to (2.7).

The expansion of (2.1) with respect to $m$ gives a double sum over the orders in the mass parameter and in the external potential. It is convenient to combine these two expansions in a single perturbation series. For this, we first write the mass matrix and the scalar/pseudoscalar potentials together by setting

$$
Y_{L}(x)=Y-\frac{1}{m}(\Phi(x)+i \Xi(x)), \quad Y_{R}(x)=Y-\frac{1}{m}(\Phi(x)-i \Xi(x)) .
$$

The matrices $Y_{L / R}(x)$ are called dynamic mass matrices; notice that $Y_{L}^{*}=Y_{R}$. With this notation, we can rewrite the Dirac operator in the form

$$
\begin{aligned}
i \not \partial+\mathcal{B}-m Y & =i \not \partial+B \quad \text { with } \\
B & =\chi_{L}\left(A_{R}-m Y_{R}\right)+\chi_{R}\left(A_{L}-m Y_{L}\right) .
\end{aligned}
$$


For the light-cone expansion of the Green's functions, we will always view $B$ as the perturbation of the Dirac operator. This has the advantage that the free theory consists only of zero-mass fermions; the Green's functions of the free Dirac operator have the simple form

$$
s^{\vee}(x, y)=i \not \partial_{x} S_{m^{2}=0}^{\vee}(x, y), \quad s^{\wedge}(x, y)=i \not \partial_{x} S_{m^{2}=0}^{\wedge}(x, y)
$$

(to be very precise, we should write $s^{\vee}=i \not \partial S_{0}^{\vee} \otimes \mathbb{1}$, where $\mathbb{1}=\left(\mathbb{1}_{m}^{l}\right)_{l, m=1, \ldots, f}$ is the identity on the Dirac seas). The Green's functions with interaction are given by the perturbation series

$$
\tilde{s}^{\vee}=\sum_{k=0}^{\infty}\left(-s^{\vee} B\right)^{k} s^{\vee}, \quad \tilde{s}^{\wedge}=\sum_{k=0}^{\infty}\left(-s^{\wedge} B\right)^{k} s^{\wedge} .
$$

The constructions of the following subsections are exactly the same for the advanced and retarded Green's functions. In order to treat both cases at once, we will in the remainder of this section omit all superscripts ${ }^{\vee} \vee,{ }^{\circ},{ }^{\wedge}$ '. The formulas for the advanced and retarded Green's functions are obtained by either adding ' $\vee$ ' or ' $\wedge$ ' to all factors $s, S$.

\subsection{Inductive Light-Cone Expansion of All Feynman Diagrams}

In this subsection, we explain how the individual contributions to the perturbation expansion (2.12) can be written similar to the right side of (2.7) as a sum of terms of increasing order on the light cone. For the mass expansion of $S_{m^{2}}$, we set $a=m^{2}$ and use the notation

$$
S^{(l)}=\left(\frac{d}{d a}\right)^{l} S_{a \mid a=0}
$$

In preparation, we derive some computation rules for the $S^{(l)}: S_{a}$ satisfies the defining equation of a Klein-Gordon Green's function

$$
\left(-\square_{x}-a\right) S_{a}(x, y)=\delta^{4}(x-y)
$$

Differentiating with respect to $a$ yields

$$
-\square_{x} S^{(l)}(x, y)=\delta_{l, 0} \delta^{4}(x-y)+l S^{(l-1)}(x, y) \quad, \quad l \geq 0 .
$$

(For $l=0$, this formula does not seem to make sense because $S^{(-1)}$ is undefined. The expression is meaningful, however, if one keeps in mind that in this case the factor $l$ is zero, and thus the whole second summand vanishes. We will also use this convention in the following calculations.) Next, we differentiate the formulas for $S_{a}$ in momentum space,

$$
S_{a}^{\vee}(p)=\frac{1}{p^{2}-a-i \varepsilon p^{0}} \quad, \quad S_{a}^{\wedge}(p)=\frac{1}{p^{2}-a+i \varepsilon p^{0}},
$$

with respect to both $p$ and $a$. Comparing the results gives the relation

$$
\frac{\partial}{\partial p^{k}} S_{a}(p)=-2 p_{k} \frac{d}{d a} S_{a}(p)
$$

or, after expanding in the parameter $a$,

$$
\frac{\partial}{\partial p^{k}} S^{(l)}(p)=-2 p_{k} S^{(l+1)}(p) \quad, \quad l \geq 0 .
$$


This formula also determines the derivatives of $S^{(l)}$ in position space; namely

$$
\begin{aligned}
& \frac{\partial}{\partial x^{k}} S^{(l)}(x, y)=\int \frac{d^{4} p}{(2 \pi)^{4}} S^{(l)}(p)\left(-i p_{k}\right) e^{-i p(x-y)} \\
& \stackrel{(2.16)}{=} \frac{i}{2} \int \frac{d^{4} p}{(2 \pi)^{4}} \frac{\partial}{\partial p^{k}} S^{(l-1)}(p) e^{-i p(x-y)}=-\frac{i}{2} \int \frac{d^{4} p}{(2 \pi)^{4}} S^{(l-1)}(p) \frac{\partial}{\partial p^{k}} e^{-i p(x-y)} \\
& \quad=\frac{1}{2}(y-x)_{k} S^{(l-1)}(x, y) \quad, \quad l \geq 1 .
\end{aligned}
$$

We iterate this relation to calculate the Laplacian,

$$
\begin{aligned}
-\square_{x} S^{(l)}(x, y) & =-\frac{1}{2} \frac{\partial}{\partial x^{k}}\left((y-x)^{k} S^{(l-1)}(x, y)\right) \\
& =2 S^{(l-1)}(x, y)-\frac{1}{4}(y-x)^{2} S^{(l-2)}(x, y) \quad, \quad l \geq 2 .
\end{aligned}
$$

After comparing with 2.14, we conclude that

$$
(y-x)^{2} S^{(l)}(x, y)=-4 l S^{(l+1)}(x, y) \quad, \quad l \geq 0 .
$$

Finally, $S^{(l)}(x, y)$ is only a function of $(y-x)$, which implies that

$$
\frac{\partial}{\partial x^{k}} S^{(l)}(x, y)=-\frac{\partial}{\partial y^{k}} S^{(l)}(x, y) \quad, \quad l \geq 0 .
$$

The following lemma gives the light-cone expansion of an operator product which is linear in the external potential. We will later use it for the iterative light-cone expansion of more complicated operator products; in this case, the potential will be a composite expression in $B$ and its partial derivatives. In order to avoid confusion then, we denote the external potential by $V$.

Lemma 2.1 (light-cone expansion to first order) The operator product $S^{(l)} V S^{(r)}$ with $l, r \geq 0$ has the light-cone expansion

$$
\begin{aligned}
& \left(S^{(l)} V S^{(r)}\right)(x, y) \\
& \quad=\sum_{n=0}^{\infty} \frac{1}{n !} \int_{0}^{1} \alpha^{l}(1-\alpha)^{r}\left(\alpha-\alpha^{2}\right)^{n}\left(\square^{n} V\right)_{\mid \alpha y+(1-\alpha) x} d \alpha S^{(n+l+r+1)}(x, y)
\end{aligned}
$$

Proof: The method of the proof is to first compute the Laplacian of both sides of (2.20). The resulting formulas will be of similar structure, which allows us to proceed inductively.

On the left side of (2.20), we calculate the Laplacian with the help of (2.14) to

$$
-\square_{x}\left(S^{(l)} V S^{(r)}\right)(x, y)=\delta_{l, 0} V(x) S^{(r)}(x, y)+l\left(S^{(l-1)} V S^{(r)}\right)(x, y) \quad .
$$

The Laplacian of the integral on the right side of $(2.20)$ can be computed with $(2.17)$ and (2.14),

$$
\begin{aligned}
&- \square_{x} \\
& \int_{0}^{1} \alpha^{l}(1-\alpha)^{r}\left(\alpha-\alpha^{2}\right)^{n}\left(\square^{n} V\right)_{\mid \alpha y+(1-\alpha) x} d \alpha S^{(n+l+r+1)}(x, y) \\
&=--\int_{0}^{1} \alpha^{l}(1-\alpha)^{r+2}\left(\alpha-\alpha^{2}\right)^{n}\left(\square^{n+1} V\right)_{\mid \alpha y+(1-\alpha) x} d \alpha S^{(n+l+r+1)}(x, y) \\
&-\int_{0}^{1} \alpha^{l}(1-\alpha)^{r+1}\left(\alpha-\alpha^{2}\right)^{n}\left(\partial_{k} \square^{n} V\right)_{\mid \alpha y+(1-\alpha) x} d \alpha(y-x)^{k} S^{(n+l+r)}(x, y) \\
&+(n+l+r+1) \int_{0}^{1} \alpha^{l}(1-\alpha)^{r}\left(\alpha-\alpha^{2}\right)^{n}\left(\square^{n} V\right)_{\mid \alpha y+(1-\alpha) x} d \alpha S^{(n+l+r)}(x, y)
\end{aligned}
$$


In the second summand, we rewrite the partial derivative as a derivative with respect to $\alpha$ and integrate by parts,

$$
\begin{aligned}
\int_{0}^{1} \alpha^{l} & (1-\alpha)^{r+1}\left(\alpha-\alpha^{2}\right)^{n}\left(\partial_{k} \square^{n} V\right)_{\mid \alpha y+(1-\alpha) x} d \alpha(y-x)^{k} \\
= & \int_{0}^{1} \alpha^{l}(1-\alpha)^{r+1}\left(\alpha-\alpha^{2}\right)^{n} \frac{d}{d \alpha}\left(\square^{n} V\right)_{\mid \alpha y+(1-\alpha) x} d \alpha \\
= & -\delta_{n, 0} \delta_{l, 0} V(x)-(n+l) \int_{0}^{1} \alpha^{l}(1-\alpha)^{r+2}\left(\alpha-\alpha^{2}\right)^{n-1}\left(\square^{n} V\right)_{\mid \alpha y+(1-\alpha) x} d \alpha \\
& +(n+r+1) \int_{0}^{1} \alpha^{l}(1-\alpha)^{r}\left(\alpha-\alpha^{2}\right)^{n}\left(\square^{n} V\right)_{\mid \alpha y+(1-\alpha) x} d \alpha \\
= & -\delta_{n, 0} \delta_{l, 0} V(x) \\
& -n \int_{0}^{1} \alpha^{l}(1-\alpha)^{r+2}\left(\alpha-\alpha^{2}\right)^{n-1}\left(\square^{n} V\right)_{\mid \alpha y+(1-\alpha) x} d \alpha \\
& +(n+l+r+1) \int_{0}^{1} \alpha^{l}(1-\alpha)^{r}\left(\alpha-\alpha^{2}\right)^{n}\left(\square^{n} V\right)_{\mid \alpha y+(1-\alpha) x} d \alpha \\
& -l \int_{0}^{1} \alpha^{l-1}(1-\alpha)^{r}\left(\alpha-\alpha^{2}\right)^{n}\left(\square^{n} V\right)_{\mid \alpha y+(1-\alpha) x} d \alpha .
\end{aligned}
$$

We substitute back into the original equation and obtain

$$
\begin{aligned}
(2.22)= & \delta_{n, 0} \delta_{l, 0} V(x) S^{(r)}(x, y) \\
& +l \int_{0}^{1} \alpha^{l-1}(1-\alpha)^{r}\left(\alpha-\alpha^{2}\right)^{n}\left(\square^{n} V\right)_{\mid \alpha y+(1-\alpha) x} d \alpha S^{(n+l+r)}(x, y) \\
& -\int_{0}^{1} \alpha^{l}(1-\alpha)^{r+2}\left(\alpha-\alpha^{2}\right)^{n}\left(\square^{n+1} V\right)_{\mid \alpha y+(1-\alpha) x} d \alpha S^{(n+l+r+1)}(x, y) \\
& +n \int_{0}^{1} \alpha^{l}(1-\alpha)^{r+2}\left(\alpha-\alpha^{2}\right)^{n-1}\left(\square^{n} V\right)_{\mid \alpha y+(1-\alpha) x} d \alpha S^{(n+l+r)}(x, y)
\end{aligned}
$$

After dividing by $n$ ! and summation over $n$, the last two summands are telescopic and cancel each other. Thus one gets

$$
\begin{aligned}
& -\square \sum_{n=0}^{\infty} \frac{1}{n !} \int_{0}^{1} \alpha^{l}(1-\alpha)^{r}\left(\alpha-\alpha^{2}\right)^{n}\left(\square^{n} V\right)_{\mid \alpha y+(1-\alpha) x} d \alpha S^{(n+l+r+1)}(x, y) \\
& =\quad \delta_{l, 0} V(x) S^{(r)}(x, y) \\
& \quad+l \sum_{n=0}^{\infty} \frac{1}{n !} \int_{0}^{1} \alpha^{l-1}(1-\alpha)^{r}\left(\alpha-\alpha^{2}\right)^{n}\left(\square^{n} V\right)_{\mid \alpha y+(1-\alpha) x} d \alpha S^{(n+l+r)}(x, y)
\end{aligned}
$$

We now compare the formulas (2.21) and (2.23) for the Laplacian of both sides of (2.20). In the special case $l=0$, these formulas coincide, and we can use a uniqueness argument for the solutions of the wave equation to prove (2.20): We assume that we consider the advanced Green's function (for the retarded Green's function, the argument is analogous). For given $y$, we denote the difference of both sides of (2.20) by $F(x)$. Since the support of $F(x)$ is in the past light cone $x \in L_{y}^{\wedge}, F$ vanishes in a neighborhood of the hypersurface $\mathcal{H}=\left\{z \in \mathbb{R}^{4} \mid z^{0}=y^{0}+1\right\}$. Moreover, the Laplacian of $F$ is identically zero according to (2.21) and (2.23). We conclude that

$$
\square F=0 \quad \text { and } \quad F_{\mid \mathcal{H}}=\partial_{k} F_{\mid \mathcal{H}}=0 .
$$


Since the wave equation has a unique solution for given initial data on the Cauchy surface $\mathcal{H}, F$ vanishes identically.

The general case follows by induction in $l$ : Suppose that (2.20) holds for given $\hat{l}$ (and arbitrary $r$ ). Then, according to (2.21), (2.23), and the induction hypothesis, the Laplacian of both sides of $(2.20)$ coincides for $l=\hat{l}+1$. The above uniqueness argument for the solutions of the wave equation again gives (2.20).

We recall for clarity that, according to (2.6), the higher $a$-derivatives of $S_{a}(x, y)$ are of higher order on the light cone. Thus the summands in (2.20) are of increasing order on the light cone, and the infinite sum makes mathematical sense in terms of Def. 1.2 via the approximation by the partial sums (1.15).

The representation (2.20) of an operator product as an infinite series of line integrals has some similarity with the formal light-cone expansion [2, Theorem 3.3]. In order to make it easier for the reader to see the connection between these two expansions, we briefly discuss the analogy and the differences: First of all, we are here considering the Green's functions instead of the negative-energy solutions of the Klein-Gordon equation. The causality of the Green's functions (i.e. supp $S(x,.) \subset L_{x}$ ) simplifies the construction considerably. We could use it to avoid the explicit Fourier transformations of the proofs in [2]; furthermore, it makes the resummation of non-local contributions unnecessary. In this paper, the complications related to the non-causality of the negative-energy states will reappear in the light-cone expansion of the Dirac sea in Section 3. We point out that the light-cone expansion in [2] is more general in the way that the mass parameter $a=m^{2}$ need not be zero. This is sometimes more convenient, because then the mass is just a parameter of the Green's functions instead of occurring in the line integrals of the light-cone expansion. With our concept of dynamic mass matrices, however, an expansion around $a=0$ is more appropriate. The important generalization in 2.20) is that the two factors $S$ on the left side may be derivatives with respect to $a$. On the right side of equation (2.20), this is taken into account by the additional factors $\alpha^{l}(1-\alpha)^{r}$ in the integrand and by the higher order $(n+l+r+1)$ of the $a$-derivative of $S$. This generalization is the basis for the following iterations.

Lemma 2.1 can be used for the light-cone expansion of more complicated operator products. To explain the method, we look at the simplest example of three factors $S^{(0)}$ and two potentials $V, W$,

$$
\left(S^{(0)} V S^{(0)} W S^{(0)}\right)(x, y)=\int d^{4} z S^{(0)}(x, z) V(z)\left(S^{(0)} W S^{(0)}\right)(z, y) \quad .
$$

Having split up the operator product in this form, we can apply Lemma 2.1 to the factor $S^{(0)} W S^{(0)}$,

$$
=\sum_{n=0}^{\infty} \frac{1}{n !} \int d^{4} z S^{(0)}(x, z)\left\{V(z) \int_{0}^{1}\left(\alpha-\alpha^{2}\right)^{n}\left(\square^{n} W\right)_{\mid \alpha y+(1-\alpha) z} d \alpha\right\} S^{(n+1)}(z, y)
$$

Now we rewrite the $z$-integral as the operator product $\left(S^{(0)} g_{y} S^{(0)}\right)(x, y)$, where $g_{y}(z)$ is the function in the curly brackets. The $y$-dependence of $g_{y}$ causes no problems because we can view $y$ as a fixed parameter throughout the expansion. Thus we can simply apply Lemma 2.1 once again and obtain

$$
=\sum_{m, n=0}^{\infty} \frac{1}{m ! n !} \int_{0}^{1} d \beta(1-\beta)^{n+1}\left(\beta-\beta^{2}\right)^{m} \int_{0}^{1} d \alpha\left(\alpha-\alpha^{2}\right)^{n}
$$




$$
\times \square_{z}^{m}\left(V(z)\left(\square^{n} W\right)_{\mid \alpha y+(1-\alpha) z}\right)_{\mid z=\beta y+(1-\beta) x} S^{(m+n+2)}(x, y) \quad .
$$

The Laplacian $\square_{z}^{m}$ could be further computed with the Leibniz rule. Notice that the manipulations of the infinite sums are not problematic because the number of terms to every order on the light cone is actually finite (the situation would be more difficult if we studied the convergence of the sum (1.14), but, as pointed out earlier, the light-cone expansion is defined merely via the partial sums).

We want to iteratively perform the light-cone expansion of the operator products in (2.12). This is not directly possible with the method just described, because (2.12) contains the Dirac Green's function $s$ (instead of $S$ ). We must think about how to deal with this problem. Relation (2.11) allows us to replace the factors $s$ by $S$, but this gives additional partial derivatives in the operator product. These derivatives can be carried out after each iteration step using the Leibniz rule and the differentiation rule (2.17). In the simplest example, we have

$$
\begin{aligned}
& \left(s^{(0)} V S^{(0)}\right)(x, y)=\left(i \not \partial_{x}\right)\left(S^{(0)} V S^{(0)}\right)(x, y) \\
& =\quad i \not \partial_{x} \sum_{n=0}^{\infty} \frac{1}{n !} \int_{0}^{1}\left(\alpha-\alpha^{2}\right)^{n}\left(\square^{n} V\right)_{\mid \alpha y+(1-\alpha) x} S^{(n+1)}(x, y) \\
& =\quad i \sum_{n=0}^{\infty} \frac{1}{n !} \int_{0}^{1}(1-\alpha)\left(\alpha-\alpha^{2}\right)^{n}\left(\not \partial \square^{n} V\right)_{\mid \alpha y+(1-\alpha) x} S^{(n+1)}(x, y) \\
& \quad+\frac{i}{2} \sum_{n=0}^{\infty} \frac{1}{n !} \int_{0}^{1}\left(\alpha-\alpha^{2}\right)^{n}\left(\square^{n} V\right)_{\mid \alpha y+(1-\alpha) x}(y-x)_{j} \gamma^{j} S^{(n)}(x, y) .
\end{aligned}
$$

The only problem with this method is that the partial derivatives might hit a factor $S^{(0)}$, in which case rule (2.17) cannot be applied. In order to resolve this problem, we extend our constructions in a way which allows us to use all previous formulas also in this special case. For this, we take (2.17) as the defining equation for $(y-x)_{k} S^{(-1)}(x, y)$,

$$
(y-x)_{k} S^{(-1)}(x, y):=2 \frac{\partial}{\partial x^{k}} S^{(0)}(x, y)
$$

(notice that $S^{(-1)}$ itself remains undefined, only the combination $(y-x)_{k} S^{(-1)}(x, y)$ makes mathematical sense as the partial derivative of the distribution $S^{(0)}$ ). With this definition, the computation rule (2.18) becomes also valid for $l=-1$ : According to (2.18), the distribution $(y-x)^{2} S^{(0)}(x, y)$ vanishes identically, and thus

$$
\begin{aligned}
0 & =\square_{x}\left((y-x)^{2} S^{(0)}(x, y)\right) \\
& =\left(\square_{x}(y-x)^{2}\right) S^{(0)}(x, y)-4(y-x)^{j} \frac{\partial}{\partial x^{j}} S^{(0)}(x, y)+(y-x)^{2} \square_{x} S^{(0)}(x, y)
\end{aligned}
$$

In the last summand, we substitute (2.14), which gives $-(y-x)^{2} \delta^{4}(x-y)=0$. Using the identity $\square_{x}(y-x)^{2}=8$ and the definition (2.25), we conclude that

$$
(y-x)^{2} S^{(-1)}(x, y)=4 S^{(0)}(x, y) .
$$

The following lemma extends the result of Lemma 2.1 to the case when the right factor of the operator product is $S^{(-1)}$. 
Lemma 2.2 (light-cone expansion to first order for $r=-1$ ) The operator product $\left(S^{(l)} . S^{(-1)}\right), l \geq 0$, has the light-cone expansion

$$
\begin{gathered}
\int d^{4} z S^{(l)}(x, z) V(z)(y-z)_{k} S^{(-1)}(z, y)=\sum_{n=0}^{\infty} \frac{1}{n !} \int_{0}^{1} \alpha^{l}(1-\alpha)^{-1}\left(\alpha-\alpha^{2}\right)^{n} \\
\times \square_{z}^{n}\left(V(z)(y-z)_{k}\right)_{\mid z=\alpha y+(1-\alpha) x} d \alpha S^{(n+l)}(x, y) .
\end{gathered}
$$

Proof: We deduce the light-cone expansion for the left side of (2.27) from (2.20) by pulling one $y$-derivative outside,

$$
\begin{gathered}
\int d^{4} z S^{(l)}(x, z) V(z)(y-z)_{k} S^{(-1)}(z, y) \stackrel{(2.25), 2.19)}{=}-2 \frac{\partial}{\partial y^{k}}\left(S^{(l)} V S^{(0)}\right)(x, y) \\
=\quad-2 \frac{\partial}{\partial y^{k}} \sum_{n=0}^{\infty} \frac{1}{n !} \int_{0}^{1} \alpha^{l}\left(\alpha-\alpha^{2}\right)^{n}\left(\square^{n} V\right)_{\mid \alpha y+(1-\alpha) x} d \alpha S^{(n+l+1)}(x, y) \\
\sum_{n=0}^{\infty} \frac{1}{n !} \int_{0}^{1} \alpha^{l}\left(\alpha-\alpha^{2}\right)^{n}\left(\square^{n} V\right)_{\mid \alpha y+(1-\alpha) x} d \alpha(y-x)_{k} S^{(n+l)}(x, y) \\
-2 \sum_{n=0}^{\infty} \frac{1}{n !} \int_{0}^{1} \alpha^{l+1}\left(\alpha-\alpha^{2}\right)^{n}\left(\partial_{k} \square^{n} V\right)_{\mid \alpha y+(1-\alpha) x} d \alpha S^{(n+l+1)}(x, y)
\end{gathered}
$$

In (2.29), we substitute the identity

$$
\partial_{k} \square^{n} V(z)=-\frac{1}{2(n+1)} \square_{z}^{n+1}\left(V(z)(y-z)_{k}\right)+\frac{1}{2(n+1)}\left(\square_{z}^{n+1} V(z)\right)(y-z)_{k} .
$$

The contribution of the second summand in (2.30) cancels all the summands $n=1,2, \ldots$ of (2.28), and we get

$$
\begin{aligned}
& \int d^{4} z S^{(l)}(x, z) V(z)(y-z)_{k} S^{(-1)}(z, y)=\int_{0}^{1} \alpha^{l} V_{\mid \alpha y+(1-\alpha) x} d \alpha(y-x)_{k} S^{(l)}(x, y) \\
& +\sum_{n=0}^{\infty} \frac{1}{(n+1) !} \int_{0}^{1} \alpha^{l+1}\left(\alpha-\alpha^{2}\right)^{n} \square_{z}^{n+1}\left(V(z)(y-z)_{k}\right)_{\mid z=\alpha y+(1-\alpha) x} d \alpha S^{(n+1+l)}(x, y)
\end{aligned}
$$

After shifting the summation index, this coincides with (2.27).

Notice that the pole of the factor $(1-\alpha)^{-1}$ for $\alpha=1$ does not lead to problems in (2.27): In the case $n=0$, it disappears since $(1-\alpha)^{-1}(y-z)=y-x$; for $n>0$, it is compensated by the zero of the factor $\left(\alpha-\alpha^{2}\right)^{n}$.

After these preparations, we come to the light-cone expansion of general Feynman diagrams. For the line integrals of Lemma 2.1 and Lemma 2.2, we introduce the short notation

$$
\int_{x}^{y}[l, r \mid n] d z f(z):=\int_{0}^{1} d \alpha \alpha^{l}(1-\alpha)^{r}\left(\alpha-\alpha^{2}\right)^{n} f(\alpha y+(1-\alpha) x) .
$$

If $l, r$, and $n$ all vanish, we sometimes omit the bracket, i.e.

$$
\int_{x}^{y} d z f(z):=\int_{0}^{1} d \alpha f(\alpha y+(1-\alpha) x) .
$$

Furthermore, we abbreviate the following products with multi-indices

$$
\partial_{z}^{J}:=\frac{\partial}{\partial z^{j_{1}}} \cdots \frac{\partial}{\partial z^{j_{l}}} \quad, \quad(y-x)^{J}:=(y-x)^{j_{1}} \cdots(y-x)^{j_{l}} \quad, \quad \gamma^{J}:=\gamma^{j_{1}} \cdots \gamma^{j_{l}},
$$

where $J=\left(j_{1}, \ldots, j_{l}\right)$. 
Theorem 2.3 (inductive light-cone expansion) The light-cone expansion of the $k^{\text {th }}$ order contribution $\left((-s B)^{k} s\right)(x, y)$ to the perturbation series (2.19) can be written as an infinite sum of expressions, each of which has the form

$$
\begin{gathered}
\chi_{c} C(y-x)^{I} \int_{x}^{y}\left[l_{1}, r_{1} \mid n_{1}\right] d z_{1} \partial_{z_{1}}^{I_{1}} \square_{z_{1}}^{p_{1}} V_{J_{1}, c_{1}}^{(1)}\left(z_{1}\right) \int_{z_{1}}^{y}\left[l_{2}, r_{2} \mid n_{2}\right] d z_{2} \partial_{z_{2}}^{I_{2}} \square_{z_{2}}^{p_{2}} V_{J_{2}, c_{2}}^{(2)}\left(z_{2}\right) \\
\ldots \int_{z_{k-1}}^{y}\left[l_{k}, r_{k} \mid n_{k}\right] d z_{k} \partial_{z_{k}}^{I_{k}} \square_{z_{k}}^{p_{k}} V_{J_{k}, c_{k}}^{(k)}\left(z_{k}\right) \gamma^{J} S^{(h)}(x, y) .
\end{gathered}
$$

In this formula, $C$ is a complex number and the parameters $l_{a}, r_{a}, n_{a}$, and $p_{a}$ are nonnegative integers; the indices $c$ and $c_{a}$ can take the two values $L$ or $R$. The functions $V_{J_{a}, c_{a}}^{(a)}$ coincide with any of the individual potentials in (2.10) with chirality $c_{a}$, i.e.

$$
\begin{aligned}
V_{c_{a}}^{(a)} & =A_{c_{a}} & & \left(\text { in which case }\left|J_{a}\right|=1\right) \quad \text { or } \\
V_{c_{a}}^{(a)} & =m Y_{c_{a}} & & \text { (in which case } \left.\left|J_{a}\right|=0\right)
\end{aligned}
$$

The chirality $c_{a}$ of the potentials is determined by the following rules:

(i) The chirality $c_{1}$ of the first potential coincides with the chirality of the projector $\chi_{c}$.

(ii) The chirality of the potentials is reversed at every mass matrix, i.e.

$$
c_{a} \text { and } c_{a+1}\left\{\begin{array}{cl}
\text { coincide } & \text { if } V_{c_{a}}^{(a)}=A_{c_{a}} \\
\text { are opposite } & \text { if } V_{c_{a}}^{(a)}=m Y_{c_{a}}
\end{array} .\right.
$$

The tensor indices of the multi-indices in (2.39) are all contracted with each other, according to the following rules:

(a) No two tensor indices of the same multi-index are contracted with each other.

(b) The tensor indices of the factor $\gamma^{J}$ are all contracted with different multi-indices, in the order of their appearance in the product (2.39) (i.e., for $J=\left(j_{1}, \ldots, j_{l}\right)$ and $1 \leq a<b \leq l$, the multi-index with which $j_{a}$ is contracted must stand to the left of the multi-index corresponding to $j_{b}$ ).

The parameter $h$ is given by

$$
2 h=k-1-|I|+\sum_{a=1}^{k}\left(\left|I_{a}\right|+2 p_{a}\right) .
$$

The number of factors $(y-x)$ is bounded by

$$
|I| \leq k+1-\sum_{a=1}^{k}\left|I_{a}\right|
$$

Basically, this theorem states that the light-cone expansion of the $k^{\text {th }}$ order Feynman diagrams can be written with $k$ nested line integrals. Notice that, according to (1.7), the potentials $V^{(a)}\left(z_{a}\right)$ do in general not commute with each other, so that the order of multiplication is important in (2.32). In order to avoid misunderstandings, we point out that the derivatives $\partial_{z_{a}}^{I_{a}}$ and $\square_{z_{a}}^{p_{a}}$ do not only act on $V^{(a)}\left(z_{a}\right)$, but also on all the following factors $V^{(a+1)}\left(z_{a+1}\right), V^{(a+2)}\left(z_{a+2}\right), \ldots$ (note that the variables $z_{a+1}, z_{a+2}, \ldots$ 
implicitly depend on $z_{a}$ via the inductive definition of the line integrals). Clearly, these derivatives could be carried out further with the Leibniz rule, but it is easier not to do this at the moment. The restrictions (a) and (b) on the possible contractions of the tensor indices were imposed in order to avoid an abuse of our multi-index notation. More precisely, (a) prevents factors $(y-x)^{2}$ in $(y-x)^{I}$, an unnecessary large number of $\gamma$ matrices in $\gamma^{J}$, and "hidden" Laplacians in the partial derivatives $\partial_{z_{a}}^{I_{a}}$. The rule (b), on the other hand, prevents factors $(y-x)^{2}$ and hidden Laplacians in combinations of the form $(y-x)_{i}(y-x)_{j} \gamma^{i} \gamma^{j}$ and $\partial_{i j} V_{J_{a}}^{(a)} \gamma^{i} \gamma^{j}$, respectively. Our ordering condition for the $\gamma$-matrices is just a matter of convenience. Relation (2.34) is very useful because it immediately tells for any configuration of the line integrals and potentials in (2.32) what the corresponding order on the light cone is. Notice that (2.34) and (2.35) imply the inequality

$$
h \geq-1+\sum_{a=1}^{k}\left|I_{a}\right|+p_{a} .
$$

Especially, one sees that $h \geq-1$. In the case $h=-1$, (2.34) yields that $|I|>0$, so that (2.32) must contain at least one factor $(y-x)$. Therefore the factor $S^{(h)}$ in $(2.32)$ is always well-defined by either 2.13) or 2.25).

We point out that, although the total number of summands (2.32) is infinite, the number of summands for any given value of the parameter $h$ is finite. This is clear because, for fixed $h$, the relations (2.34) and (2.35) only allow a finite number of possibilities to choose the parameters $|I|,\left|I_{a}\right|$, and $p_{a}$; thus one only gets a finite combinatorics for all expressions of the form (2.32). Since, according to (2.6), the contributions for higher values of $h$ are of higher order on the light cone, we conclude that the number of summands (2.32) is finite to every order on the light cone. Therefore the light-cone expansion of Theorem 2.3 makes mathematical sense in terms of Def. 1.2.

Proof of Theorem 2.9: We proceed inductively in $k$. For $k=0$, the assumption is true because we can write the free Dirac Green's function in the required form (2.32),

$$
s(x, y) \stackrel{(2.11), 2.25)}{=}\left(\chi_{L}+\chi_{R}\right) \frac{i}{2}(y-x)^{j} \gamma_{j} S^{(-1)}(x, y)
$$

The conditions (i), (ii), (a), (b), and the relations (2.34), 2.35) are clearly satisfied.

Assume that the theorem holds for a given $k$. With the formula

$$
\left((-s B)^{k+1} s\right)(x, y) \stackrel{(2.11)}{=}-i \not \partial_{x} \int d^{4} z S^{(0)}(x, z) B(z)\left((-s B)^{k} s\right)(z, y)
$$

we can express the $(k+1)^{\text {st }}$ order contribution to the perturbation series (2.12) in terms of the $k^{\text {th }}$ order contribution. We must show that (2.38) can again be written as a sum of expressions of the form (2.32) (with $k$ replaced by $k+1$ ), and that (i), (ii), (a), (b), and (2.34), (2.35) are satisfied. This is done in several construction steps:

1) Chiral decomposition: We substitute the induction hypothesis (2.32) into (2.38). This gives a sum of expressions of the form

$$
\begin{gathered}
C i \not \phi_{x} \int d^{4} z S^{(0)}(x, z)\left\{(y-z)^{I} B(z) \chi_{c} \int_{z}^{y}\left[l_{1}, r_{1} \mid n_{1}\right] d z_{1} \partial_{z_{1}}^{I_{1}} \square^{p_{1}} V_{J_{1}, c_{1}}^{(1)}\left(z_{1}\right)\right. \\
\left.\cdots \int_{z_{k-1}}^{y}\left[l_{k}, r_{k} \mid n_{k}\right] d z_{k} \partial_{z_{k}}^{I_{k}} \square^{p_{k}} V_{J_{k}, c_{k}}^{(k)}\left(z_{k}\right) \gamma^{J}\right\} S^{(h)}(z, y)
\end{gathered}
$$


We insert the special form of the potential $B$, (2.10), and expand. Using the commutation rule $\gamma^{i} \chi_{L / R}=\chi_{R / L} \gamma^{i}$, we bring all chiral projectors to the very left, where they can be combined with the formula $\chi_{c} \chi_{d}=\delta_{c d} \chi_{c}$ to a single chiral projector. Next, we bring the $\gamma$-matrices of $B$ to the right and write them together with the factor $\gamma^{J}$ in (2.39) (notice that the Dirac matrices commute with the potentials $V_{c_{a}}^{(a)}$, which are only non-diagonal in the Dirac sea index). Denoting the individual potentials of the factor $B$ in (2.39) by $V_{J_{0}, c_{0}}^{(0)}$, we thus get for (2.39) a sum of expressions of the form

$$
\begin{gathered}
\chi_{c} C i \not \partial_{x} \int d^{4} z S^{(0)}(x, z)\left\{(y-z)^{I} V_{J_{0}, c_{0}}^{(0)}(z) \int_{z}^{y}\left[l_{1}, r_{1} \mid n_{1}\right] d z_{1} \partial_{z_{1}}^{I_{1}} \square^{p_{1}} V_{J_{1}, c_{1}}^{(1)}\left(z_{1}\right)\right. \\
\left.\cdots \int_{z_{k-1}}^{y}\left[l_{k}, r_{k} \mid n_{k}\right] d z_{k} \partial_{z_{k}}^{I_{k}} \square^{p_{k}} V_{J_{k}, c_{k}}^{(k)}\left(z_{k}\right) \gamma^{J}\right\} S^{(h)}(z, y)
\end{gathered}
$$

The chiral decomposition (2.10) and the induction hypothesis (i) yield that the chirality $c_{0}$ coincides with $c$, whereas $c_{1}$ coincides with $c_{0}$ if and only if $V_{c_{0}}^{(0)}=A_{c_{0}}$. Since the chiralities $c_{2}, c_{3}, \ldots$ satisfy the induction hypothesis (ii), we conclude that the rules (i) and (ii) are also satisfied in (2.40) (after relabeling the indices in an obvious way). The chirality of the potentials will not be affected in all the following construction steps; to simplify the notation, we will omit the indices $c_{a}$ from now on.

2) Light-cone expansion with Lemma 2.1, Lemma 2.9: Since y can be considered as a fixed parameter, we can in 2.40 apply either Lemma 2.1 or Lemma 2.2 with $V$ given by the expression in the curly brackets,

$$
\begin{aligned}
(2.40)= & \chi_{c} C i \not \partial_{x} \sum_{n=0}^{\infty} \frac{1}{n !} \int_{x}^{y}[0, h \mid n] d z \\
\times & \square_{z}^{n}\left((y-z)^{I} V_{J_{0}}^{(0)}(z) \int_{z}^{y}\left[l_{1}, r_{1} \mid n_{1}\right] d z_{1} \partial_{z_{1}}^{I_{1}} \square^{p_{1}} V_{J_{1}}^{(1)}\left(z_{1}\right)\right. \\
& \left.\cdots \int_{z_{k-1}}^{y}\left[l_{k}, r_{k} \mid n_{k}\right] d z_{k} \partial_{z_{k}}^{I_{k}} \square^{p_{k}} V_{J_{k}}^{(k)}\left(z_{k}\right)\right) \gamma^{J} S^{(n+h+1)}(x, y)
\end{aligned}
$$

3) Computation of the Laplacian $\square_{z}^{n}$ : We carry out the $z$-derivatives in (2.41) inductively with the Leibniz rule. Each derivative can act either on the factors $(y-z)^{I}$ or on the functions $V^{(a)}$. In the first case, one of the factors $(y-z)$ disappears. Thus we get a sum of expressions of the form

$$
\begin{gathered}
\chi_{c} C i \not \partial_{x} \int_{x}^{y}[0, h \mid n] d z(y-z)^{\hat{I}} \partial_{z}^{I_{0}} \square_{z}^{p_{0}} V_{J_{0}}^{(0)}(z) \int_{z}^{y}\left[l_{1}, r_{1} \mid n_{1}\right] d z_{1} \partial_{z_{1}}^{I_{1}} \square^{p_{1}} V_{J_{1}}^{(1)}\left(z_{1}\right) \\
\ldots \int_{z_{k-1}}^{y}\left[l_{k}, r_{k} \mid n_{k}\right] d z_{k} \partial_{z_{k}}^{I_{k}} \square^{p_{k}} V_{J_{k}}^{(k)}\left(z_{k}\right) \gamma^{J} S^{(n+h+1)}(x, y)
\end{gathered}
$$

with $|\hat{I}| \leq|I|$ and

$$
2 n=|I|-|\hat{I}|+\left|I_{0}\right|+2 p_{0} \quad .
$$

We can assume that no tensor indices of $\partial_{z}^{I_{0}}$ are contracted with each other (otherwise we rewrite the corresponding partial derivatives as additional Laplacians). Then all the partial derivatives $\partial_{z}$ in (2.42) were generated in the case when one derivative 
of a Laplacian $\square_{z}$ in (2.41) hit a factor $(y-z)$ whereas the other derivative acted on the $V^{(a)}$. Thus the number of factors $(y-z)$ which disappeared by carrying out the Laplacians in (2.41) is larger or equal than the number of partial derivatives $\partial_{z}$,

$$
|I|-|\hat{I}| \geq\left|I_{0}\right|
$$

4) Extraction of the factors $(y-x)$ : In (2.42), we iteratively apply the identity

$$
\int_{x}^{y}[0, r \mid n] d z(y-z) \cdots=(y-x) \int_{x}^{y}[0, r+1 \mid n] d z \cdots .
$$

This gives $(k+1)$ nested line integrals of the form

$$
\begin{gathered}
(2.42)=\chi_{c} C i \partial_{x}(y-x)^{\hat{I}} S^{(\hat{h})}(x, y) \int_{x}^{y}\left[l_{0}, r_{0} \mid n_{0}\right] d z_{0} \partial_{z_{0}}^{I_{0}} \square^{p_{0}} V_{J_{0}}^{(0)}\left(z_{0}\right) \\
\cdots \int_{z_{k-1}}^{y}\left[l_{k}, r_{k} \mid n_{k}\right] d z_{k} \partial_{z_{k}}^{I_{k}} \square^{p_{k}} V_{J_{k}}^{(k)}\left(z_{a}\right) \gamma^{J}
\end{gathered}
$$

with

$$
\begin{aligned}
& l_{0}=0, \quad r_{0}=h+|\hat{I}|, \quad n_{0}=n \\
& 0 \leq 2 \hat{h}=2(n+h+1) \stackrel{(2.43)}{=} 2 h+2+|I|-|\hat{I}|+\left|I_{0}\right|+2 p_{0} \quad .
\end{aligned}
$$

We can arrange that the parameters $l_{0}, r_{0}$, and $n_{0}$ are all positive: The only parameter which might be negative is $r_{0}$; in this case, $h=-1,|\hat{I}|=0$, and thus $r_{0}=-1$. The induction hypothesis (2.34) yields that $|I|>0$. Thus $|I|>|\hat{I}|$, and relation (2.43) gives that $\left(n_{0}=\right) n>0$. Therefore we can apply the identity

$$
\left[l_{0}, r_{0} \mid n_{0}\right]=\left[l_{0}+1, r_{0}+1 \mid n_{0}-1\right]
$$

to make all the parameters in this bracket positive.

5) Computation of the partial derivative $\not_{x}$ : The $x$-derivative in (2.46) can act on the factors $S^{(\hat{h})},(y-x)^{\hat{I}}$, or $V^{(a)}\left(z_{a}\right)$. The first case can be computed with the rules (2.17) or (2.25); it decreases $\hat{h}$ by one and gives one additional factor $(y-x)$. In the second case, one factor $(y-x)$ disappears, and thus $|\hat{I}|$ is decremented. The last case can be handled with the rule

$$
\frac{\partial}{\partial x^{k}} \int_{x}^{y}[l, r \mid n] d z f(z, y)=\int_{x}^{y}[l, r+1 \mid n] \frac{\partial}{\partial z^{k}} f(z, y),
$$

which increases $\left|I_{0}\right|$ by one. As is immediately verified in each of these cases, equation (2.48) transforms into

$$
2 \hat{h}=2 h+1+|I|-|\hat{I}|+\left|I_{0}\right|+2 p_{0},
$$

whereas inequality (2.44) must be weakened to

$$
|\hat{I}| \leq 1+|I|-\left|I_{0}\right|
$$

Finally, we combine the $\gamma$-matrix of the factor $\not_{x}$ with $\gamma^{J}$. 
After these transformations, the $(k+1)^{\text {st }}$ order Feynman diagram consists of a sum of terms of the form

$$
\begin{gathered}
\chi_{c} C(y-x)^{\hat{I}} \int_{x}^{y}\left[l_{0}, r_{0} \mid n_{0}\right] d z_{0} \partial_{z_{0}}^{I_{0}} \square_{z_{0}}^{p_{0}} V_{J_{0}}^{(0)}\left(z_{0}\right) \\
\ldots \int_{z_{k-1}}^{y}\left[l_{k}, r_{k} \mid n_{k}\right] d z_{k} \partial_{z_{k}}^{I_{k}} \square_{z_{k}}^{p_{k}} V_{J_{k}}^{(k)}\left(z_{k}\right) \gamma^{J} S^{(\hat{h})}(x, y)
\end{gathered}
$$

Notice that the parameters $I_{a}, p_{a}, a=1, \ldots, k$, were not changed by the above construction steps; they are still the same as in the induction hypothesis (2.32). After renaming the indices and the integration variables, (2.52) is of the required form (2.32). The conditions (a) and (b) for the contractions of the tensor indices, however, will in general be violated. Therefore we need two further computation steps:

6) Simplification of the $\gamma$-matrices: If any two of the tensor indices of the factor $\gamma^{J}$ are contracted with each other, we reorder the $\gamma$-matrices with the anti-commutation relations

$$
\left\{\gamma^{i}, \gamma^{j}\right\}=2 g^{i j} \mathbb{1}
$$

until the corresponding matrices are next to each other. Applying the identity $\gamma^{i} \gamma_{i}=4 \mathbb{1}$, both Dirac matrices disappear. We iterate this procedure until no tensor indices of $\gamma^{J}$ are contracted with each other (notice that the iteration comes to an end because the number of $\gamma$-factors is decreased by two in each step). Again using the anti-commutation rule (2.53), we reorder the Dirac matrices until they are in the same order in which the factors to which their tensor indices are contracted appear in the product (2.52). If any two of the $\gamma$-matrices are contracted with the same multi-index, these $\gamma$-matrices are next to each other, and we can use the symmetry in the tensor indices to eliminate them both, more precisely

$$
\begin{aligned}
(y-x)_{i}(y-x)_{j} \cdots \gamma^{i} \gamma^{j} & =(y-x)^{2} \cdots \mathbb{1} \\
\partial_{i j} V^{(a)} \cdots \gamma^{i} \gamma^{j} & =\square V^{(a)} \cdots \mathbb{1} .
\end{aligned}
$$

After all these transformations, condition (b) is satisfied.

Notice that the parameters $\left|I_{a}\right|$ and $p_{a}$ are in general changed in this construction step. More precisely, each transformation (2.55) modifies the parameters according to

$$
\left|I_{a}\right| \rightarrow\left|I_{a}\right|-2 \quad, \quad p_{a} \rightarrow p_{a}+1
$$

7) Handling of the new contractions: If any two tensor indices of a factor $\partial_{z_{a}}^{I_{a}}$ are contracted with each other, we rewrite the corresponding partial derivatives as a Laplacian; this changes the parameters $\left|I_{a}\right|$ and $p_{a}$ according to (2.56). If two tensor indices of the factor $(y-x)^{\hat{I}}$ are contracted with each other, this gives a factor $(y-x)^{2}$. Using the identity (2.18) or (2.26), we inductively absorb the factors $(y-x)^{2}$ into $S^{(\hat{h})}(x, y)$, which transforms $\hat{h}$ and $|\hat{I}|$ as

$$
\hat{h} \rightarrow \hat{h}+1, \quad|\hat{I}| \rightarrow|\hat{I}|-2 .
$$

After these transformations, condition (a) is also satisfied. 
After all these construction steps, the $(k+1)^{\text {th }}$ order Feynman diagram is a sum of terms of the form (2.52) satisfying the conditions $(a)$ and (b). It remains to show that the relations (2.34) and (2.35) remain valid in our inductive construction: As mentioned earlier, the parameters $I_{a}, p_{a}, a=1, \ldots, k$ are not changed in the construction steps 1) to 5). In the steps 6) and 7), the transformations (2.56) and (2.57) preserve both the induction hypothesis (2.34), 2.35) and the relations (2.50), (2.51), as is immediately verified. By substituting (2.50) and (2.51) into (2.56), (2.57), we conclude that

$$
2 \hat{h}=(k+1)-1-|\hat{I}|+\sum_{a=0}^{k}\left|I_{a}\right|+2 p_{a} \quad, \quad|\hat{I}| \leq(k+1)+1-\sum_{a=0}^{k}\left|I_{a}\right| .
$$

Note that this proof is constructive in the sense that it gives a procedure with which the light-cone expansion of every Feynman diagram can be carried out explicitly. The disadvantage of this procedure, however, is that the resulting formulas become more and more complicated to higher order perturbation theory. Therefore it is essential to rearrange and collect the contributions of all Feynman diagrams in a way which makes it clear how $\tilde{s}(x, y)$ looks like to every order on the light cone. In preparation for this analysis, which will be the task of the following Subsection 2.2, we shall now simplify the light-cone expansion of Theorem 2.3 a little bit. More precisely, we want to eliminate from (2.32) the partial derivatives in direction of the line integrals, i.e. those derivatives $\partial_{z_{l}}$ which are contracted with a factor $(y-x)$. We call these derivatives tangential. The following combinatorial lemma controls the number of tangential derivatives.

Lemma 2.4 The contributions (2.32) to the light-cone expansion of Theorem 2.3 satisfy for $a=1, \ldots, k$ the inequalities

$$
l_{a}+n_{a} \geq t_{a}-1 \quad \text { and } \quad r_{a}+n_{a} \geq \sum_{b=a}^{k} t_{b}
$$

where $t_{a}$ denotes the number of tensor indices of the multi-index $I_{a}$ which are contracted with the factor $(y-x)^{I}$.

Proof: As in the proof of Theorem 2.3, we proceed inductively in the order $k$ of the perturbation theory. For $k=0$, the inequalities (2.58) are trivially satisfied according to (2.37). Assume that (2.58) is true for a given $k$. We go through the construction steps 1) to 7) of Theorem 2.3 and check that the inequalities (2.58) then also hold in (2.52) for $a=0, \ldots, k$.

We first consider the case $a>0$. The parameters $l_{a}, r_{a}$, and $n_{a}$ remain unchanged in all the construction steps of Theorem 2.3. Furthermore, it is obvious that the parameters $t_{a}$ are not affected in the steps 1), 2), 4), and 7). In the steps 3) and 5), the computation of the derivatives $\square_{z}^{n}$ and $\not_{x}$ might annihilate some of the factors $(y-x)$ which were contracted with the factors $\partial_{z_{a}}^{I_{a}}$; this may decrease the parameters $t_{a}$. For the analysis of step 6), note that all $\gamma$-matrices which are contracted with factors $(y-x)$ stand to the left of those $\gamma$-matrices which are contracted with the $\partial_{z_{a}}^{I_{a}}, a=1, \ldots, k$ (this follows from the ordering condition (b) in the induction hypothesis and the fact that additional factors $(y-x)^{j} \cdots \gamma_{j}$ are only generated during the construction if the partial derivative $\not_{x}$ hits $S^{(\hat{h})}$ in step 5); in this case, the corresponding $\gamma$-matrix stands at the very left in $\gamma^{J}$ ). Therefore the commutations of the Dirac matrices do not lead to additional contractions between factors $(y-x)$ and $\partial_{z_{a}}^{I_{a}}$, which implies that the parameters $t_{a}$ remain unchanged 
in step 6). We conclude that the $l_{a}, r_{a}$, and $n_{a}$ remain unchanged whereas the $t_{a}$ may only decrease, and thus (2.58) holds for $a=1, \ldots, k$ throughout all the construction steps.

It remains to show that the inequalities (2.58) hold in (2.52) for $a=0$. We first look at the situation after step 4) in (2.46): The values (2.47) for $l_{0}, r_{0}$, and $n_{0}$ give in combination with (2.43) the equations

$$
\begin{aligned}
l_{0}+n_{0} & =\frac{1}{2}\left(|I|-|\hat{I}|+\left|I_{0}\right|+2 p_{0}\right) \\
r_{0}+n_{0} & =h+\frac{1}{2}\left(|I|+|\hat{I}|+\left|I_{0}\right|+2 p_{0}\right) .
\end{aligned}
$$

Moreover, the number of tangential derivatives $t_{0}$ at the first potential is clearly bounded by the total number of derivatives there,

$$
\left|I_{0}\right| \geq t_{0}
$$

Furthermore, the total number of tangential derivatives is smaller than the number of factors $(y-x)$,

$$
|\hat{I}| \geq \sum_{a=0}^{k} t_{a}
$$

Substituting (2.44) and (2.61) into (2.59) yields the inequalities

$$
l_{0}+n_{0} \geq\left|I_{0}\right|+p_{0} \geq t_{0} .
$$

In order to get a bound for $r_{0}+n_{0}$, we must distinguish two cases. If $h \geq 0$, we substitute (2.44) into 2.60) and get with (2.62) the inequality

$$
r_{0}+n_{0} \geq|\hat{I}|+\left|I_{0}\right|+p_{0} \geq|\hat{I}| \geq \sum_{a=0}^{k} t_{a} .
$$

In the case $h=-1,(2.36)$ shows that $\left|I_{a}\right|$, and consequently also $t_{a}$, vanish for $1 \leq a \leq k$. Furthermore, (2.34) yields that $|I| \neq 0$. Thus (2.60) and (2.61), (2.62) give the bound

$$
r_{0}+n_{0} \geq h+\frac{|I|}{2}+\frac{1}{2} \sum_{a=0}^{k} t_{a}+\frac{1}{2} t_{0} \geq \frac{1}{2} \sum_{a=0}^{k} t_{a}+\frac{1}{2} t_{0}
$$

where we used in the last inequality that $h+|I| / 2 \geq-1 / 2$ and that all the other terms are integers. Since $t_{0}=\sum_{a=0}^{k} t_{a}$, we conclude that inequality (2.64) also holds in the case $h=-1$.

We finally consider how the bounds (2.63) and (2.64) for $l_{0}+n_{0}$ and $r_{0}+n_{0}$ must be modified in the subsequent construction steps. In step 5), the partial derivative $\not_{x}$ may annihilate a factor $(y-x)$, in which case the parameters $t_{a}$ might decrease. On the other hand, the partial derivatives $\not_{x}$ may produce an additional factor $\partial_{z_{0}}$; in this case, $r_{0}$ is incremented according to (2.49). In step 6), only this additional factor $\partial_{z_{0}}$ may be contracted with $(y-x)^{\hat{I}}$. Step 7) does not change $l_{0}, r_{0}, n_{0}$, and $t_{0}$. Putting these transformations together, we conclude that the inequality (2.63) for $l_{0}+n_{0}$ must be weakened by one, whereas the bound (2.64) for $r_{0}+n_{0}$ remains valid as it is. This gives precisely the inequalities (2.58) for $a=0$. 
Theorem 2.5 (partial integration of the tangential derivatives) Every contribution (2.32) to the light cone expansion of Theorem 2.3 can be written as a finite sum of expressions of the form

$$
\begin{gathered}
\chi_{c} C(y-x)^{K} W^{(0)}(x) \int_{x}^{y}\left[l_{1}, r_{1} \mid n_{1}\right] d z_{1} W^{(1)}\left(z_{1}\right) \int_{z_{1}}^{y}\left[l_{2}, r_{2} \mid n_{2}\right] d z_{2} W^{(2)}\left(z_{2}\right) \\
\ldots \int_{z_{\alpha-1}}^{y}\left[l_{\alpha}, r_{\alpha} \mid n_{\alpha}\right] d z_{\alpha} W^{(\alpha)}\left(z_{\alpha}\right) \gamma^{J} S^{(h)}(x, y) \quad, \alpha \leq k
\end{gathered}
$$

where the factors $W^{(\beta)}$ are composed of the potentials and their partial derivatives,

$$
W^{(\beta)}=\left(\partial^{K_{a_{\beta}}} \square^{p_{a_{\beta}}} V_{J_{a_{\beta}}, c_{a_{\beta}}}^{\left(a_{\beta}\right)}\right) \cdots\left(\partial^{K_{b_{\beta}}} \square^{p_{b_{\beta}}} V_{J_{b_{\beta}}, c_{b_{\beta}}}^{\left(b_{\beta}\right)}\right)
$$

with $a_{1}=1, a_{\beta+1}=b_{\beta}+1, b_{\beta} \geq a_{\beta}-1$ (in the case $b_{\beta}=a_{\beta}-1, W^{(\beta)}$ is identically one), and $b_{\alpha}=k$. The parameters $l_{a}, r_{a}$, and $n_{a}$ are non-negative integers, $C$ is a complex number, and $c=L / R, c_{a}=L / R$ are chiral indices. The potentials $V^{(a)}$ are again given by (2.33); their chirality is determined by the rules (i) and (ii) of Theorem 2.3. The tensor indices of the multi-indices $J, K, J_{a}$, and $K_{a}$ are all contracted with each other, according to the rules (a),(b) of Theorem 2.3 and

(c) The tensor indices of $(y-x)^{K}$ are all contracted with the tensor indices of the factors $V_{J_{a}}^{(a)}$ or $\gamma^{J}$ (but not with the factors $\partial^{K_{a}}$ ).

We have the relation

$$
2 h=k-1-|K|+\sum_{a=1}^{k}\left(\left|K_{a}\right|+2 p_{a}\right) .
$$

Proof: The basic method for the proof is to iteratively eliminate those partial derivatives $\partial_{z_{a}}^{I_{a}}$ in $(2.32)$ which are contracted with a factor $(y-x)$. This is done with the partial integration formula

$$
\begin{aligned}
(y-x)^{j} \int_{x}^{y}[l, r \mid n] d z \partial_{j} f(z) \stackrel{(2.31)}{=} \int_{0}^{1} d \alpha \alpha^{l}(1-\alpha)^{r}\left(\alpha-\alpha^{2}\right)^{n} \frac{d}{d \alpha} f(\alpha y+(1-\alpha) x) \\
=\delta_{r+n, 0} f(y)-\delta_{l+n, 0} f(x) \\
\quad-(l+n) \int_{x}^{y}[l-1, r \mid n] d z f(z)+(r+n) \int_{x}^{y}[l, r-1 \mid n] d z f(z) .
\end{aligned}
$$

In order to see the main difficulty, consider the example of two nested line integrals with two tangential derivatives

$$
\begin{aligned}
(y-x)^{j}(y-x)^{k} \int_{x}^{y}[0,1 \mid 0] d z_{1} V^{(1)}\left(z_{1}\right) \int_{z_{1}}^{y}[0,1 \mid 0] d z_{2} \partial_{j k} V^{(2)}\left(z_{2}\right) \\
=(y-x)^{j} \int_{x}^{y}[0,0 \mid 0] d z_{1} V^{(1)}\left(z_{1}\right)\left(y-z_{1}\right)^{k} \int_{z_{1}}^{y}[0,1 \mid 0] d z_{2} \partial_{j k} V^{(2)}\left(z_{2}\right) \\
=-(y-x)^{j} \int_{x}^{y} d z_{1} V^{(1)}\left(z_{1}\right) \partial_{j} V^{(2)}\left(z_{1}\right) \\
\quad+(y-x)^{j} \int_{x}^{y} d z_{1} V^{(1)}\left(z_{1}\right) \int_{z_{1}}^{y} d z_{2} \partial_{j} V^{(2)}\left(z_{2}\right) .
\end{aligned}
$$

Although the line integrals in (2.69) satisfy the conditions of Theorem 2.3, the expression cannot be transformed into the required form (2.65). Namely in (2.70), we cannot eliminate 
the remaining tangential derivative (because partial integration would yield a term $(y-$ $\left.x)^{j} \partial_{j} V^{(1)}\left(z_{1}\right)\right)$. In (2.71), on the other hand, we can successfully perform a second partial integration

$$
\text { 2.71) }=\int_{x}^{y}[0,-1 \mid 0] d z_{1} V^{(1)}\left(z_{1}\right)\left(V^{(2)}(y)-V^{(2)}\left(z_{1}\right)\right),
$$

but then the second parameter in the bracket $[., . \mid$.$] becomes negative. More generally,$ we must ensure that the boundary terms contain no tangential derivatives, and that the parameters $l_{a}, r_{a}$, and $n_{a}$ stay positive in the construction.

Since the chirality of the potentials is not affected by the partial integrations, it is obvious that the rules (i) and (ii) of Theorem 2.3 will remain valid. For simplicity in notation, we will omit the indices $c_{a}$ in the following.

First of all, we split up the factor $(y-x)^{I}$ in $(\overline{2.32})$ in the form $(y-x)^{I}=(y-x)^{K}(y-$ $x)^{L}$, where $L$ are those tensor indices which are contracted with the partial derivatives $\partial_{z_{a}}^{I_{a}}, a=1, \ldots, k$. Setting $b=1$ and $z_{0}=x$, the first line integral in (2.32) can be written as

$$
\cdots\left(y-z_{b-1}\right)^{L} \int_{z_{b-1}}^{y}\left[l_{b}, r_{b} \mid n_{b}\right] d z_{b} \partial_{z_{b}}^{I_{b}} \square_{z_{b}}^{p_{b}} V_{J_{b}}^{(b)}\left(z_{b}\right) \cdots .
$$

We rewrite the tangential derivatives in this line integral as derivatives in the integration variable,

$$
=\cdots\left(y-z_{b-1}\right)^{N} \int_{0}^{1} d \alpha \alpha^{l}(1-\alpha)^{r}\left(\frac{d}{d \alpha}\right)^{q} \partial_{z_{b}}^{K_{b}} \square_{z_{b}}^{p_{b}} V_{J_{b}}^{(b)}\left(z_{b}\right) \cdots
$$

with $|L|=|N|+q$ and $l=l_{b}+n_{b}, r=r_{b}+n_{b}$. Lemma 2.4 gives the bounds

$$
l \geq q-1, \quad r \geq q+|N| .
$$

More generally, we use (2.73), (2.74) as our induction hypothesis, whereby the left factor '...' stands for all previous line integrals (which contain no tangential derivatives), and the right factor '...' stands for subsequent line integrals. The tensor indices of the factor $\left(y-z_{a-1}\right)^{N}$ must all be contracted with the partial derivatives $\partial_{z_{a}}^{I_{a}}$ for $a>b$ and thus give tangential derivatives in the subsequent line integrals. The induction step is to show that all the $\alpha$-derivatives in $(2.73)$ can be eliminated, and that we can write the resulting expressions again in the form (2.73), (2.74) with $b$ replaced by $b+1$. Under the assumption that this induction step holds, we can eliminate all tangential derivatives in $k$ steps. The resulting expressions are very similar to (2.65), (2.66). The only difference is that the derivatives $\partial^{K_{a}}$ and $\square^{p_{a}}$ in the resulting expressions are differential operators acting on all the following factors $V^{(a)}, V^{(a+1)}, \ldots$; in $(2.66)$, on the other hand, the partial derivatives act only on the adjacent potential $V^{(a)}$. In order to bring the resulting expressions into the required form, we finally carry out all the derivatives with the Leibniz rule and the chain rule 2.49).

For the proof of the induction step, we integrate in (2.73) $q$ times by parts (if $q$ is zero, we can skip the partial integrations; our expression is then of the form (2.76)). Since the powers of the factors $\alpha$ and $(1-\alpha)$ are decreased at most by one in each partial integration step, (2.74) implies that the boundary values vanish unless in the last step for $\alpha=0$. We thus obtain a sum of terms of the form

$$
\begin{aligned}
& \cdots\left(y-z_{b-1}\right)^{N} \partial_{z_{b}}^{K_{b}} \square_{z_{b}}^{p_{b}} V_{J_{b}}^{(b)}\left(z_{b}\right) \cdots{ }_{z_{b} \equiv z_{b-1}} \quad \text { and } \\
& \cdots\left(y-z_{b-1}\right)^{N} \int_{z_{b-1}}^{y}[l, r \mid n=0] d z_{b} \partial_{z_{b}}^{K_{b}} \square_{z_{b}}^{p_{b}} V_{J_{b}}^{(b)}\left(z_{b}\right) \cdots \quad \text { with } l \geq 0, r \geq|N| .
\end{aligned}
$$


In (2.76), we iteratively use the relation

$$
(y-x)^{j} \int_{x}^{y}[l, r \mid n] d z \cdots=\int_{x}^{y}[l, r-1 \mid n] d z(y-z)^{j} \cdots
$$

to bring all factors $\left(y-z_{b-1}\right)$ to the right,

$$
(2.76)=\cdots \int_{z_{b-1}}^{y}[l, r \mid n=0] d z_{b}\left(y-z_{b}\right)^{N} \partial_{z_{b}}^{K_{b}} \square_{z_{b}}^{p_{b}} V_{J_{b}}^{(b)}\left(z_{b}\right) \cdots \quad \text { with } l, r \geq 0 .
$$

It might be convenient to reorganize the polynomials in this line integral with the identity

$$
\int_{x}^{y}[l, r \mid n] d z \cdots=\int_{x}^{y}[l-1, r-1 \mid n+1] d z \cdots,
$$

but this is not relevant for the statement of the theorem.

In both cases (2.75) and (2.78), we have an expression of the form

$$
\cdots\left(y-z_{b}\right)^{N} \partial_{z_{b}}^{K_{b}} \square_{z_{b}}^{p_{b}} V_{J_{b}}^{(b)}\left(z_{b}\right) \cdots
$$

where the first factor '...' stands for line integrals without tangential derivatives, and where none of the factors $\left(y-z_{b}\right)$ are contracted with $\partial_{z_{b}}^{K_{b}}$. Applying the "inverse Leibniz rules"

$$
\begin{aligned}
(y-x)^{j} \frac{\partial}{\partial x^{k}} & =\frac{\partial}{\partial x^{k}}(y-x)^{j}+\delta_{k}^{j} \\
(y-x)_{j} \square_{x} & =\square_{x}(y-x)_{j}+2 \frac{\partial}{\partial x^{j}},
\end{aligned}
$$

we iteratively commute all factors $\left(y-z_{b}\right)$ in 2.79 to the right. This gives a sum of expressions of the form

$$
\cdots \partial_{z_{b}}^{K_{b}} \square_{z_{b}}^{p_{b}} V_{J_{b}}^{(b)}\left(z_{b}\right)\left(y-z_{b}\right)^{L} \cdots
$$

where the factors $\left(y-z_{b}\right)$ are all contracted with the partial derivatives $\partial_{z_{a}}^{I_{a}}, a=b+1, \ldots, k$. The Leibniz rules may have annihilated some factors $\left(y-z_{b}\right)$ (i.e., $|L|$ might be smaller than $|N|)$; in this case, the parameters $t_{a}, a=b+1, \ldots, k$ have decreased. As a consequence, the inequalities of Lemma 2.4 are still valid for all expressions (2.82). If we write (2.82) in the form (2.72) with $b$ replaced by $b+1$, we can thus split up the tangential derivatives in the form (2.73),(2.74), which concludes the proof of the induction step.

It remains to derive equation (2.67): Note that each partial integration decreases both the number of factors $\left(y-z_{a-1}\right)$ and the total number of partial derivatives by one. If we carry out the remaining derivatives with the Leibniz rule (in the last step of the proof), this does not change the total order $\sum_{a=1}^{k}\left|K_{a}\right|+2 p_{a}$ of the derivatives. Therefore relation (2.34) in Theorem 2.3 transforms into (2.67).

\subsection{Resummation, Reduction to the Phase-Free Contribution}

In the previous subsection, we gave a procedure for performing the light-cone expansion of every summand of the perturbation series (2.12). In order to obtain formulas for the lightcone expansion of $\tilde{s}$, we must sum up the light-cone expansions of all Feynman diagrams. 
Collecting the contributions of all Feynman diagrams gives, to every order on the light cone, an infinite number of terms. To get control over all these terms, we shall reorder the sums and partly carry them out. In the end, the light-cone expansion for $\tilde{s}(x, y)$ will, to any order on the light cone, consist of only a finite number of summands. This rearrangement and simplification of the sums is called resummation of the light-cone expansion.

In order to get a first impression of what needs to be done, we consider the leading singularity on the light cone (more precisely, we neglect all terms of the order $\mathcal{O}((y-$ $\left.x)^{-2}\right)$ ). This corresponds to taking only the contributions with $h=-1$ in Theorem 2.3. According to the bound (2.36), the multi-indices $I_{a}$ and the parameters $p_{a}$ must all vanish. Furthermore, equation (2.34) yields that $|I|=k+1$. The only possibility to satisfy the rules $(a)$ and $(b)$ is to contract one factor $(y-x)$ with each potential $V^{(a)}, a=1, \ldots, k$, and the remaining factor $(y-x)$ with a $\gamma$-matrix. Thus the potentials $V^{(a)}$ must all be chiral potentials $A_{L / R}$. According to the rules (a) and (b), the chirality of all potentials must coincide with the chirality of the projector $\chi_{c}$. We conclude that the leading order of $\left((-s B)^{k} s\right)(x, y)$ on the light cone consists of a sum of expressions of the form

$$
\begin{aligned}
\chi_{c} C(y-x)_{j_{1}} \cdots(y-x)_{j_{k}} \int_{x}^{y}\left[l_{1}, r_{1} \mid n_{1}\right] d z_{1} A_{c}^{j_{1}}\left(z_{1}\right) \\
\quad \ldots \int_{z_{k-1}}^{y}\left[l_{k}, r_{k} \mid n_{k}\right] d z_{k} A_{c}^{j_{k}}\left(z_{k}\right)(y-x)^{j} \gamma_{j} S^{(-1)}(x, y)
\end{aligned}
$$

with $c=L$ or $c=R$. Thus Theorem 2.3 makes a precise statement on the mathematical structure of all the contributions to the light-cone expansion. However, it does not give information about the values of the parameters $C, l_{a}, r_{a}$, and $n_{a}$. In order to see more precisely how the leading order on the light cone looks like, we perform the light-cone expansion directly with Lemma 2.2. To first order in the external potential, we obtain

$$
\begin{aligned}
& (-s B s)(x, y) \stackrel{(2.11),(2.25)}{=} \frac{1}{2} \not \partial_{x} \int d^{4} z S^{(0)}(x, z)\left(B(z)(y-z)^{k} \gamma_{k}\right) S^{(-1)}(z, y)
\end{aligned}
$$

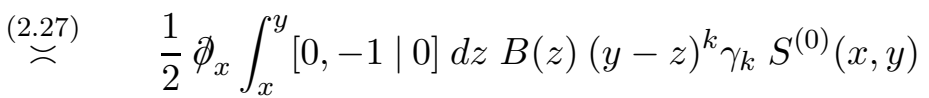

$$
\begin{aligned}
& \stackrel{2.25}{\asymp} \frac{1}{4} \int_{x}^{y} d z(y-x)^{j} \gamma_{j} B(z)(y-x)^{k} \gamma_{k} S^{(-1)}(x, y) \\
& \asymp \quad \frac{1}{2} \int_{x}^{y} d z\left(\chi_{L} A_{L}^{j}(z)+\chi_{R} A_{R}^{j}(z)\right)(y-x)_{j}(y-x)^{k} \gamma_{k} S^{(-1)}(x, y) \\
& \text { 2.25,2.11 }-i(y-x)_{j} \int_{x}^{y} d z\left(\chi_{L} A_{L}^{j}(z)+\chi_{R} A_{R}^{j}(z)\right) s(x, y) \text {, }
\end{aligned}
$$

where ' $\asymp$ ' denotes that we only take the leading order on the light cone. Since (2.83) is a product of a smooth function with a single factor $s(x, y)$, this formula can be immediately iterated. We obtain for the left and right handed component of the $k^{\text {th }}$ order Feynman diagram

$$
\begin{gathered}
\chi_{c}\left((-s B)^{k} s\right)(x, y) \asymp \chi_{c}(-i)^{k}(y-x)_{j_{1}} \cdots(y-x)_{j_{k}} \int_{x}^{y}[0, k-1 \mid 0] d z_{1} A_{c}^{j_{1}}\left(z_{1}\right) \\
\quad \times \int_{z_{1}}^{y}[0, k-2 \mid 0] d z_{2} A_{c}^{j_{2}}\left(z_{2}\right) \cdots \int_{z_{k-1}}^{y}[0,0 \mid 0] d z_{k} A_{c}^{j_{k}}\left(z_{k}\right) s(x, y) .
\end{gathered}
$$

(Notice that, according to Lemma 2.1, the higher order terms which were neglected in (2.83) also give contributions of the order $\mathcal{O}\left((y-x)^{-2}\right)$ in the iteration. Therefore it is 
really sufficient to take only the leading contribution on the light cone in every step.) The line integrals in (2.84) are particularly simple. Namely, they are the $k^{\text {th }}$ order contributions to the familiar Dyson series. From this we conclude that, to leading order on the light cone, the sum over all Feynman diagrams converges absolutely. We can carry out the sum and obtain

$$
\chi_{c} \tilde{s}(x, y) \asymp \chi_{c} \operatorname{Pexp}\left(-i \int_{0}^{1} A_{c \mid \alpha y+(1-\alpha) x}^{j}(y-x)_{j} d \alpha\right) s(x, y)
$$

where Pexp is the usual ordered exponential. For completeness, we give its definition:

Def. 2.6 For a smooth one-parameter family of matrices $F(\alpha), \alpha \in \mathbb{R}$, the ordered exponential $\operatorname{Pexp}\left(\int F(\alpha) d \alpha\right)$ is given by the Dyson series

$$
\begin{aligned}
\operatorname{Pexp}\left(\int_{a}^{b} F(\alpha) d \alpha\right)= & \mathbb{1}+\int_{a}^{b} d t_{0} F\left(t_{0}\right) d t_{0}+\int_{a}^{b} d t_{0} F\left(t_{0}\right) \int_{t_{0}}^{b} d t_{1} F\left(t_{1}\right) \\
& +\int_{a}^{b} d t_{0} F\left(t_{0}\right) \int_{t_{0}}^{b} d t_{1} F\left(t_{1}\right) \int_{t_{1}}^{b} d t_{2} F\left(t_{2}\right)+\cdots
\end{aligned}
$$

As is verified by a direct calculation, the ordered exponential is a solution of the ordinary differential equation

$$
\frac{d}{d a} \operatorname{Pexp}\left(\int_{a}^{b} F(\alpha) d \alpha\right)=-F(a) \operatorname{Pexp}\left(\int_{a}^{b} F(\alpha) d \alpha\right)
$$

with the boundary conditions

$$
\operatorname{Pexp}\left(\int_{b}^{b} F(\alpha) d \alpha\right)=\mathbb{1} .
$$

Because of the uniqueness of the solutions of ordinary differential equations, one can alternatively take (2.86), (2.87) as the definition for the ordered exponential.

For the ordered exponential in (2.85), we also use the shorter notations

$$
\operatorname{Pexp}\left(-i \int_{x}^{y} A_{c}^{j}(y-x)_{j}\right) \quad \text { and } \quad \mathrm{Pe}^{-i \int_{x}^{y} A_{c}^{j}(y-x)_{j}} .
$$

Notice that (2.88) is a unitary $(f \times f)$-matrix which depends only on the chiral potentials along the line segment $\overline{x y}$. Its inverse is

$$
\operatorname{Pexp}\left(-i \int_{x}^{y} A_{c}^{j}(y-x)_{j}\right)^{\dagger}=\operatorname{Pexp}\left(-i \int_{y}^{x} A_{c}^{j}(x-y)_{j}\right) .
$$

If the chiral potentials are commutative, i.e. $\left[A_{c}(x), A_{c}(y)\right]=0$, then the ordered exponential coincides with the ordinary exponential (this is e.g. the case if one considers the system $f=1$ of only one Dirac sea). For the ordered exponential (2.88) along a line

${ }^{1}$ We mention for clarity that this is in general not the same as

$$
\operatorname{Pexp}\left(i \int_{x}^{y} A_{c}^{j}(y-x)_{j}\right),
$$

because the exponentials in (2.90) and 2.89 are ordered in opposite directions. 
segment in Minkowski space, the differential equation (2.86) can be written with partial derivatives as

$$
(y-x)^{k} \frac{\partial}{\partial x^{k}} \mathrm{Pe}^{-i \int_{x}^{y} A_{c}^{j}(y-x)_{j}}=i(y-x)_{k} A_{c}^{k}(x) \mathrm{Pe}^{-i \int_{x}^{y} A_{c}^{j}(y-x)_{j}} .
$$

We conclude that, to leading order on the light cone, the special form of the formulas of the light-cone expansion allows us to immediately carry out the sum over all Feynman diagrams. Unfortunately, the situation to higher order on the light cone is more difficult, because the combinatorics of the partial derivatives and of the tensor contractions becomes very complicated. We cannot expect that the sum over all Feynman diagrams can then still be written in a simple, closed form. Nevertheless, ordered exponentials over the chiral potentials should be helpful. More precisely, it is promising to write the light-cone expansion with line integrals which contain intermediate ordered exponentials, like e.g. the line integral

$$
\int_{x}^{y} d z \mathrm{Pe}^{-i \int_{x}^{z} A_{L}^{j}(z-x)_{j}}\left(\square A_{L}(z)\right) \mathrm{Pe}^{-i \int_{z}^{y} A_{L}^{k}(y-z)_{k}}
$$

(expressions of this form are also suggested in view of the behavior of the fermionic projector under local gauge transformations of the external potential). Our basic idea is to arrange the contributions to the light-cone expansion of Theorem 2.5 to any given order on the light cone in such a way that all infinite sums (which arise from the fact that we have an infinite number of Feynman diagrams) can be carried out giving ordered exponentials. We want to end up with a finite number of terms which are of the form (2.65) with the only exception that the nested line integrals contain, similar to (2.92), additional ordered exponentials.

Before we can make this idea mathematically precise, we must clarify the connection between the line integrals in (2.65) and the line integrals with intermediate ordered exponentials. For this, we consider the example (2.92). If we expand the ordered exponentials in a Dyson series and reparametrize the integrals, (2.92) goes over into an infinite sum of nested line integrals of the form as in (2.65), more precisely

$$
\begin{gathered}
(2.92)=\sum_{p, q=0}^{\infty} \int_{x}^{y}\left[l_{1}, r_{1} \mid n_{1}\right] d z_{1} A_{L}^{j_{1}}\left(z_{1}\right)(y-x)_{j_{1}} \int_{z_{1}}^{y}\left[l_{2}, r_{2} \mid n_{2}\right] d z_{2} A_{L}^{j_{2}}\left(z_{2}\right)(y-x)_{j_{2}} \\
\quad \ldots \int_{z_{p-1}}^{y}\left[l_{p}, r_{p} \mid n_{p}\right] d z_{p} A_{L}^{j_{p}}\left(z_{p}\right)(y-x)_{j_{p}} \int_{z_{p}}^{y}[l, r \mid n] d z \square A_{L}(z) \\
\times \int_{z}^{y}\left[l_{p+1}, r_{p+1} \mid n_{p+1}\right] d z_{p+1} A_{L}^{j_{p+1}}\left(z_{p+1}\right)(y-x)_{j_{p+1}} \int_{z_{p+1}}^{y} A_{L}^{j_{p+2}}\left(z_{p+2}\right)(y-x)_{j_{p+2}} \\
\quad \ldots \int_{z_{p+q-1}}^{y}\left[l_{p+1}, r_{p+q} \mid n_{p+1}\right] d z_{p+q} A_{L}^{j_{p+q}}\left(z_{p+q}\right)(y-x)_{j_{p+q}} .
\end{gathered}
$$

The formulas of our desired light-cone expansion must be such that, after expanding the ordered exponentials in this way, we get precisely all the contributions to the light-cone expansion of Theorem 2.5. We point out that we can view the expansion (2.93) as a power series in the functions $A_{L / R}^{j}(y-x)_{j}$. The leading contribution to this power series is simply the line integral

$$
\int_{x}^{y} d z \square A_{L}(z) ;
$$

it is obtained from (2.92) by taking out the ordered exponentials there. In view of this example, we can hope to get the contributions to our desired expansion without the ordered 
exponentials (i.e. with all the ordered exponentials removed from the formulas) by picking those contributions to the light-cone expansion of Theorem 2.5 which contain no factors $A_{L / R}^{j}(y-x)_{j}$. We take these contributions as the starting point for our construction.

Def. 2.7 A contribution (2.65), 2.60) to the light-cone expansion of Theorem 2.5 is called phase-free if all the tangential potentials $V_{J_{a}}^{(a)}$ are differentiated, i.e.

$$
\left|K_{a}\right|+2 p_{a}>0 \quad \text { whenever } \quad J_{a} \text { is contracted with }(y-x)^{K} .
$$

To leading order on the light cone, the only phase-free contribution is the free Green's function $s$ (namely, according to (2.84), the contributions with $k \geq 1$ all contain factors $\left.A_{L / R}^{j}(y-x)_{j}\right)$. The restriction to the phase-free contribution also simplifies the situation in the general case. Namely, the following proposition shows that the phase-free contributions of the higher order Feynman diagrams involve higher mass-derivatives of the Green's functions.

Proposition 2.8 For every phase-free contribution (2.65) to the light-cone expansion of the $k^{\text {th }}$ order Feynman diagram $\left((-s B)^{k} s\right)(x, y)$, the parameter $h$ satisfies the bound

$$
h \geq-1+\left[\frac{k+1}{2}\right],
$$

where [.] denotes the Gauß bracket.

Proof: Consider a phase-free contribution (2.65) of the $k^{\text {th }}$ order Feynman diagram. According to the rules for the possible contractions of the tensor indices, only one factor $(y-x)$ may be contracted with $\gamma^{J}$; the remaining $|K|-1$ factors $(y-x)$ must be contracted with the $V_{J_{a}, c_{a}}^{(a)}$. Thus at least $|K|-1$ potentials are tangential and must (according to Def. 2.7) be differentiated. This gives the inequality

$$
|K|-1 \leq \sum_{a=1}^{k}\left(\left|K_{a}\right|+2 p_{a}\right) .
$$

We substitute this bound into (2.67) and obtain $2 h \geq-2+k$; this is equivalent to (2.94).

According to the explicit formula (2.5), the higher mass-derivatives of the Green's functions are of higher order on the light cone. More precisely, (2.94) and (2.6) yield that the phasefree contribution to the light-cone expansion of the $k^{\text {th }}$ order Feynman diagram is of the order

$$
\mathcal{O}\left((y-x)^{2 g}\right) \quad \text { with } \quad g=-2+\left[\frac{k+1}{2}\right] .
$$

This means that, to every order on the light cone, only a finite number of Feynman diagrams contribute. As a consequence, there are, to every order on the light cone, only a finite number of phase-free terms.

The phase-free contributions are useful because our desired light-cone expansion is obtained from them by inserting ordered exponentials into the line integrals. We do this "by hand," according to simple rules.

Def. 2.9 For every phase-free contribution (2.65) to the light-cone expansion of Theorem 2.5, we introduce a corresponding phase-inserted contribution. It is constructed according to the following rules: 
(I) We insert one ordered exponential into each line integral and one ordered exponential at the very end. More precisely, the phase-inserted contribution has the form

$$
\begin{gathered}
\chi_{c} C(y-x)^{K} W^{(0)}(x) \int_{x}^{y}\left[l_{1}, r_{1} \mid n_{1}\right] d z_{1} \mathrm{Pe}^{-i \int_{x}^{z_{1}} A_{c_{1}}^{j_{1}}\left(z_{1}-x\right)_{j_{1}}} W^{(1)}\left(z_{1}\right) \\
\times \int_{z_{1}}^{y}\left[l_{2}, r_{2} \mid n_{2}\right] d z_{2} \mathrm{Pe}^{-i \int_{z_{1}}^{z_{2}} A_{c_{2}}^{j_{2}}\left(z_{2}-z_{1}\right)_{j_{2}}} W^{(2)}\left(z_{2}\right) \cdots \\
\times \int_{z_{\alpha-1}}^{y}\left[l_{\alpha}, r_{\alpha} \mid n_{\alpha}\right] d z_{\alpha} \mathrm{Pe}^{-i \int_{z_{\alpha-1}}^{z_{\alpha}} A_{c_{\alpha}}^{j_{\alpha}}\left(z_{\alpha}-z_{\alpha-1}\right)_{j_{1}}} W^{(\alpha)}\left(z_{\alpha}\right) \\
\times \mathrm{Pe}^{-i \int_{z_{\alpha}}^{y} A_{c_{\alpha+1}}^{j_{\alpha+1}}\left(y-z_{\alpha}\right)_{j_{\alpha+1}}} \gamma^{J} S^{(h)}(x, y)
\end{gathered}
$$

(II) The chirality $c_{\beta}, \beta=1, \ldots, \alpha+1$ of the potentials in the ordered exponentials is determined by the number of dynamic mass matrices in the factors $W^{(\beta)}$; namely

$$
c_{\beta-1} \text { and } c_{\beta}\left\{\begin{array}{c}
\text { coincide } \\
\text { are opposite }
\end{array}\right\} \text { if } W^{(\beta-1)} \text { contains an }\left\{\begin{array}{c}
\text { even } \\
\text { odd }
\end{array}\right\} \text { number of factors } Y \text {, }
$$

where $c_{0}:=c$ is the chirality of the projector $\chi_{c}$ in (2.96).

To illustrate these insertion rules, we consider the example of two nested line integrals

$$
\chi_{L} \int_{x}^{y} d z_{1}\left(\square A_{L}\right)\left(z_{1}\right) \int_{z_{1}}^{y} d z_{2} m Y_{L}\left(z_{2}\right) S^{(1)}(x, y) .
$$

The corresponding phase-inserted contribution is

$$
\begin{aligned}
& \chi_{L} \int_{x}^{y} d z_{1} \mathrm{Pe}^{-i \int_{x}^{z_{1}} A_{L}^{j}\left(z_{1}-x\right)_{j}}\left(\square A_{L}\right)\left(z_{1}\right) \\
& \quad \times \int_{z_{1}}^{y} d z_{2} \mathrm{Pe}^{-i \int_{z_{1}}^{z_{2}} A_{L}^{k}\left(z_{2}-z_{1}\right)_{k}} m Y_{L}\left(z_{2}\right) \mathrm{Pe}^{-i \int_{z_{2}}^{y} A_{R}^{l}\left(y-z_{2}\right)_{l}} S^{(1)}(x, y) .
\end{aligned}
$$

Theorem 2.10 The light-cone expansion of the Green's function $\tilde{s}(x, y)$ coincides with the sum of all phase-inserted contributions.

Proof: A possible method for the proof would be to rearrange all the contributions to the light-cone expansion of Theorem 2.5 until recovering the Dyson series of the ordered exponentials in (2.96). However, this method has the disadvantage of being technically complicated. It is more elegant to use a particular form of "local gauge invariance" of the Green's function for the proof: First we will, for given $x$ and $y$, locally transform the spinors. The transformation will be such that the light-cone expansion for the transformed Green's function $\hat{s}(x, y)$ consists precisely of all phase-free contributions. Using the transformation law of the Green's function, we then show that the light-cone expansion of $\tilde{s}(x, y)$ is obtained from that of $\hat{s}(x, y)$ by inserting unitary matrices into the line integrals. Finally, we prove that these unitary matrices coincide with the ordered exponentials in Def. 2.9.

In preparation, we consider the transformation law of the Dirac operator and the Green's function under generalized local phase transformations of the spinors: We let $U_{L}$ and $U_{R}$ be two unitary matrix fields acting on the Dirac sea index,

$$
U_{L}=\left(U_{L}(x)_{m}^{l}\right)_{l, m=1, \ldots, f}, \quad U_{R}=\left(U_{R}(x)_{m}^{l}\right)_{l, m=1, \ldots, f} \quad \text { with } \quad U_{L} U_{L}^{*}=\mathbb{1}=U_{R} U_{R}^{*},
$$


and transform the wave functions $\Psi=\left(\Psi_{l}^{\alpha}(x)\right)_{l=1, \ldots, f}^{\alpha=1, \ldots, 4}$ according to

$$
\Psi(x) \rightarrow \hat{\Psi}(x)=U(x) \Psi(x) \quad \text { with } \quad U(x)=\chi_{L} U_{L}(x)+\chi_{R} U_{R}(x) .
$$

Thus $U_{L}$ and $U_{R}$ independently transform the left and right handed component of the wave functions, respectively. Notice that the transformation $U$ is not unitary with respect to our scalar product (1.4), because

$$
\begin{aligned}
V & :=U^{-1}=\chi_{L} U_{L}^{-1}+\chi_{R} U_{R}^{-1} \quad \text { but } \\
U^{*} & =\gamma^{0} U^{\dagger} \gamma^{0}=\chi_{R} U_{L}^{-1}+\chi_{L} U_{R}^{-1} .
\end{aligned}
$$

Therefore we must in the following carefully distinguish between $U, U^{*}$ and their inverses $V, V^{*}$. As an immediate consequence of the Dirac equation $(i \not \partial+\mathcal{B}-m) \Psi=0$, the transformed wave functions $\hat{\Psi}$ satisfy the equation

$$
V^{*}(i \not \partial+B) V \hat{\Psi}=0 \text {. }
$$

A short computation yields for the transformed Dirac operator

$$
V^{*}(i \not \partial+B) V=i \not \partial+\hat{B}
$$

with

$$
\hat{B}=\chi_{L}\left(\hat{A}_{R}-m \hat{Y}_{R}\right)+\chi_{R}\left(\hat{A}_{L}-m \hat{Y}_{L}\right),
$$

whereby $\hat{A}_{L / R}$ and $\hat{Y}_{L / R}$ are the potentials

$$
\begin{aligned}
& \hat{A}_{L / R}^{j}=U_{L / R} A_{L / R}^{j} U_{L / R}^{-1}+i U_{L / R}\left(\partial^{j} U_{L / R}^{-1}\right) \\
& \hat{Y}_{L / R}=U_{L / R} Y_{L / R} U_{R / L}^{-1} .
\end{aligned}
$$

We denote the advanced and retarded Green's functions of the transformed Dirac operator $i \not \partial+\hat{B}$ by $\hat{s}$. They satisfy the equation

$$
\left(i \not \partial_{x}+\hat{B}(x)\right) \hat{s}(x, y)=\delta^{4}(x-y) .
$$

Since we can view $\hat{B}$ as the perturbation of the Dirac operator, the Green's function $\hat{s}$ is, in analogy to (2.12), given by the perturbation series

$$
\hat{s}=\sum_{n=0}^{\infty}(-s \hat{B})^{n} s
$$

The important point for the following is that the Green's functions $\tilde{s}$ and $\hat{s}$ are related to each other by the local transformation

$$
\hat{s}(x, y)=U(x) \tilde{s}(x, y) U(y)^{*} .
$$

This is verified as follows: The right side of (2.102) also satisfies the defining equation (2.100) of the Green's functions; namely

$$
\begin{aligned}
& \left(i \not \partial_{x}+\hat{B}(x)\right) U(x) \tilde{s}(x, y) U(y)^{*}=V(x)^{*}\left(i \not \partial_{x}+B(x)\right) V(x) U(x) \tilde{s}(x, y) U(y)^{*} \\
& \quad=V(x)^{*}\left(i \not \partial_{x}+B(x)\right) \tilde{s}(x, y) U(y)^{*}=V(x)^{*} \delta^{4}(x-y) U(y)^{*} \\
& \quad=V(x)^{*} U(x)^{*} \delta^{4}(x-y)=\delta^{4}(x-y) .
\end{aligned}
$$


Furthermore, the supports of both sides of (2.102) lie (depending on whether we consider the advanced or retarded Green's functions) either in the upper or in the lower light cone. A uniqueness argument for the solutions of hyperbolic differential equations yields that both sides of (2.102) must coincide.

Next, we specify the unitary transformations $U_{L}$ and $U_{R}$ : We fix the points $x$ and $y$. For any point $z$ on the line segment $\overline{x y}$, we chose $U_{L / R}(z)$ as

$$
U_{L / R}(z)=\operatorname{Pexp}\left(-i \int_{x}^{z} A_{L / R}^{j}(z-x)_{j}\right)
$$

The differential equation (2.91) yields that

$$
\begin{aligned}
(y-x)^{j} U_{c}(z)\left(\partial_{j} U_{c}(z)^{-1}\right) & =\mathrm{Pe}^{-i \int_{x}^{z} A_{c}^{k}(z-x)_{k}}(y-x)^{j} \frac{\partial}{\partial z^{j}} \mathrm{Pe}^{-i \int_{z}^{x} A_{c}^{k}(x-z)_{k}} \\
& =\mathrm{Pe}^{-i \int_{x}^{z} A_{c}^{k}(z-x)_{k}} i(y-x)_{j} A_{c}^{j}(z) \mathrm{Pe}^{-i \int_{z}^{x} A_{c}^{k}(x-z)_{k}} \\
& =i(y-x)_{j} U_{c}(z) A_{c}^{j}(z) U_{c}(z)^{-1} .
\end{aligned}
$$

Substituting into (2.98) gives

$$
\hat{A}_{L / R}^{j}(z)(y-x)_{j}=0 \quad \text { for } z \in \overline{x y} .
$$

Thus our choice of $U_{L}$ and $U_{R}$ makes the potentials $\hat{A}_{L}(z)$ and $\hat{A}_{R}(z), z \in \overline{x y}$, orthogonal to $(y-x)$. Notice, however, that since $Y_{L / R}$ in $(2.99)$ is arbitrary and independent of $U_{L / R}$, (2.103) gives no constraints for the dynamic mass matrices $\hat{Y}_{L / R}$.

We point out that we did not specify $U_{L / R}(z)$ outside the line segment $z \in \overline{x y}$; the unitary transformation $U_{L / R}$ may be arbitrary there. This means that also $\hat{A}_{L / R}$ is undetermined outside the line segment $\overline{x y}$. Especially, all the non-tangential derivatives of $\hat{A}_{L / R}(z), z \in \overline{x y}$, are undetermined. However, equation (2.103) does give constraints for the tangential derivatives. For example, differentiating (2.104) in the direction $(y-x)$ yields

$$
(y-x)^{j}(y-x)_{k} \partial_{j} \hat{A}_{L / R}^{k}(z)=0 \quad \text { for } \quad z \in \overline{x y} .
$$

We now consider the perturbation expansion 2.101). The light-cone expansion of all Feynman diagrams according to Theorem 2.5 gives a sum of terms of the form

$$
\begin{gathered}
\chi_{c} C(y-x)^{K} \hat{W}^{(0)}(x) \int_{x}^{y}\left[l_{1}, r_{1} \mid n_{1}\right] d z_{1} \hat{W}^{(1)}\left(z_{1}\right) \int_{z_{1}}^{y}\left[l_{2}, r_{2} \mid n_{2}\right] d z_{2} \hat{W}^{(2)}\left(z_{2}\right) \\
\cdots \int_{z_{\alpha-1}}^{y}\left[l_{\alpha}, r_{\alpha} \mid n_{\alpha}\right] d z_{\alpha} \hat{W}^{(\alpha)}\left(z_{\alpha}\right) \gamma^{J} S^{(h)}(x, y)
\end{gathered}
$$

where the factors $\hat{W}^{(\beta)}$ are given by

$$
\hat{W}^{(\beta)}=\left(\partial^{K_{a_{\beta}}} \square^{p_{a_{\beta}}} \hat{V}_{J_{a_{\beta}}, c_{a_{\beta}}}^{\left(a_{\beta}\right)}\right) \cdots\left(\partial^{K_{b_{\beta}}} \square^{p_{b_{\beta}}} \hat{V}_{J_{b_{\beta}}, c_{b_{\beta}}}^{\left(b_{\beta}\right)}\right) .
$$

Because of condition (2.104), all the contributions which are not phase-free vanish. Furthermore, according to Theorem 2.5, the contributions (2.106), 2.107) contain no tangential derivatives. Clearly, the derivatives in these formulas may have a component in direction of $(y-x)$; but the contribution of the derivatives transversal to $(y-x)$ uniquely determines the form of each derivative term. Therefore all the phase-free contributions of the form (2.106), (2.107) are independent in the sense that we have no algebraic relations 
between them. We conclude that, as long as the potentials $\hat{A}_{L / R}$ and $\hat{Y}_{L / R}$ are only specified by (2.98), (2.99), and (2.103), the light-cone expansion (2.106), 2.107) consists precisely of all phase-free contributions.

Next, we exploit the local transformation law (2.102) of the Green's functions: We solve this equation for $\tilde{s}$,

$$
\tilde{s}(x, y)=V(x) \hat{s}(x, y) V(y)^{*} .
$$

The transformation $U_{L / R}$ does not enter on the left side of this equation. Thus the right side of (2.108) is also independent of $U_{L / R}$. Especially, we conclude that the light-cone expansion of $\hat{s}(x, y)$ must be independent of the derivatives of $U_{L / R}$ along the line segment $\overline{x y}$. At first sight, this might seem inconsistent because the individual contributions (2.106),(2.107) do depend on the derivatives of $U_{L / R}$ (this is obvious if one substitutes (2.98) and (2.99) into (2.107) and carries out the derivatives with the Leibniz rule). The right way to understand the independence of $\hat{s}(x, y)$ on the derivatives of $U_{L / R}$ is that all derivative terms of $U_{L / R}$ cancel each other to every order on the light cone if the (finite) sum over all contributions (2.106) to the light-cone expansion of $\hat{s}(x, y)$ is carried out. Since we will form the sum over all contributions to the light-cone expansion in the end, it suffices to consider only those contributions to the light-cone expansion which contain no derivatives of $U_{L / R}$. This means that we can substitute (2.98) and (2.99) into (2.107), forget about the derivative term $i U_{L / R}\left(\partial^{j} U_{L / R}^{-1}\right)$ in (2.98), and pull the unitary transformations $U_{L / R}, U_{L / R}^{-1}$ out of the derivatives. In other words, we can replace $\hat{W}^{(\beta)}$, (2.107), by

$$
\hat{W}^{(\beta)}=U_{c_{a_{\beta}}}\left(\partial^{K_{a_{\beta}}} \square^{p_{a_{\beta}}} V_{J_{a_{\beta}}, c_{a_{\beta}}}^{\left(a_{\beta}\right)}\right) U_{d_{a_{\beta}}}^{-1} \cdots U_{c_{b_{\beta}}}\left(\partial^{K_{b_{\beta}}} \square^{p_{b_{\beta}}} V_{J_{b_{\beta}}, c_{b_{\beta}}}^{\left(b_{\beta}\right)}\right) U_{d_{b_{\beta}}}^{-1}
$$

with chiral indices $c_{a}, d_{a}=L / R$. The light-cone expansion for $\hat{s}(x, y)$ consists precisely of the sum of all phase-free contributions of the form (2.106), (2.109).

The chiralities $c_{a}, d_{a}$ of the unitary transformations $U_{L / R}, U_{L / R}^{-1}$ in (2.109) are determined by the rules (i) and (ii) (in Theorem 2.3) and by (2.98) and (2.99). According to the rule (ii), the indices $c_{a}$ and $c_{a+1}$ coincide iff $V^{(a)}$ is a chiral potential. According to (2.98) and (2.99), on the other hand, the indices $c_{a}$ and $d_{a}$ coincide iff $V^{(a)}=A_{L / R}$. We conclude that the indices $d_{a}$ and $c_{a+1}$ always coincide. Thus all the intermediate factors $U_{d_{a}} U_{c_{a+1}}$ give the identity, and (2.109) simplifies to

$$
\hat{W}^{(\beta)}=U_{c_{\beta}} W^{(\beta)} U_{d_{\beta}}^{-1}
$$

Furthermore, the chiralities $c_{\beta}$ and $d_{\beta}$ coincide if and only if $W^{(\beta)}$ contains an even number of dynamic mass matrices.

Finally, we substitute the light-cone expansion (2.106), (2.110) for $\hat{s}(x, y)$ into (2.108). This gives for the light-cone expansion of $\tilde{s}(x, y)$ a sum of expressions of the form

$$
\begin{gathered}
\chi_{c} C(y-x)^{K} U_{c}^{-1}(x)\left(U_{c_{0}} W^{(0)} U_{d_{0}}^{-1}\right)(x) \int_{x}^{y}\left[l_{1}, r_{1} \mid n_{1}\right] d z_{1}\left(U_{c_{1}} W^{(1)} U_{d_{1}}^{-1}\right)\left(z_{1}\right) \\
\ldots \int_{z_{\alpha-1}}^{y}\left[l_{\alpha}, r_{\alpha} \mid n_{\alpha}\right] d z_{\alpha}\left(U_{c_{\alpha}} W^{(0)} U_{d_{\alpha}}^{-1}\right)\left(z_{\alpha}\right) U_{c_{\alpha+1}}(y) \gamma^{J} S^{(h)}(x, y)
\end{gathered}
$$

where the sum runs over all phase-free contributions of this type. Similar to the considerations before (2.110), one sees that adjacent unitary transformations always have the 
same chirality. Thus (2.111) can be simplified to

$$
\begin{aligned}
\chi_{c} C & (y-x)^{K} W^{(0)}(x) \int_{x}^{y}\left[l_{1}, r_{1} \mid n_{1}\right] d z_{1} U_{c_{1}}(x)^{-1} U_{c_{1}}\left(z_{1}\right) W^{(1)} \\
& \ldots \int_{z_{\alpha-1}}^{y}\left[l_{\alpha}, r_{\alpha} \mid n_{\alpha}\right] d z_{\alpha} U_{c_{\alpha}}\left(z_{\alpha-1}\right)^{-1} U_{c_{\alpha}}\left(z_{\alpha}\right) W^{(0)}\left(z_{\alpha}\right) U_{c_{\alpha+1}}\left(z_{\alpha}\right)^{-1} \\
& \times U_{c_{\alpha+1}}(y) \gamma^{J} S^{(h)}(x, y),
\end{aligned}
$$

whereby the indices $c_{a}$ satisfy the rule (II) of Def. 2.9. According to (2.103), the factors $U_{c}^{-1}(.) U_{c}($.$) coincide with the ordered exponentials in (2.96), which concludes the proof.$

For clarity, we point out that all the constructions following Def. 2.9 are based on the lightcone expansion of Theorem 2.5. It is essential for the statement of Theorem 2.10 that the phase-free contributions contain no tangential derivatives. If we had worked with the light-cone expansion of Theorem 2.3 (instead of Theorem 2.5), the light-cone expansion of $\hat{s}(x, y)$ would not have consisted of all the phase-free contributions to the light-cone expansion. For example, a line integral containing the tangential derivative (2.105) would vanish, although it is phase-free. As a consequence of this problem, the whole construction would break down.

The introduction of the phase-free and phase-inserted contributions has simplified the light-cone expansion of the Green's functions considerably: Assume that we want to perform the light-cone expansion to some given order on the light cone. Then we first calculate the phase-free contribution to the light-cone expansion; according to Proposition 2.8, this gives only a finite number of terms. Using the rules of Def. 2.9, we can easily construct the corresponding phase-inserted contributions. According to Theorem 2.10, this finite number of phase-inserted contributions gives precisely the light-cone expansion of the Green's functions to the desired order on the light cone. This procedure is called the reduction to the phase-free contribution.

\subsection{Calculation of the Phase-Free Contribution}

According to the reduction to the phase-free contribution, it remains to calculate the phase-free contribution to any given order on the light cone. Although this is still a very complicated problem, we know from Proposition 2.8 that we only get a finite number of terms. This makes it possible to use a computer algebra program for the calculation. The author has developed the $\mathrm{C}++$ program "class_commute" specifically for this problem. It generates explicit formulas for the phase-free contribution to any order on the light cone. We now outline how this program works, without entering implementation details?.

All the objects occurring in the calculation (like integrals, partial derivatives, Laplacians, potentials, Dirac matrices, etc.) are described by different data structures (classes in $\mathrm{C}++$ ). Formulas are built up as sums of lists of these data structures. The calculation is performed by manipulating the lists. More precisely, this works as follows: Each data structure carries an ordering number. At the beginning of the computation, the lists are disordered in the sense that their elements do not occur with increasing ordering numbers. For ordering the lists, the program iteratively commutes adjacent elements of a list. Each

\footnotetext{
${ }^{2}$ The commented source code of the program "class_commute" is available from the author on request. It is an extension of the program used in [2] for the light-cone expansion to first order in the external potential.
} 
commutation is performed by a function of the program which is specific to the particular pair of data structures; i.e., there is a function for commuting a partial derivative with a potential, a function for commuting two Dirac matrices, etc. (this is easily implemented in $\mathrm{C}++$ using virtual class functions). The data structures and commutation rules are designed in such a way that, after the ordering process has come to an end, the lists consist of the desired formulas of the light-cone expansion.

The main advantage of this implementation with commutation rules is that the programmer must only think of the calculation on a "local" scale by telling the computer the rules for commuting a given pair of data structures. Furthermore, this gives a convenient segmentation of the computer program into small, independent parts, which can be written and debugged separately. As soon as all commutation rules are specified correctly, the program can perform the whole calculation "globally" by recursively applying the commutation rules. Compared to standard computer algebra packages like e.g. Mathematica or Maple, this concept is very flexible and efficient.

For the more complex manipulations like partial integrations and the handling of tensor indices, the program uses a so-called "message pipe," through which the objects in the formulas can pass information to other objects (this is again implemented with virtual class functions). In this way, the elements of the lists can exchange data and give commands to each other. This allows a convenient coordination of the formula manipulations.

The calculation rules of the program "class_commute" are very similar to the construction steps described in the proofs of Theorem 2.3 and Theorem 2.5. The only difference is that, according to our implementation as commutation rules, the calculation does not follow the same strict and clear order as in Subsection 2.1. Basically, one may think of the construction steps of Theorem 2.3 and Theorem 2.5 as being performed simultaneously in a disordered way, whereby the program ensures that all rules are applied consistently.

Some formulas generated by the program "class_commute" are compiled in the appendix; they give a picture of the leading singularities of $\tilde{s}(x, y)$ on the light cone. We remark that "class_commute" was also a valuable tool for finding and checking the combinatorial results of Theorem 2.3, Lemma 2.4, and Theorem 2.5.

\section{The Light-Cone Expansion of the Dirac Sea}

In this section, we shall perform the light-cone expansion of the fermionic projector as defined in [1]. In Subsection 3.1, we establish a formal analogy between the light-cone expansions of the Dirac sea and of the Green's functions. This residual argument allows us to use the results of the previous section also for the fermionic projector. However, the analogy between the Dirac sea and the Green's functions cannot be extended beyond a purely formal level. The basic reason is that, in contrast to the Green's functions, the Dirac sea is a non-causal object. This is developed in detail in Subsections 3.2 and 3.3 .

We point out that, in this section, we do not work with the dynamic mass matrices $Y_{L / R}(x),(2.8)$. The reason is that, for the Dirac sea, the regularity conditions of Lemma 1.1 are necessary for the contributions to the perturbation expansion to be well-defined. Working with the dynamic mass matrices, however, implies that we consider the potential $B$, (2.10), as the perturbation of the Dirac operator; but $B$ does in general not go to zero at infinity. For our notation, the reader is referred to [1]. 


\subsection{The Residual Argument}

We begin by describing how the light-cone expansion of the Green's functions can be understood in momentum space. Apart from giving a different point of view, this allows us to get a connection to the light-cone expansion of the Dirac sea. For clarity, we begin with the special case $m Y=0$ of zero fermion mass. This case is particularly simple because then $B=\mathcal{B}$, so that the perturbation expansions (2.1) and (2.12) coincide. This is sufficient to explain the basic construction; the extension to $m Y \neq 0$ will later be accomplished by a general argument. Furthermore, we only consider the advanced Green's function; for the retarded Green's function, the calculation is analogous.

Suppose that we want to perform the light-cone expansion of the $k^{\text {th }}$ order contribution to the perturbation series $(2.1)=(2.12)$. We write the contribution as a multiple Fourier integral,

$$
\begin{aligned}
& \left(\left(-s^{\vee} B\right)^{k} s^{\vee}\right)(x, y) \\
& \quad=\int \frac{d^{4} p}{(2 \pi)^{4}} \int \frac{d^{4} q_{1}}{(2 \pi)^{4}} \cdots \int \frac{d^{4} q_{k}}{(2 \pi)^{4}} \Delta s^{\vee}\left(p ; q_{1}, \ldots, q_{k}\right) e^{-i\left(p+q_{1}+\cdots+q_{k}\right) x+i p y}
\end{aligned}
$$

where the distribution $\Delta s^{\vee}\left(p ; q_{1}, \ldots, q_{k}\right)$ is the Feynman diagram in momentum space,

$$
\begin{gathered}
\Delta s^{\vee}\left(p ; q_{1}, \ldots, q_{k}\right)=(-1)^{k} s^{\vee}\left(p+q_{1}+\cdots+q_{k}\right) \tilde{B}\left(q_{k}\right) s^{\vee}\left(p+q_{1}+\cdots+q_{k-1}\right) \tilde{B}\left(q_{k-1}\right) \\
\cdots \tilde{B}\left(q_{2}\right) s^{\vee}\left(p+q_{1}\right) \tilde{B}\left(q_{1}\right) s^{\vee}(p)
\end{gathered}
$$

( $\tilde{B}$ denotes the Fourier transform of the potential $B$, and $s^{\vee}(p)$ is the multiplication operator in momentum space). For the arguments of the Green's functions, we introduce the abbreviation

$$
p_{0}:=p \quad \text { and } \quad p_{l}:=p+q_{1}+\cdots+q_{l}, \quad 1 \leq l \leq k .
$$

Substituting the explicit formulas (2.3) and (2.15) into (3.2), we obtain

$$
\begin{aligned}
& \Delta s^{\vee}\left(p ; q_{1}, \ldots, q_{k}\right)=(-1)^{k} \not p_{k} \tilde{B}\left(q_{k}\right) \not p_{k-1} \cdots \not p_{1} \tilde{B}\left(q_{1}\right) \not p_{0} \\
& \quad \times \lim _{0<\varepsilon \rightarrow 0} \frac{1}{\left(p_{k}\right)^{2}-i \varepsilon p_{k}^{0}} \frac{1}{\left(p_{k-1}\right)^{2}-i \varepsilon p_{k-1}^{0}} \cdots \frac{1}{\left(p_{0}\right)^{2}-i \varepsilon p_{0}^{0}} .
\end{aligned}
$$

We now expand the Klein-Gordon Green's functions in (3.4) with respect to the momenta $p_{l}-p$. If we expand the terms $i \varepsilon p_{l}^{0}$ with a geometric series,

$$
\frac{1}{\left(p_{l}\right)^{2}-i \varepsilon p_{l}^{0}}=\sum_{n=0}^{\infty} \frac{\left(i \varepsilon\left(p_{l}^{0}-p^{0}\right)\right)^{n}}{\left(\left(p_{l}\right)^{2}-i \varepsilon p^{0}\right)^{1+n}}
$$

all contributions with $n \geq 1$ contain factors $\varepsilon$ and vanish in the limit $\varepsilon \rightarrow 0$. Therefore we must only expand with respect to the parameters $\left(\left(p_{l}\right)^{2}-p^{2}\right)$. This gives, again with geometric series,

$$
\begin{aligned}
& \Delta s^{\vee}\left(p ; q_{1}, \ldots, q_{k}\right)=(-1)^{k} \not p_{k} \tilde{B}\left(q_{k}\right) \not p_{k-1} \cdots \not p_{1} \tilde{B}\left(q_{1}\right) \not p_{0} \\
& \quad \times \sum_{n_{1}, \ldots, n_{k}=0}^{\infty}\left(p^{2}-p_{k}^{2}\right)^{n_{k}} \cdots\left(p^{2}-p_{1}^{2}\right)^{n_{1}} \lim _{0<\varepsilon \rightarrow 0} \frac{1}{\left(p^{2}-i \varepsilon p^{0}\right)^{1+k+n_{1}+\cdots+n_{k}}}
\end{aligned}
$$


Rewriting the negative power of $\left(p^{2}-i \varepsilon p^{0}\right)$ as a mass-derivative,

$$
\begin{aligned}
& \frac{1}{\left(p^{2}-i \varepsilon p^{0}\right)^{1+k+n_{1}+\cdots+n_{k}}} \\
& \quad=\frac{1}{\left(k+n_{1}+\cdots+n_{k}\right) !}\left(\frac{d}{d a}\right)^{k+n_{1}+\cdots+n_{k}} \frac{1}{p^{2}-a-i \varepsilon p^{0}}{ }_{\mid a=0},
\end{aligned}
$$

we obtain a formula containing only one Green's function. Namely, using the notation (2.13),

$$
\begin{aligned}
& \Delta s^{\vee}\left(p ; q_{1}, \ldots, q_{k}\right)=(-1)^{k} \not p_{k} \tilde{B}\left(q_{k}\right) \not p_{k-1} \cdots \not p_{1} \tilde{B}\left(q_{1}\right) \not p_{0} \\
& \quad \times \sum_{n_{1}, \ldots, n_{k}=0}^{\infty} \frac{1}{\left(k+n_{1}+\cdots+n_{k}\right) !}\left(p^{2}-p_{k}^{2}\right)^{n_{k}} \cdots\left(p^{2}-p_{1}^{2}\right)^{n_{1}} S^{\vee\left(k+n_{1}+\cdots+n_{k}\right)}(p) .
\end{aligned}
$$

This is the basic equation for the light-cone expansion of the Green's functions in momentum space. Similar to the light-cone expansion of the previous section, (3.6) involves the differentiated Green's functions $S^{\vee(.)}$. It remains to transform the polynomials in the momenta $p_{0}, \ldots, p_{k}$ until getting a connection to the nested line integrals of, say, Theorem 2.3: Substituting (3.3), we rewrite (3.6) in terms of the momenta $p, q_{1}, \ldots, q_{k}$ and multiply out. Furthermore, we simplify the Dirac matrices with the anti-commutation rules (2.53). This gives for (3.6) a sum of terms of the form

$$
\chi_{c} C \gamma^{I} q_{k}^{I_{k}} \cdots q_{1}^{I_{1}} \tilde{V}_{J_{k}, c_{k}}^{(k)}\left(q_{k}\right) \cdots \tilde{V}_{J_{1}, c_{1}}^{(1)}\left(q_{1}\right) p^{L} S^{\vee(h)}(p) \quad(h \geq[|L| / 2]),
$$

where the tensor indices of the multi-indices $I, I_{l}, J_{l}$, and $L$ are contracted with each other (similar to the notation of Theorem 2.3, the factors $\tilde{V}_{J_{l}, c_{l}}^{(l)}$ stand for the individual potentials of $\tilde{B})$. If tensor indices of the power $p^{L}$ are contracted with each other, we can iteratively eliminate the corresponding factors $p^{2}$ with the rule (2.14), more precisely

$$
p^{2} S^{\vee(h)}(p)=h S^{\vee(h-1)}(p) \quad(h \geq 1) .
$$

Thus we can arrange that the tensor indices of $p^{L}$ in (3.7) are all contracted with tensor indices of the factors $\gamma^{I}, q_{l}^{I_{l}}$, or $\tilde{V}_{J_{l}, c_{l}}^{(l)}$. By iteratively applying the differentiation rule (2.16), we can now rewrite the power $p^{L}$ in (3.7) with $p$-derivatives, e.g.

$$
\begin{aligned}
p_{j} p_{k} S^{\vee(2)}(p) & =-\frac{1}{2} p_{j} \frac{\partial}{\partial p^{k}} S^{\vee(1)}(p)=-\frac{1}{2} \frac{\partial}{\partial p^{k}}\left(p_{j} S^{\vee(1)}(p)\right)+\frac{1}{2} g_{j k} S^{\vee(1)}(p) \\
& =\frac{1}{4} \frac{\partial^{2}}{\partial p^{j} \partial p^{k}} S^{(0)}(p)+\frac{1}{2} g_{j k} S^{(1)}(p)
\end{aligned}
$$

In this way, we obtain for $\Delta s^{\vee}\left(p ; q_{1}, \ldots, q_{k}\right)$ a sum of terms of the form

$$
\chi_{c} C \gamma^{I} q_{k}^{I_{k}} \cdots q_{1}^{I_{1}} \tilde{V}_{J_{k}, c_{k}}^{(k)}\left(q_{k}\right) \cdots \tilde{V}_{J_{1}, c_{1}}^{(1)}\left(q_{1}\right) \partial_{p}^{K} S^{\vee(h)}(p),
$$

whereby no tensor indices of the derivatives $\partial_{p}^{K}$ are contracted with each other. We substitute these terms into (3.1) and transform them to position space. Integrating the derivatives $\partial_{p}^{K}$ by parts gives factors $(y-x)^{K}$. The factors $q_{l}^{I_{l}}$, on the other hand, can be written as partial derivatives $\partial^{I_{l}}$ acting on the potentials $V^{(l)}$. More precisely, the term (3.9) gives after substitution into (3.1) the contribution

$$
\chi_{c} C i^{\left|I_{1}\right|+\cdots+\left|I_{k}\right|}(-i)^{|K|} \gamma^{I}\left(\partial^{I_{k}} V_{J_{k}, c_{k}}^{(k)}(x)\right) \cdots\left(\partial^{I_{1}} V_{J_{1}, c_{1}}^{(1)}(x)\right)(y-x)^{K} S^{\vee(h)}(x, y) \quad,
$$


where the tensor indices of the factor $(y-x)^{K}$ are all contracted with tensor indices of the multi-indices $I, I_{l}$, or $J_{l}$. The Feynman diagram $\left((-s B)^{k} s\right)(x, y)$ coincides with the sum of all these contributions.

This expansion has much similarity with the light-cone expansion of Theorem 2.3 . Namely, if one expands the nested line integrals in (2.32) in a Taylor series around $x$, one gets precisely the expansion into terms of the form (3.10). Clearly, the light-cone expansion of Theorem 2.3 goes far beyond the expansion (3.10), because the dependence on the external potential is described by non-local line integrals. Nevertheless, the expansion in momentum space (3.6) and subsequent Fourier transformation give an easy way of understanding in principle how the formulas of the light-cone expansion come about. We remark that, after going through the details of the combinatorics and rearranging the contributions (3.10), one can recover the Taylor series of the line integrals in (2.32). This gives an alternative method for proving Theorem 2.3. However, it is obvious that this becomes complicated and does not yield the most elegant approach (the reader interested in the details of this method is referred to [2], where a very similar technique is used for the light-cone expansion to first order in the external potential).

Our next aim is to generalize the previous construction. Since we must, similar to (3.5), rewrite a product of Green's functions as the mass-derivative of a single Green's function, we can only expect the construction to work if all Green's functions in the product (3.2) are of the same type (e.g. the construction breaks down for a "mixed" operator product containing both advanced and retarded Green's functions). But we need not necessarily work with the advanced or retarded Green's functions. Instead, we can use Green's functions with a different position of the poles in the complex $p^{0}$-plane: We consider the Green's functions

$$
s^{ \pm}(p)=\not p S_{a \mid a=0}^{ \pm}(p) \quad \text { with } \quad S^{ \pm}(p)=\lim _{0<\varepsilon \rightarrow 0} \frac{1}{p^{2}-a \mp i \varepsilon}
$$

and again use the notation (2.13),

$$
S^{ \pm(l)}=\left(\frac{d}{d a}\right)^{l} S_{a \mid a=0}^{ \pm}
$$

The perturbation expansion for these Dirac Green's functions is, similar to (2.1) or (2.12), given by the formal series

$$
\tilde{s}^{+}:=\sum_{n=0}^{\infty}\left(-s^{+} B\right)^{n} s^{+}, \quad \tilde{s}^{-}:=\sum_{n=0}^{\infty}\left(-s^{-} B\right)^{n} s^{-} .
$$

The light-cone expansion in momentum space is performed exactly as for the advanced and retarded Green's functions; we obtain in analogy to (3.1) and (3.6) the formula

$$
\begin{aligned}
& \left(\left(-s^{ \pm} B\right)^{k} s^{ \pm}\right)(x, y) \\
& \quad=\int \frac{d^{4} p}{(2 \pi)^{4}} \int \frac{d^{4} q_{1}}{(2 \pi)^{4}} \cdots \int \frac{d^{4} q_{k}}{(2 \pi)^{4}} \Delta s^{ \pm}\left(p ; q_{1}, \ldots, q_{k}\right) e^{-i\left(p+q_{1}+\cdots+q_{k}\right) x+i p y}
\end{aligned}
$$

with

$$
\begin{aligned}
& \Delta s^{ \pm}\left(p ; q_{1}, \ldots, q_{k}\right)=(-1)^{k} \not p_{k} \tilde{B}\left(q_{k}\right) \not p_{k-1} \cdots \not p_{1} \tilde{B}\left(q_{1}\right) \not p_{0} \\
& \quad \times \sum_{n_{1}, \ldots, n_{k}=0}^{\infty} \frac{1}{\left(k+n_{1}+\cdots+n_{k}\right) !}\left(p^{2}-p_{k}^{2}\right)^{n_{k}} \cdots\left(p^{2}-p_{1}^{2}\right)^{n_{1}} S^{ \pm\left(k+n_{1}+\cdots+n_{k}\right)} .
\end{aligned}
$$


Since $S^{ \pm}$are Green's functions of the Klein-Gordon equation, they clearly also satisfy the identity (3.8). Furthermore, the differentiation rule (2.16) is also valid for $S^{ \pm}$; namely

$$
\begin{aligned}
\frac{\partial}{\partial p^{j}} S^{ \pm(l)}(p) & =\left(\frac{d}{d a}\right)^{l} \lim _{0<\varepsilon \rightarrow 0} \frac{\partial}{\partial p^{j}}\left(\frac{1}{p^{2}-a \mp i \varepsilon}\right)_{\mid a=0} \\
& =\left(\frac{d}{d a}\right)^{l} \lim _{0<\varepsilon \rightarrow 0} \frac{-2 p_{j}}{\left(p^{2}-a \mp i \varepsilon\right)^{2}}{ }_{\mid a=0}=-2 p_{j} S^{ \pm(l+1)}(p) .
\end{aligned}
$$

Therefore we can, exactly as in (3.9), rewrite the power $p^{L}$ with $p$-derivatives. Thus the expansion (3.10) is valid in the same way for the Green's functions $s^{ \pm}$if one only replaces the index "V" in (3.10) by " \pm ". As explained before, the expansion (3.10) is obtained from the light-cone expansion of Theorem 2.3 by expanding the potentials around the spacetime point $x$. Since the formulas of the light-cone expansion are uniquely determined by this Taylor expansion, we immediately conclude that the statement of Theorem 2.3 is also valid for the $k^{\text {th }}$ order contribution to the perturbation expansion (3.12) if the factor $S^{(h)}$ in (2.32) stands more generally for $S^{+(h)}$ or $S^{-(h)}$, respectively. This simple analogy between the formulas of the light-cone expansion of the Feynman diagrams $\left(\left(-s^{\vee / \wedge} B\right)^{k} s^{\vee / \wedge}\right)$ and $\left(\left(-s^{ \pm} B\right)^{k} s^{ \pm}\right)$, which is obtained by changing the position of the poles of the free Green's functions in momentum space, is called the residual argument.

After these preparations, we come to the fermionic projector in the general case $m Y \neq$ 0 . We want to extend the light-cone expansion to an object $\tilde{P}^{\text {res }}$ being a perturbation of the free fermionic projector. Our method is to define $\tilde{P}^{\text {res }}$ in such a way that it can be easily expressed in terms of the Green's functions $\tilde{s}^{\vee}, \tilde{s}^{\wedge}, \tilde{s}^{+}$, and $\tilde{s}^{-}$. The light-cone expansion of the Green's functions then immediately carries over to $\tilde{P}^{\text {res }}$. We denote the lower mass shell by $T_{a}$, i.e. in momentum space

$$
T_{a}(q)=\Theta\left(-q^{0}\right) \delta\left(q^{2}-a\right)
$$

and set

$$
T^{(l)}=\left(\frac{d}{d a}\right)^{l} T_{a \mid a=0}
$$

Furthermore, we introduce, exactly as in [1], the series of operator products

$$
b^{<}=\sum_{k=0}^{\infty}(-s \mathcal{B})^{k}, \quad b=\sum_{k=0}^{\infty}(-\mathcal{B} s)^{k} \mathcal{B}, \quad b^{>}=\sum_{k=0}^{\infty}(-\mathcal{B} s)^{k}
$$

Def. 3.1 The residual fermionic projector $\tilde{P}^{r e s}(x, y)$ is defined by

$$
\tilde{P}^{r e s}(x, y)=\frac{1}{2} X\left(\tilde{p}^{r e s}-\tilde{k}\right)(x, y)
$$

where the operators $\tilde{p}^{\text {res }}$ and $\tilde{k}$ are given by the perturbation series

$$
\begin{aligned}
\tilde{p}^{r e s} & =\sum_{\beta=0}^{\infty}(-i \pi)^{2 \beta} b^{<} p(b p)^{2 \beta} b^{>} \\
\tilde{k} & =\sum_{\beta=0}^{\infty}(-i \pi)^{2 \beta} b^{<} k(b k)^{2 \beta} b^{>} .
\end{aligned}
$$


Proposition 3.2 (formal light-cone expansion of the residual fermionic projector) The results of Section 2 also apply to the residual fermionic projector. More precisely, the light-cone expansion of Theorem 2.5 holds for $\tilde{P}^{\text {res }}(x, y)$ if we replace $S^{(h)}$ by $T^{(h)}$ and multiply the formulas of the light-cone expansion from the left with the chiral asymmetry matrix X. According to Theorem 2.5, all tangential derivatives can be integrated by parts. With Def. 2.7, Def. 2.9, and Theorem 2.10, the light-cone expansion can be reduced to the phase-free contribution. According to Proposition 2.8, the phase-free contribution consists, to every order $\sim T^{(h)}$, of only a finite number of terms.

Proof: First of all, we must generalize the residual argument to the case $m Y \neq 0$ of massive fermions. According to (2.1) and (2.12), there are two equivalent perturbation series for $\tilde{s}^{\vee}$,

$$
\begin{aligned}
\tilde{s}^{\vee} & =\sum_{k=0}^{\infty}\left(-s^{\vee} \mathcal{B}\right)^{k} s^{\vee} \\
& =\sum_{k=0}^{\infty}\left(-s_{m=0}^{\vee} B\right)^{k} s_{m=0}^{\vee} .
\end{aligned}
$$

In both perturbation series, each summand is a well-defined tempered distribution (this follows from the smoothness of $B, \mathcal{B}$ and from the causality of the perturbation expansion). In Section 2, we developed the light-cone expansion from the series in (3.19). But by arranging the contributions to this light-cone expansion in powers of the potential $\mathcal{B}$, one also obtains formulas for the light-cone expansion of every Feynman diagram of the perturbation series (3.18). For the Green's functions $s^{ \pm}$, we have similar perturbation expansions

$$
\begin{aligned}
\tilde{s}^{ \pm} & =\sum_{k=0}^{\infty}\left(-s^{ \pm} \mathcal{B}\right)^{k} s^{ \pm} \\
& =\sum_{k=0}^{\infty}\left(-s_{m=0}^{ \pm} B\right)^{k} s_{m=0}^{ \pm} .
\end{aligned}
$$

Since the support of the distributions $s^{ \pm}(x, y)$ does not vanish outside the light cone, we now need the conditions of Lemma 1.1 on the decay of the potentials at infinity. According to our assumptions on $\mathcal{B}$, each summand of the perturbation expansion (3.20) is a welldefined distribution. The potential $B$, however, does in general not decay at infinity; thus the Feynman diagrams of the perturbation expansion (3.21) are ill-defined. This is a problem, especially because in our above consideration, the residual argument was derived for the Feynman diagrams of the expansions (3.19) and (3.21). The solution to this problem is an approximation argument using the "causality" of the formulas of the light-cone expansion: We consider a smooth function $\eta_{R}(x)$ which is equal to one inside the ball of radius $R$ around the origin and vanishes outside the ball of radius $2 R$ (in $\mathbb{R}^{4}$ equipped with the standard Euclidean metric). Then the potential $\eta_{R} B$ has compact support and, according to Lemma 1.1, the Feynman diagrams

$$
\left(\left(-s_{m=0}^{ \pm} \eta_{R} B\right)^{k} s_{m=0}^{ \pm}\right)(x, y)
$$

are well-defined. We can apply the above residual argument for $m Y=0$; this yields formulas of the light-cone expansion in terms of the potential $\left(\eta_{R} B\right)$ and its partial derivatives. 
Since the potential enters into the formulas of the light-cone expansion only along the convex line $\overline{x y}$, we can, by taking the limit $R \rightarrow \infty$, remove the cutoff function $\eta_{R}$ from these formulas. This limiting process shows that the summands of the perturbation series in (3.21) make mathematical sense in terms of the light-cone expansion. By reordering the contributions, we immediately also get formulas for the light-cone expansion of the Feynman diagrams of the perturbation series (3.20). The analogy between the light-cone expansions of the Feynman diagrams of the perturbation series (3.18) and (3.20) finally yields the extension of the residual argument to a general mass matrix $m Y$.

Evaluating the poles in (2.15) and (3.12) in the complex $p^{0}$-plane gives (using the formula $\left.\lim _{0<\varepsilon \rightarrow 0}\left((x-i \varepsilon)^{-1}-(x+i \varepsilon)^{-1}\right)=2 \pi i \delta(x)\right)$ the relations

$$
\begin{array}{ll}
s^{\vee}=s+i \pi k, & s^{\wedge}=s-i \pi k \\
s^{+}=s+i \pi p, & s^{-}=s-i \pi p,
\end{array}
$$

where $s$ denotes as in [1] the arithmetic mean of the advanced and retarded Green's functions,

$$
s=\frac{1}{2}\left(s^{\vee}+s^{\wedge}\right)
$$

We substitute (3.23) and (3.24) into the perturbation series (2.1), (3.20) and multiply out. After rearranging the sums, one sees that the series (3.16) and (3.17) can be written as

$$
\tilde{p}^{\mathrm{res}}=\frac{1}{2 \pi i}\left(\tilde{s}^{+}-\tilde{s}^{-}\right) \quad \text { and } \quad \tilde{k}=\frac{1}{2 \pi i}\left(\tilde{s}^{\vee}-\tilde{s}^{\wedge}\right),
$$

respectively (see [1], proof of Theorem 3.2] for the details of the combinatorics). According to the residual argument, all Green's functions have a light-cone expansion according to Theorem 2.3. By substituting into (3.25), this light-cone expansion immediately generalizes to $\tilde{p}^{\text {res }}$ and $\tilde{k}$. Using (3.15), we conclude that Theorem 2.3 is also valid for $\tilde{P}^{\text {res }}$ after

the replacement $S^{(h)} \rightarrow T^{(h)}$ and multiplication with the chiral asymmetry matrix. Since the results of Theorem 2.5, Proposition 2.8, and Theorem 2.10 are obtained merely by manipulating and rearranging the formulas of the light-cone expansion, they also hold for the residual fermionic projector.

We point out that the argumentation in this subsection was only formal in the sense that we did not care about the convergence of the infinite sums. Also, the approximation argument in the proof of Proposition 3.2 requires a mathematical justification. Furthermore, the derivative (3.14) is ill-defined because, for $a=0$, the mass shell degenerates to the cone $\left\{q^{2}=0, q^{0}<0\right\}$, which is not differentiable at $q=0$. We postpone the mathematical analysis of these problems to Subsection 3.3.

\subsection{The Non-Causal High Energy Contribution}

Unfortunately, the residual fermionic projector $\tilde{P}^{\text {res }}$ of the previous subsection does not coincide with the fermionic projector $\tilde{P}$ of $[\mathbb{1}$,

$$
\tilde{P}(x, y)=\frac{1}{2} X(\tilde{p}-\tilde{k})(x, y) .
$$

The difference is that, instead of the operator $\tilde{p}^{\text {res }}$ in the residual fermionic projector (3.15), the fermionic projector (3.26) involves the operator $\tilde{p}$, which is formally given by

$$
\tilde{p} \stackrel{\text { formally }}{=} \sqrt{\tilde{k}^{2}} .
$$


Using an operator calculus method, this formal definition is made mathematically precise in [1] in terms of a perturbation series for $\tilde{p}$. Similar to (3.16), this perturbation expansion consists of a sum of operator products. But the operator products are more complicated; they also contain operators $k$ with some combinatorial factors (see [1] for details).

Before entering the mathematical analysis of the operator products, we point out that it is not just a matter of taste to take (3.26), and not (3.15), as the definition of the fermionic projector; only the definition $(\overline{3.26})$ makes physical sense. This comes as follows: As explained in 11], the operator $\tilde{k}$ generalizes the splitting of the solutions of the Dirac equation into solutions of positive and negative frequency to the case with interaction. The "generalized positive and negative frequency solutions" are given by the eigenstates of $\tilde{k}$ with positive and negative eigenvalue, respectively. The construction (3.27), (3.26) projects out all positive eigenstates of $\tilde{k}$; the operator $\frac{1}{2}(\tilde{p}-\tilde{k})$ consists precisely of all eigenstates of $\tilde{k}$ with negative eigenvalue. The residual fermionic projector (3.15), however, consists of a mixture of positive and negative eigenstates of $\tilde{k}$, which is not a reasonable physical concept.

We begin by giving the difference between the fermionic projector and the residual fermionic projector a name.

Def. 3.3 The non-causal high energy contribution $\tilde{P}^{h e}(x, y)$ to the fermionic projector is given by

$$
\tilde{P}^{h e}(x, y)=\tilde{P}(x, y)-\tilde{P}^{r e s}(x, y) .
$$

Clearly, this definition is only helpful if $\tilde{P}^{\text {he }}$ has some nice properties. The reason why the definition makes sense is that every contribution to the perturbation expansion of $\tilde{P}^{\text {he }}(x, y)$ is a smooth function. Thus the singular behavior of the fermionic projector on the light cone is completely described by the residual fermionic projector and its light-cone expansion, Proposition 3.2.

Theorem 3.4 The non-causal high energy contribution $\tilde{P}^{h e}(x, y)$ is, to every order in perturbation theory, a smooth function in $x$ and $y$.

Proof: The perturbation series [1, Theorem 4.1] defines $\tilde{p}$ as a sum of operator products of the form

$$
C_{n} \mathcal{B} C_{n-1} \mathcal{B} \cdots \mathcal{B} C_{0}
$$

where the factors $C_{l}$ coincide with either $k, p$, or $s$. The number of factors $k$ in these operator products is always even. If one replaces all factors $k$ by $p$, one gets precisely the perturbation series for $\tilde{p}^{\text {res }}$, 3.16) (this is verified using the details of the combinatorics in [1]). Therefore we can convert the perturbation series for $\tilde{p}$ into that for $\tilde{p}^{\text {res }}$ by iteratively replacing pairs of factors $k$ in the operator products by factors $p$. Thus the difference $\left(\tilde{p}-\tilde{p}^{\text {res }}\right)$ can, to every order in perturbation theory, be written as a finite sum of expressions of the form

$$
\begin{array}{rl}
C_{n} \mathcal{B} \cdots C_{b+1} & \mathcal{B}\left(p \mathcal{B} C_{b-1} \cdots C_{a+1} \mathcal{B} p\right. \\
& \left.-k \mathcal{B} C_{b-1} \cdots C_{a+1} \mathcal{B} k\right) \mathcal{B} C_{a-1} \cdots \mathcal{B} C_{0}
\end{array}
$$

where the factors $C_{l}$ again stand for $k, p$, or $s$. Since $\tilde{P}^{\text {he }}=\frac{1}{2} X\left(\tilde{p}-\tilde{p}^{\text {res }}\right)$, it suffices to show that (3.29) is a smooth function in position space.

We first simplify our problem: Once we have shown that the bracket in (3.29) is smooth and bounded in position space, the additional multiplications to the very left and right 
can be carried out by iteratively multiplying with $\mathcal{B}$ and forming the convolution with $C_{l}$, which again gives a smooth and bounded function in each step (notice that, according to the assumptions of Lemma 1.1, $\mathcal{B}$ decays sufficiently fast at infinity). Thus we must only consider the bracket in (3.29). We rewrite this bracket with the projectors $\frac{1}{2}(p-k)$ and $\frac{1}{2}(p+k)$ on the lower and upper mass shells,

$$
\begin{aligned}
& p \mathcal{B} C_{n-1} \cdots C_{1} \mathcal{B} p-k \mathcal{B} C_{n-1} \cdots C_{1} \mathcal{B} k \\
& \quad=\frac{1}{2}(p+k) \mathcal{B} C_{n-1} \cdots C_{1} \mathcal{B}(p-k)+\frac{1}{2}(p-k) \mathcal{B} C_{n-1} \cdots C_{1} \mathcal{B}(p+k) .
\end{aligned}
$$

For symmetry reasons, it suffices to show that the first summand of this decomposition,

$$
\left((p+k) \mathcal{B} C_{n-1} \cdots C_{1} \mathcal{B}(p-k)\right)(x, y),
$$

is smooth and bounded.

We proceed in momentum space. We say that a function $f(q)$ has rapid decay for positive frequency if it is $C^{1}$, bounded together with its first derivatives (i.e. $\sup |f|, \sup \left|\partial_{l} f\right|<$ $\infty$ ), and satisfies for every $\alpha>0$ the bounds

$$
\sup _{\omega>0, \vec{k} \in \mathbb{R}^{3}}\left|\omega^{\alpha} f(\omega, \vec{k})\right|, \sup _{\omega>0, \vec{k} \in \mathbb{R}^{3}}\left|\omega^{\alpha} \partial_{l} f(\omega, \vec{k})\right|<\infty .
$$

After setting $C_{0}=p-k$ and $C_{n}=p+k$, the operator product (3.30) is of the form (1.8). We choose a function $g$ with rapid decay for positive frequency and decompose the operator product in the form (1.12), (1.13). It follows by induction that the functions $F_{j}$ all have rapid decay for positive frequency: The induction hypothesis is obvious by setting $F_{0}=g$. The induction step is to show that for a function $F_{j-1}$ with rapid decay for positive frequency, the convolution

$$
F_{j}(\omega, \vec{k})=\int \frac{d \omega^{\prime}}{2 \pi} \int \frac{d \vec{k}^{\prime}}{(2 \pi)^{3}} \tilde{B}\left(\omega-\omega^{\prime}, \vec{k}-\vec{k}^{\prime}\right) C_{j-1}\left(\omega^{\prime}, \vec{k}^{\prime}\right) F_{j-1}\left(\omega^{\prime}, \vec{k}^{\prime}\right)
$$

also has rapid decay for positive frequency. In Lemma 1.1, it was shown that $F_{j}$ is $C^{1}$ and bounded together with its first derivatives. As a consequence, we must only establish the bounds (3.31) for $\omega>1$. Because of monotonicity $\omega^{\alpha}<\omega^{\beta}$ for $\alpha<\beta$ (and $\omega>1$ ), it furthermore suffices to show that there are arbitrarily large numbers $\alpha$ satisfying the bounds (3.31); we only consider $\alpha=2 n$ with $n \in \mathbb{N}$. For $\omega>1$ and $\omega^{\prime} \in \mathbb{R}$, we have the inequality

$$
\omega^{2 n} \leq\left(2 \omega^{\prime}\right)^{2 n} \Theta\left(\omega^{\prime}\right)+\left(2\left(\omega-\omega^{\prime}\right)\right)^{2 n},
$$

as is immediately verified by checking the three regions $\omega^{\prime} \leq 0,0<\omega^{\prime} \leq \omega / 2$, and $\omega^{\prime}>\omega / 2$. We combine this inequality with (3.32) and obtain for $\omega>1$ the estimate

$$
\begin{aligned}
& \left|\omega^{2 n} F_{j}(\omega, \vec{k})\right| \\
& \leq\left|\int \frac{d \omega^{\prime}}{(2 \pi)} \int \frac{d \vec{k}^{\prime}}{(2 \pi)^{3}} \tilde{B}\left(\omega-\omega^{\prime}, \vec{k}-\vec{k}^{\prime}\right) C_{j-1}\left(\omega^{\prime}, \vec{k}^{\prime}\right)\left[\left(2 \omega^{\prime}\right)^{2 n} \Theta\left(\omega^{\prime}\right) F_{j-1}\left(\omega^{\prime}, \vec{k}\right)\right]\right| \\
& \quad+\left|\int \frac{d \omega^{\prime}}{2 \pi} \int \frac{d \vec{k}^{\prime}}{(2 \pi)^{3}}\left[\left(2\left(\omega-\omega^{\prime}\right)\right)^{2 n} \tilde{B}\left(\omega-\omega^{\prime}, \vec{k}-\vec{k}^{\prime}\right)\right] C_{j-1}\left(\omega^{\prime}, \vec{k}^{\prime}\right) F_{j-1}\left(\omega^{\prime}, \vec{k}\right)\right| .
\end{aligned}
$$

According to the induction hypothesis, the square bracket in (3.33) is bounded together with its first derivatives. Since $\tilde{B}$ has rapid decay at infinity, the square bracket in (3.34) 
also has rapid decay at infinity. Thus both integrals in (3.33) and (3.34) satisfy the hypothesis considered in Lemma 1.1 for (1.10), and are therefore bounded. For estimating $\left|\omega^{2 n} \partial_{l} F_{j}\right|$, we differentiate (3.32) and obtain similar to (3.33), 3.34 the inequality

$$
\begin{aligned}
& \left|\omega^{2 n} \partial_{l} F_{j}(\omega, \vec{k})\right| \\
& \leq\left|\int \frac{d \omega^{\prime}}{2 \pi} \int \frac{d \vec{k}^{\prime}}{(2 \pi)^{3}} \partial_{l} \tilde{B}\left(\omega-\omega^{\prime}, \vec{k}-\vec{k}^{\prime}\right) C_{j-1}\left(\omega^{\prime}, \vec{k}^{\prime}\right)\left[\left(2 \omega^{\prime}\right)^{2 n} \Theta\left(\omega^{\prime}\right) F_{j-1}\left(\omega^{\prime}, \vec{k}\right)\right]\right| \\
& \quad+\left|\int \frac{d \omega^{\prime}}{d \omega} \int \frac{d \vec{k}^{\prime}}{(2 \pi)^{3}}\left[\left(2\left(\omega-\omega^{\prime}\right)\right)^{2 n} \partial_{l} \tilde{B}\left(\omega-\omega^{\prime}, \vec{k}-\vec{k}^{\prime}\right)\right] C_{j-1}\left(\omega^{\prime}, \vec{k}^{\prime}\right) F_{j-1}\left(\omega^{\prime}, \vec{k}\right)\right| .
\end{aligned}
$$

This concludes the proof of the induction step.

We have just shown that for a function $g$ with rapid decay for positive frequency, the function

$$
F_{n}(q)=\int \frac{d^{4} q_{1}}{(2 \pi)^{4}}\left(\mathcal{B} C_{n-1} \mathcal{B} \cdots \mathcal{B} C_{1} \mathcal{B} C_{0}\right)\left(q, q_{1}\right) g\left(q_{1}\right)
$$

has rapid decay for positive frequency. We now consider what this means for our operator product (3.30) in position space. For a given four-vector $y=\left(y^{0}, \vec{y}\right)$, we choose

$$
g(\omega, \vec{k})=\eta(\omega) e^{-i\left(\omega y^{0}-\vec{k} \vec{y}\right)},
$$

where $\eta$ is a smooth function with $\eta(\omega)=1$ for $\omega \leq 0$ and $\eta(\omega)=0$ for $\omega>1$ (this choice of $g$ clearly has rapid decay for positive frequency). Since the support of the factor $C_{0}=(p-k)$ is the lower mass cone $\left\{q^{2} \geq 0, q^{0} \leq 0\right\}, g(\omega, \vec{k})$ enters into the integral (3.35) only for negative $\omega$. But for $\omega \leq 0$, the cutoff function $\eta$ is identically one. Thus the integral (3.35) is simply a Fourier integral; i.e., with a mixed notation in momentum and position space,

$$
F_{n}(q)=\left(\mathcal{B} C_{n-1} \mathcal{B} \cdots \mathcal{B} C_{1} \mathcal{B}(p-k)\right)(q, y) .
$$

Next, we multiply from the left with the operator $(p+k)$,

$$
\left((p+k) \mathcal{B} C_{n-1} \mathcal{B} \cdots \mathcal{B} C_{1} \mathcal{B}(p-k)\right)(q, y)=(p+k)(q) F_{n}(q)
$$

Since $F_{n}$ has rapid decay for positive frequency and $(p+k)$ has its support in the upper mass cone $\left\{q^{2} \geq 0, q^{0}>0\right\}$, their product decays fast at infinity. More precisely,

$$
\left|q^{I}(p+k)(q) F_{n}(q)\right| \leq \operatorname{const}(I)(p+k)(q)
$$

for any multi-index $I$. As a consequence, the Fourier transform of (3.36) is even finite after multiplying with an arbitrary number of factors $q$, i.e.

$$
\left|\int \frac{d^{4} q}{(2 \pi)^{4}} q^{I}(p+k)(q) F_{n}(q) e^{-i q x}\right| \leq \operatorname{const}(I)<\infty
$$

for all $x$ and $I$. This shows that our operator product in position space (3.30) is bounded and, for fixed $y$, a smooth function in $x$ (with derivative bounds which are uniform in $y$ ). Similarly, one obtains that (3.30) is, for fixed $x$, a smooth function in $y$. We conclude that the distribution (3.30) is a smooth and bounded function.

We point out that $\tilde{P}^{\text {he }}(x, y)$ is a non-causal object in the sense that it does not only depend 
on the potential $\mathcal{B}$ in the "diamond" $\left(L_{x}^{\vee} \cap L_{y}^{\wedge}\right) \cup\left(L_{x}^{\wedge} \cap L_{y}^{\vee}\right)$ (with $L_{x}^{\vee}$ and $L_{x}^{\wedge}$ according to (2.2)), but on the external potential in the entire space-time. This becomes clear from the fact that the support of the operators $p\left(z_{1}, z_{2}\right)$ in the perturbation expansion for $\tilde{P}^{\text {he }}$ is the whole space $\left(z_{1}, z_{2}\right) \in \mathbb{R}^{4} \times \mathbb{R}^{4}$. Especially, it is not possible to express $\tilde{P}^{\text {he }}(x, y)$ similar to the formulas of the light-cone expansion in terms of the potential and its partial derivatives along the convex line $\overline{x y}$.

The non-causal high energy contribution is an effect of higher order perturbation theory; it vanishes to first order in the external potential [2]. According to the decomposition into terms of the form (3.30), it comes about because states on the upper and lower mass shell are mixed through multiplication with the potential. Qualitatively speaking, this mixing only becomes an important effect if the energy (i.e. frequency) of the external potential is high enough to overcome the energy difference between the states on the upper and lower mass shell. This gives the justification for the name "high energy" contribution.

\subsection{The Non-Causal Low Energy Contribution}

In this subsection, we will put the residual argument and the formal light-cone expansion of Proposition 3.2 on a satisfying mathematical basis. In order to explain what we precisely need to do, we first recall how the light-cone expansion of the Green's functions makes mathematical sense: Theorem 2.3 gives a representation of every Feynman diagram of the perturbation series (2.12) as an infinite sum of contributions of the form (2.32). According to the bound (2.36), there are, for any given $h$, only a finite number of possibilities to choose $I_{a}$ and $p_{a}$; as a consequence, we get, for fixed $h$, only a finite number of contributions (2.32). Thus we can write the light-cone expansion in the symbolic form

$$
\left((-s B)^{k} s\right)(x, y)=\sum_{h=-1}^{\infty} \sum_{\text {finite }} \cdots S^{(h)}(x, y),
$$

where '...' stands for a configuration of the $\gamma$-matrices and nested line integrals in (2.32). According to the explicit formula (2.5), the higher $a$-derivatives of $S_{a}(x, y)$ contain more factors $(y-x)^{2}$ and are thus of higher order on the light cone. This makes it possible to understand the infinite sum in (3.37) in terms of Def. 1.2; we can give it a mathematical meaning via the approximation by the finite partial sums (1.15). In Subsection 2.2, it is shown that understanding the light-cone expansion via these partial sums even makes it possible to explicitly carry out the sum over all Feynman diagrams.

According to Proposition 3.2, all the results of Section 2 are, on a formal level, also valid for the residual Dirac sea. We begin by considering the light-cone expansion of the individual Feynman diagrams in more detail. Similar to (3.37), the $k^{\text {th }}$ order contribution $\Delta P^{\text {res }}$ to the residual Dirac sea has an expansion of the form

$$
\Delta P^{\mathrm{res}}(x, y)=\sum_{h=-1}^{\infty} \sum_{\text {finite }} \cdots T^{(h)}(x, y),
$$

where $T^{(h)}$ is the $a$-derivative (3.14) of the lower mass shell $T_{a},(3.13)$. In position space, $T_{a}$ has the explicit form

$$
\begin{aligned}
T_{a}(x, y)= & -\frac{1}{8 \pi^{3}} \lim _{0<\varepsilon \rightarrow 0} \frac{1}{\xi^{2}-i \varepsilon \xi^{0}} \\
& +\frac{a}{32 \pi^{3}} \lim _{0<\varepsilon \rightarrow 0}\left(\log \left(a \xi^{2}-i \varepsilon \xi^{0}\right)+i \pi+c\right) \sum_{j=0}^{\infty} \frac{(-1)^{j}}{j !(j+1) !} \frac{\left(a \xi^{2}\right)^{j}}{4^{j}}
\end{aligned}
$$




$$
-\frac{a}{32 \pi^{3}} \sum_{j=0}^{\infty} \frac{(-1)^{j}}{j !(j+1) !} \frac{\left(a \xi^{2}\right)^{j}}{4^{j}}(\Phi(j+1)+\Phi(j))
$$

with $\xi \equiv(y-x), c=2 C-\log 2$ with Euler's constant $C$, and the function

$$
\Phi(0)=0 \quad, \quad \Phi(n)=\sum_{k=1}^{n} \frac{1}{k} \quad \text { for } n \geq 1 .
$$

The logarithm in (3.39) is understood in the complex plane which is cut along the positive real axis (so that $\lim _{0<\varepsilon \rightarrow 0} \log (x+i \varepsilon)=\log |x|$ is real for $x>0$ ). Alternatively, one can avoid the complex logarithm using the formula

$$
\lim _{0<\varepsilon \rightarrow 0} \log \left(a \xi^{2}-i \varepsilon \xi^{0}\right)+i \pi=\log \left|a \xi^{2}\right|+i \pi \Theta\left(\xi^{2}\right) \epsilon\left(\xi^{0}\right)
$$

( $\epsilon$ is the step function $\epsilon(x)=1$ for $x \geq 0$ and $\epsilon(x)=-1$ otherwise); thus the complex logarithm describes both a logarithmic pole on the light cone and a constant contribution in the interior of the light cone. The basic difference between the light-cone expansions (3.37) and (3.38) is related to the logarithmic pole $\log |a|$ in (3.39). Namely, as a consequence of this logarithm, the higher $a$-derivatives of $T_{a}$ are not of higher order on the light cone. To the order $\mathcal{O}\left((y-x)^{2}\right)$, for example, one has

$$
\left(\frac{d}{d a}\right)^{n} T_{a}(x, y)=\frac{1}{32 \pi^{3}}\left(\frac{d}{d a}\right)^{n}(a \log |a|)+\mathcal{O}\left((y-x)^{2}\right) \quad(, n \geq 2) .
$$

This means that the infinite sum in (3.38) cannot be understood in terms of Def. 1.2; the number of summands is already infinite to a given order on the light cone. In our context of an expansion around $a=0$, the situation is even worse, because the $a$-derivatives of $T_{a}$ are singular for $a \rightarrow 0$ (as one sees e.g. in (3.40)). Thus not even the individual contributions to the light-cone expansion make mathematical sense. These difficulties arising from the logarithm in (3.39) are called the logarithmic mass problem (see [2] for a more detailed discussion in a slightly different setting). Since we know from Lemma 1.1 that the Feynman diagrams are all well-defined, the logarithmic mass problem is not a problem of the perturbation expansion, but shows that something is wrong with the light-cone expansion of Proposition 3.2.

In order to resolve the logarithmic mass problem, we first "regularize" the formal light-cone expansion by taking out the problematic $\log |a|$ term. By resumming the formal light-cone expansion, we then show that the difference between the residual Dirac sea and the "regularized" Dirac sea is a smooth function in position space. We introduce the notation

$$
\begin{aligned}
T_{a}^{\mathrm{reg}}(x, y) & =T_{a}(x, y)-\frac{a}{32 \pi^{3}} \log |a| \sum_{j=0}^{\infty} \frac{(-1)^{j}}{j !(j+1) !} \frac{\left(a \xi^{2}\right)^{j}}{4^{j}} \\
T^{\mathrm{reg}(l)} & =\left(\frac{d}{d a}\right)^{l} T_{a \mid a=0}^{\mathrm{reg}} \cdot
\end{aligned}
$$

Def. 3.5 The causal contribution $\tilde{P}^{\text {causal }}$ to the fermionic projector is obtained from the residual Dirac sea $\tilde{P}^{\text {res }}$ by replacing all factors $T^{(h)}$ in the formal light-cone expansion by $T^{r e g(h)}$. The non-causal low energy contribution $\tilde{P}^{l e}$ to the fermionic projector is given by

$$
\tilde{P}^{l e}(x, y)=\tilde{P}^{r e s}(x, y)-\tilde{P}^{c a u s a l}(x, y)
$$


By the replacement $T^{(h)} \rightarrow T^{\text {reg }(h)}$, the formal light-cone expansion of Proposition 3.2 becomes mathematically meaningful in terms of Def. 1.2. Thus we can restate this result as a theorem, leaving out the word "formal."

Theorem 3.6 (light-cone expansion of the causal contribution) The results of Section 8 also apply to the causal contribution to the fermionic projector. More precisely, the light-cone expansion of Theorem 2.3 holds for $\tilde{P}^{\text {causal }}$ if we replace $S^{(h)}$ by $T^{\text {reg }(h)}$, (3.49), and multiply the formulas of the light-cone expansion from the left with the chiral asymmetry matrix X. According to Theorem 2.5, all tangential derivatives can be integrated by parts. With Def. 2.7, Def. 2.9, and Theorem 2.19, the light-cone expansion can be reduced to the phase-free contribution. According to Proposition 2.8, the phase-free contribution consists, to every order $\mathcal{O}\left((y-x)^{2 g}\right)$ on the light cone, of only a finite number of terms.

We come to the analysis of the low energy contribution. In the following lemma, we reformulate the light-cone expansion of Theorem 2.3 in a way where the infinite sums are handled more explicitly.

Lemma 3.7 The light-cone expansion of the $k^{\text {th }}$ order contribution $\left((-s B)^{k} s\right)(x, y)$ to the perturbation series (2.19) can be written as a finite sum of expressions of the form

$$
\begin{gathered}
\sum_{n_{1}, \ldots, n_{k}=0}^{\infty} \frac{1}{n_{1} ! \cdots n_{k} !} \chi_{c} C(y-x)^{I} \int_{x}^{y}\left[a_{1}, b_{1}+n_{2}+\cdots+n_{k} \mid n_{1}\right] d z_{1} \partial_{z_{1}}^{I_{1}} \square_{z_{1}}^{n_{1}+q_{1}} V_{J_{1}, c_{1}}^{(1)}\left(z_{1}\right) \\
\times \int_{z_{1}}^{y}\left[a_{2}, b_{2}+n_{3}+\cdots+n_{k} \mid n_{2}\right] d z_{2} \partial_{z_{2}}^{I_{2}} \square_{z_{2}}^{n_{2}+q_{2}} V_{J_{2}, c_{2}}^{(2)}\left(z_{2}\right) \\
\quad \cdots \int_{z_{k-1}}^{y}\left[a_{k}, b_{k} \mid n_{k}\right] d z_{k} \partial_{z_{k}}^{I_{k}} \square_{z_{k}}^{n_{k}+q_{k}} V_{J_{k}, c_{k}}^{(k)}\left(z_{k}\right) \gamma^{J} S^{\left(r+n_{1}+\cdots+n_{k}\right)}(x, y) \quad \cdot \quad(3.43)
\end{gathered}
$$

In this formula, we use for the chiral and tensor indices the same notation as in Theorem 2.3; $a_{l}, b_{l}$, and $q_{l}$ are non-negative integers. The parameters $r$ and $b_{l}$ satisfy the bounds

$$
\begin{aligned}
r & \leq k-|I| \\
b_{l} & \geq r-l+|I|, \quad 1 \leq l \leq k .
\end{aligned}
$$

Proof: The form of the expression (3.43) is straightforward if one keeps track of the infinite sums in the inductive construction of Theorem 2.3; it is also obvious that we only get a finite number of such expressions. The only point which needs an explanation is how one can arrange that all infinite sums are of the form

$$
\sum_{n=0}^{\infty} \frac{1}{n !}[., . \mid n] \square^{n} \ldots .
$$

For this, we must manipulate the sums when then Laplacian $\square^{n}$ is carried out after (2.41). Whenever a Laplacian acts on a factor $(y-x)^{I}$, we shift the summation index by one. More precisely, in the case of one factor $(y-x)$, we use the transformation

$$
\begin{aligned}
& \sum_{n=0}^{\infty} \frac{1}{n !}[a, b \mid n] \square_{x}^{n}(y-x)^{i} f_{(n)}(x)=\sum_{n=0}^{\infty} \frac{1}{n !}[a, b \mid n]\left((y-x)^{i} \square_{x}^{n} f_{(n)}-2 n \partial^{i} \square^{n-1} f_{(n)}\right) \\
& \quad=(y-x)^{i} \sum_{n=0}^{\infty} \frac{1}{n !}[a, b \mid n] \square_{x}^{n} f_{(n)}(x)-2 \sum_{n=0}^{\infty} \frac{1}{n !}[a+1, b+1 \mid n] \square_{x}^{n} \partial^{i} f_{(n+1)}(x)
\end{aligned}
$$


(where $f_{(n)}$ denotes a function depending on $n$ ); in the general case of several factors $(y-x)$, we inductively apply (3.47).

It remains to show that the parameters $a_{l}, b_{l}$, and $q_{l}$ are non-negative, and that the inequalities (3.44) and (3.45) hold. For this, it suffices to consider the leading summand $n_{1}=\cdots=n_{k}=0$ of (3.43). Since this is a (special) contribution of the form (2.32), we can apply Theorem 2.3 with $a_{i}=l_{i}+n_{i}, b_{i}=r_{i}+n_{i}$, and $p_{i}=q_{i}$. It follows that $a_{l}, b_{l}$, and $q_{l}$ are non-negative. For the proof of the inequalities (3.44) and (3.45), we proceed inductively in the order $k$ of the perturbation theory. For $k=0$, we have $r=-1$ and $|I|=1$, so that the inequalities are satisfied. Assume that (3.44),(3.45) hold for a given $k$. We go through the construction steps of Theorem 2.3 using the index shift (3.47) and verify that (3.44) and (3.45) are also valid to $(k+1)^{\mathrm{st}}$ order:

For the proof of (3.44), we note that additional factors $(y-x)$ are generated at most once in the construction; namely, if the derivative $\not_{x}$ acts on $S^{(\hat{h})}$ in step 5). The parameter $r$ is only increased if either a Laplacian acts on the factor $(y-x)^{I}$ in step 3) (leading to the index shift $(3.47)$ ) or if the derivative $\not_{x}$ does not act on $S^{(\hat{h})}$ in step 5). In both cases, one loses at least one factor $(y-x)$. This gives (3.44).

For the proof of (3.45), we take the contribution (3.43) with $n_{1}=\cdots=n_{k}=0$ and apply the construction of Theorem 2.3. When computing the Laplacian $\square_{z}^{n}$ in step 3), we shift the index according to (3.47) whenever a Laplacian acts on a factor $(y-x)$. Denoting the number of index shifts by $s$, we get a finite number of terms of the form

$$
\begin{aligned}
\sum_{n=0}^{\infty} & \frac{1}{n !} \chi_{c} C i \not \partial_{x} S^{(\hat{r}+n)}(x, y) \int_{x}^{y}[s, r+s \mid 0] d z(y-x)^{\hat{I}} \partial_{z}^{I_{0}} \square^{n} V_{J_{0}}^{(0)}(z) \\
& \times \int_{z}^{y}\left[a_{1}, b_{1} \mid 0\right] d z_{1} \partial_{z_{1}}^{I_{1}} \square_{z_{1}}^{q_{1}} V_{J_{1}, c_{1}}^{(1)}\left(z_{1}\right) \cdots \int_{z_{k-1}}^{y}\left[a_{k}, b_{k} \mid 0\right] d z_{k} \partial_{z_{k}}^{I_{k}} \square_{z_{k}}^{q_{k}} V_{J_{k}, c_{k}}^{(k)}\left(z_{k}\right) \gamma^{J} .
\end{aligned}
$$

Each index shift decreases the number of factors $(y-z)$ and increments the order of the mass-derivative of the Green's function, thus

$$
|\hat{I}| \leq|I|-s, \quad \hat{r}=r+1+s \quad .
$$

It again suffices to consider the leading summand $n=0$; this is a contribution of the form (2.32). After extracting the factors $(y-x)$ in step 4), the parameter $b_{0}=r_{0}+n_{0}$ satisfies

$$
b_{0}=r+|\hat{I}|+s \stackrel{3.48)}{\geq} \hat{r}+|\hat{I}|-1 .
$$

The parameters $b_{l}, 1 \leq l \leq k$, remain unchanged in the construction; they are still the same as in (3.45),

$$
b_{l} \geq r-l+|I| \geq \hat{r}-(l+1)+|\hat{I}|, \quad l=1, \ldots, k .
$$

When the derivative $\not_{x}$ is carried out in step 5), either $r$ is decremented and $|\hat{I}|$ increased by one, or $|\hat{I}|$ is decreased. In steps 6 ) and 7), the transformations (2.56) and (2.57) may only decrease the sum $\hat{r}+|\hat{I}|$. We conclude that, after performing all the construction steps,

$$
b_{l} \geq \hat{r}-(l+1)+|\hat{I}|, \quad l=0, \ldots, k .
$$

The index shift $l \rightarrow l+1$ finally gives the inequalities (3.45) for $l=1, \ldots, k+1$. 
Theorem 3.8 The non-causal low energy contribution $\tilde{P}^{l e}(x, y)$ is, to every order in perturbation theory, a smooth function in $x$ and $y$.

Proof: We first outline our strategy: According to Def. 3.1, Proposition 3.2, and Def. 3.5, the $k^{\text {th }}$ order contribution to $\tilde{P}^{\text {le }}(x, y)$ is obtained from (3.37) by the replacement $S^{(h)} \rightarrow$ $\left(T^{(h)}-T^{\mathrm{reg}(h)}\right)$,

$$
\Delta P^{\mathrm{le}}(x, y)=X \sum_{h=-1}^{\infty} \sum_{\text {finite }} \cdots\left(T^{(h)}-T^{\mathrm{reg}(h)}\right)(x, y) .
$$

Because of the logarithmic mass problem, the infinite sum in (3.49) is ill-defined. In order to give (3.49) a mathematical meaning, we manipulate the infinite sum until recovering it as a formal Taylor series, which can be carried out explicitly. Finally, we show that the mathematical object $\Delta P^{\mathrm{le}}(x, y)$ obtained in this way is a smooth function in $x$ and $y$.

Consider the light-cone expansion of the Green's functions of Lemma 3.7. Since there are only a finite number of contributions of the form (3.43), we can restrict ourselves to one of them. In order to get the corresponding contribution to $\tilde{P}^{\text {le }}$, we replace the factor $S^{(h)}$ in (3.43) according to (3.49) by the operator $L^{(h)}:=T^{(h)}-T^{\text {reg }(h)}$, i.e. more explicitly

$$
L^{(h)}(x, y)=\left(\frac{d}{d a}\right)^{h} L_{a \mid a=0}
$$

with

$$
L_{a}(x, y)=\frac{a}{32 \pi^{3}} \log |a| \sum_{j=0}^{\infty} \frac{(-1)^{j}}{j !(j+1) !} \frac{\left(a(y-x)^{2}\right)^{j}}{4^{j}} .
$$

We can leave out the factor $(y-x)^{I}$ in $(3.43)$ and disregard the chiral asymmetry matrix $X$ in (3.49), because they are irrelevant as smooth functions. Furthermore, we can carry out the partial derivatives $\partial_{z_{l}}^{I_{l}}$ in (3.43) with the Leibniz rule. According to the chain rule (2.49), this may increase the parameters $b_{l}$; nevertheless, the inequalities (3.44) and (3.45) remain valid. We conclude that it suffices to consider the formal series

$$
\begin{aligned}
\Delta P^{\mathrm{le}}(x, y)= & \sum_{n_{1}, \ldots, n_{k}=0}^{\infty} \frac{1}{n_{1} ! \cdots n_{k} !} \int_{x}^{y}\left[a_{1}, b_{1}+n_{2}+\cdots+n_{k} \mid n_{1}\right] d z_{1} \square_{z_{1}}^{n_{1}} W_{1}\left(z_{1}\right) \\
& \cdots \int_{z_{k-1}}^{y}\left[a_{k}, b_{k} \mid n_{k}\right] d z_{k} \square_{z_{k}}^{n_{k}} W_{k}\left(z_{k}\right) L^{\left(r+n_{1}+\cdots+n_{k}\right)}(x, y)
\end{aligned}
$$

together with the bounds (3.44) and (3.45), where $W_{l}$ stand for partial derivatives of the potential $V_{J_{l}, c_{l}}^{(l)}$. Our task is to give this series a mathematical meaning and to show that it is a smooth function in $x$ and $y$.

We first consider the case that the potentials $W_{l}$ are plane waves,

$$
W_{l}(x)=e^{-i q_{l} x} .
$$

This allows us to explicitly carry out the Laplacians in (3.51), and we obtain

$$
\begin{aligned}
& \Delta P^{\mathrm{le}}(x, y)=\int_{x}^{y}\left[a_{1}, b_{1} \mid 0\right] d z_{1} e^{-i q_{1} z_{1}} \cdots \int_{z_{k-1}}^{y}\left[a_{k}, b_{k} \mid 0\right] d z_{k} e^{-i q_{k} z_{k}} \\
& \times \sum_{n_{1}, \ldots, n_{k}=0}^{\infty} \frac{1}{n_{1} ! \cdots n_{k} !}\left(\left(\alpha_{1}^{2}-\alpha_{1}\right) p_{1}^{2}\right)^{n_{1}}\left(\left(1-\alpha_{1}\right)\left(\alpha_{2}^{2}-\alpha_{2}\right) p_{2}^{2}\right)^{n_{2}} \\
& \quad \cdots\left(\left(1-\alpha_{1}\right)\left(1-\alpha_{2}\right) \cdots\left(1-\alpha_{k-1}\right)\left(\alpha_{k}^{2}-\alpha_{k}\right) p_{k}^{2}\right)^{n_{k}} L^{\left(r+n_{1}+\cdots+n_{k}\right)}(x, y)
\end{aligned}
$$


where $\alpha_{l}$ denote the integration variables of the line integrals (all running from zero to one), and where $p_{l}$ are the momenta

$$
p_{l}=q_{l}+\left(1-\alpha_{l}\right) q_{l+1}+\cdots+\left(1-\alpha_{l}\right)\left(1-\alpha_{l+1}\right) \cdots\left(1-\alpha_{k-1}\right) q_{k} .
$$

For fixed values of the parameters $\alpha_{1}, \ldots, \alpha_{k},(3.52)$ is a product of $k$ formal Taylor series. For example by using the continuation of the logarithm into the complex plane

$$
\log |a|=\frac{1}{2} \lim _{0<\varepsilon \rightarrow 0}(\log (a+i \varepsilon)+\log (a-i \varepsilon)+2 i \pi)
$$

(which is, as in (3.39), cut along the positive real axis), we can carry out these formal Taylor series and obtain

$$
\Delta P^{\mathrm{le}}(x, y)=\int_{x}^{y}\left[a_{1}, b_{1} \mid 0\right] d z_{1} e^{-i q_{1} z_{1}} \ldots \int_{z_{k-1}}^{y}\left[a_{k}, b_{k} \mid 0\right] d z_{k} e^{-i q_{k} z_{k}}\left(\frac{d}{d a}\right)^{r} L_{a}(x, y)
$$

with

$$
\begin{aligned}
a=\left(\alpha_{1}^{2}-\right. & \left.\alpha_{1}\right) p_{1}^{2}+\left(1-\alpha_{1}\right)\left(\alpha_{2}^{2}-\alpha_{2}\right) p_{2}^{2} \\
& +\cdots+\left(1-\alpha_{1}\right)\left(1-\alpha_{2}\right) \cdots\left(1-\alpha_{k-1}\right)\left(\alpha_{k}^{2}-\alpha_{k}\right) p_{k}^{2}
\end{aligned}
$$

and the momenta $p_{l}$ according to (3.53). This construction is helpful in two ways: all infinite sums have disappeared, and the mass parameter $a$ is now in general non-zero, so that the $a$-derivatives of $L_{a}$ are no longer singular.

After this preparation, we consider the case that the potentials $V^{(l)}$ in (3.43) are smooth and satisfy the conditions of Lemma 1.1 on the decay at infinity. Then the Fourier transform $\tilde{V}^{(l)}$ is $C^{2}$ and has rapid decay at infinity. Since the potentials $W^{(l)}$ are partial derivatives of $V^{(l)}$, their Fourier transforms $\tilde{W}_{l}$ are also $C^{2}$ and have rapid decay at infinity. The low energy contribution is obtained from (3.54) by integrating over the momenta $q_{l}$. More precisely,

$$
\begin{aligned}
& \Delta P^{\mathrm{le}}(x, y)=\left(\frac{d}{d b}\right)^{r} \int \frac{d^{4} q_{1}}{(2 \pi)^{4}} \cdots \int \frac{d^{4} q_{k}}{(2 \pi)^{4}} \tilde{W}_{1}\left(q_{1}\right) \cdots \tilde{W}_{k}\left(q_{k}\right) \\
& \quad \times \int_{x}^{y}\left[a_{1}, b_{1} \mid 0\right] d z_{1} e^{-i q_{1} z_{1}} \cdots \int_{z_{k-1}}^{y}\left[a_{k}, b_{k} \mid 0\right] d z_{k} e^{-i q_{k} z_{k}} L_{a+b}(x, y)_{\mid b=0},
\end{aligned}
$$

where the parameter $a$ depends on the momenta $q_{l}$ via (3.55) and (3.53). We must show that (3.56) is well-defined and depends smoothly on $x$ and $y$. Qualitatively speaking, we can view the $q_{l}$-integrations as a multiple convolution in the parameter $a$. These convolutions mollify $L_{a+b}$ in such a way that the $b$-derivatives can be carried out giving a smooth function in $x$ and $y$. Unfortunately, this "mollifying argument" is quite delicate. Complications arise from the fact that the $q_{l}$-integrals are multi-dimensional and that we must handle the additional line integrals over $\alpha_{l}$. The main problem is that the dependence of $a$ on the momenta $q_{l}$ becomes singular when the parameters $\alpha_{l}$ approach zero or one (see (3.55)). Because of these difficulties, we give the mollifying argument in detail. Equivalently to analyzing the regularity in the parameter $a$, one can take its Fourier transform,

$$
L_{a}(x, y)=\int_{-\infty}^{\infty} \frac{d \tau}{2 \pi} \tilde{L}_{\tau}(x, y) e^{-i \tau a}
$$

and study the decay properties in $\tau$ for $\tau \rightarrow \pm \infty$. We prefer working with the parameter $\tau$, because this is a bit easier and makes our argument clearer. 
In the first step of the mollifying argument, we transform the $q_{l}$-integrals into integrals over the momenta $p_{l}$. Since the transformation (3.53) is volume preserving, we get

$$
\begin{aligned}
& \Delta P^{\mathrm{le}}(x, y)=\left(\frac{d}{d b}\right)^{r} \int \frac{d^{4} p_{1}}{(2 \pi)^{4}} \cdots \int \frac{d^{4} p_{k}}{(2 \pi)^{4}} \\
& \quad \times \int_{x}^{y}\left[a_{1}, b_{1} \mid 0\right] d z_{1} \cdots \int_{z_{k-1}}^{y}\left[a_{k}, b_{k} \mid 0\right] d z_{k} f^{\{\alpha\}}\left(p_{1}, \ldots, p_{k}\right) L_{a+b}(x, y)_{\mid b=0}
\end{aligned}
$$

with

$$
f^{\{\alpha\}}\left(p_{1}, \ldots, p_{k}\right):=\tilde{W}_{1}\left(q_{1}\right) \cdots \tilde{W}_{k}\left(q_{k}\right) e^{-i\left(q_{1} z_{1}+\cdots+q_{k} z_{k}\right)},
$$

where $p_{l}$ and $q_{l}$ are related to each other via (3.53). The explicit inverse of (3.53),

$$
q_{l}=p_{l}-\left(1-\alpha_{l}\right) p_{l+1}, \quad 1 \leq l \leq k \quad \text { and } \quad q_{k}=p_{k},
$$

shows that this transformation is regular for all values of the parameters $\alpha_{l}$. As a consequence, the function $f^{\{\alpha\}}\left(p_{1}, \ldots, p_{k}\right)$ is $C^{2}$ and has rapid decay at infinity, both uniformly in $\alpha_{l}$ (more precisely, the Schwartz norms $\left\|f^{\{\alpha\}}\right\|_{p, 2}, p \geq 0$, are all uniformly bounded in $\alpha_{l}$ ). Because of this uniformity, we need not care about the dependence of $f^{\{\alpha\}}$ on the parameters $\alpha_{l}$ in the following.

According to the bound (3.44), $r \leq k$. Thus we can insert into (3.58) the identity

$$
1=\int_{-\infty}^{\infty} d a_{1} \cdots \int_{-\infty}^{\infty} d a_{r} \delta\left(a_{1}-p_{1}^{2}\right) \cdots \delta\left(a_{r}-p_{r}^{2}\right)
$$

pull the $a_{l}$-integrals outside and carry out the integrations over $p_{1}, \ldots, p_{r}$. This gives

$$
\begin{aligned}
& \Delta P^{\mathrm{le}}(x, y)=\left(\frac{d}{d b}\right)^{r} \int_{-\infty}^{\infty} d a_{1} \cdots \int_{-\infty}^{\infty} d a_{r} \int \frac{d^{4} p_{r+1}}{(2 \pi)^{4}} \cdots \int \frac{d^{4} p_{k}}{(2 \pi)^{4}} \\
& \times \int_{x}^{y}\left[a_{1}, b_{1} \mid 0\right] d z_{1} \cdots \int_{z_{k-1}}^{y}\left[a_{k}, b_{k} \mid 0\right] d z_{k} U\left(a_{1}, \ldots, a_{r}, p_{r+1}, \ldots, p_{k}\right) L_{a+b}(x, y)_{\mid b=0}(3.5
\end{aligned}
$$

with

$$
U=\int \frac{d^{4} p_{1}}{(2 \pi)^{4}} \cdots \int \frac{d^{4} p_{r}}{(2 \pi)^{4}} \delta\left(a_{1}-p_{1}^{2}\right) \cdots \delta\left(a_{r}-p_{r}^{2}\right) f^{\{\alpha\}}\left(p_{1}, \ldots, p_{k}\right)
$$

In (3.59), the parameter $a$ depends on $a_{l}, p_{l}$, and $\alpha_{l}$ via

$$
\begin{aligned}
a= & \left(\alpha_{1}^{2}-\alpha_{1}\right) a_{1}+\cdots+\left(1-\alpha_{1}\right) \cdots\left(1-\alpha_{r-1}\right)\left(\alpha_{r}^{2}-\alpha_{r}\right) a_{r} \\
& +\left(1-\alpha_{1}\right) \cdots\left(1-\alpha_{r}\right)\left(\alpha_{r+1}^{2}-\alpha_{r+1}\right) p_{r+1}^{2} \\
& +\cdots+\left(1-\alpha_{1}\right)\left(1-\alpha_{2}\right) \cdots\left(1-\alpha_{k-1}\right)\left(\alpha_{k}^{2}-\alpha_{k}\right) p_{k}^{2}
\end{aligned}
$$

Since $f^{\{\alpha\}}$ is $C^{2}$ and has rapid decay at infinity, it follows by evaluating the integrals over the mass shells in (3.60) that $U$ is $C^{1}$ in the variables $a_{l}$ and has rapid decay at infinity.

Next, we take the Fourier transform in the variables $a$ and $a_{1}, \ldots, a_{r}$ by substituting (3.57) and

$$
\begin{aligned}
& U\left(a_{1}, \ldots, a_{r}, p_{r+1}, \ldots, p_{l}\right) \\
& \quad=\int_{-\infty}^{\infty} \frac{d \tau_{1}}{2 \pi} \cdots \int_{-\infty}^{\infty} \frac{d \tau_{r}}{2 \pi} \tilde{U}\left(\tau_{1}, \ldots, \tau_{r}, p_{r+1}, \ldots, p_{k}\right) e^{-i\left(\tau_{1} a_{1}+\cdots+\tau_{r} a_{r}\right)}
\end{aligned}
$$


into (3.59). The $a_{l}$-dependence of the resulting expression for $\Delta P^{\mathrm{le}}$ has the form of plane waves; thus the $a_{l}$-integrals give $\delta$-distributions. We can then also carry out the $\tau_{l}$-integrations. Finally, the $b$-derivatives in (3.59) give a factor $(-i \tau)^{r}$, and we obtain

$$
\begin{aligned}
\Delta P^{\mathrm{le}}(x, y)= & \int_{x}^{y}\left[a_{1}, b_{1} \mid 0\right] d z_{1} \cdots \int_{z_{k-1}}^{y}\left[a_{k}, b_{k} \mid 0\right] d z_{k} \int \frac{d^{4} p_{r+1}}{(2 \pi)^{4}} \cdots \int \frac{d^{4} p_{k}}{(2 \pi)^{4}} \\
& \times \int_{-\infty}^{\infty} \frac{d \tau}{2 \pi} \tilde{U}\left(\tau_{1}, \ldots, \tau_{r}, p_{r+1}, \ldots, p_{k}\right)(-i \tau)^{r} \tilde{L}_{\tau}(x, y)
\end{aligned}
$$

where the parameters $\tau_{l}$ are given in terms of $\tau$ and $\alpha_{l}$ by

$$
\tau_{l}=\left(1-\alpha_{1}\right) \cdots\left(1-\alpha_{l-1}\right)\left(\alpha_{l}-\alpha_{l}^{2}\right) \tau \text {. }
$$

The Fourier transform $\tilde{L}_{\tau}(x, y)$ is an integrable function in $\tau$ which depends smoothly on $x$ and $y$ (this can e.g. be verified by writing the infinite sum (3.50) with the Bessel functions $J_{1}$ and $K_{1}$ which decay at infinity). Since $U$ is $C^{2}$ in the parameters $a_{l}$ and has rapid decay, its Fourier transform $\tilde{U}$ is a function in $\tau_{l}$ which decays at infinity at least like $\mathcal{O}\left(\tau_{l}^{-1}\right)$.

We now estimate the $\alpha_{l}$-integrals in (3.61) for $l=1, \ldots, r$, from the right to the left. Since $\tilde{U}$ decays in $\tau_{r}$ at infinity like $\mathcal{O}\left(\tau_{r}^{-1}\right)$, we have the bound

$$
\int_{0}^{1} d \alpha_{r} \cdots \tilde{U}_{\mid \tau_{r}=\left(1-\alpha_{1}\right) \cdots\left(1-\alpha_{r-1}\right)\left(\alpha_{r}-\alpha_{r}^{2}\right) \tau} \leq \sup _{\alpha_{r} \in[0,1]} \cdots\left(\left(1-\alpha_{1}\right) \cdots\left(1-\alpha_{r-1}\right) \tau\right)^{-1} g,
$$

where we have for clarity only written out the $\alpha_{r}$-integral; $g$ is a function depending on the variables $\tau_{1}, \ldots, \tau_{r-1}$ and $p_{r+1}, \ldots, p_{k}$. The inequality (3.45) implies that all factors $(1-$ $\left.\alpha_{l}\right)^{-1}$ cancel against corresponding factors $\left(1-\alpha_{l}\right)$ in the nested line integrals. The decay properties in the remaining parameters $\tau_{1}, \ldots, \tau_{r-1}$ remain unchanged by our estimate of the $\alpha_{r}$-integral. Therefore we can proceed inductively in the same way for the integrals over $\alpha_{r-1}, \alpha_{r-2}, \ldots, \alpha_{1}$. The bounds (3.45) ensure that all factors $\left(1-\alpha_{l}\right)^{-1}$ drop out. Since we get a factors $\tau^{-1}$ in each step, the factor $(-i \tau)^{r}$ in (3.61) disappears. Using that $\tilde{L}_{\tau}$ is integrable and that we have fast decay at infinity in the variables $p_{r+1}, \ldots, p_{k}$, all the remaining integrals are finite. We conclude that $\Delta P^{\mathrm{le}}(x, y)$ is well-defined.

If we take partial derivatives of (3.56) with respect to $x$ and $y$, the derivatives act either on the exponentials, yielding additional factors $\alpha_{l},\left(1-\alpha_{l}\right)$, and $q_{l}$, or they act on $L_{a}(x, y)$. Since $L_{a}$ and its Fourier transform $\tilde{L}_{\tau}$ depend smoothly on $x$ and $y$, we can repeat the above mollifying argument and conclude that $\Delta P^{\mathrm{le}}(x, y)$ is smooth.

For a very rigid mathematician, it might not seem quite satisfying that the light-cone expansion of the residual fermionic projector was first performed on a formal level and later made rigorous by resumming the formal expansion. We remark that one could avoid all formal series in intermediate steps of the construction by already "regularizing" the logarithmic mass terms in the Green's functions $s^{ \pm}$after (3.12). However, this has the disadvantage of becoming quite technical. Our procedure is easier to understand because we could introduce the residual argument in Subsection 3.1 without entering the mathematical details right away.

Similar to the high energy contribution, the low energy contribution $\tilde{P}^{l e}(x, y)$ is noncausal in the sense that it depends on the external potential in the entire Minkowski space. This can be understood from the fact that the operator products in the perturbation expansion (3.16) contain factors $p\left(z_{1}, z_{2}\right)$, whose support is the whole space $\left(z_{1}, z_{2}\right) \in$ 
$\mathbb{R}^{4} \times \mathbb{R}^{4}$. We point out that, although the statements of Theorem 3.4 and Theorem 3.8 are very similar, their proofs are completely different. This shows and illustrates that the high and low energy contributions describe two different physical effects. In contrast to the high energy contribution, the potential in the low energy contribution need not overcome an "energy gap;" as a consequence, the low energy contribution plays an important role even if the energy of the external potential is small.

This concludes our analysis of the Dirac sea. We briefly summarize our main results: According to Def. 3.1, Def. 3.3, and Def. 3.5, we decompose the fermionic projector in the form

$$
\tilde{P}(x, y)=\tilde{P}^{\text {causal }}(x, y)+\tilde{P}^{\text {le }}(x, y)+\tilde{P}^{\text {he }}(x, y) .
$$

The causal contribution $\tilde{P}^{\text {causal }}(x, y)$ has singularities on the light cone, which are completely described by the light-cone expansion of Theorem 3.6. The non-causal low and high energy contributions $\tilde{P}^{\text {le }}(x, y)$ and $\tilde{P}^{\text {he }}(x, y)$, on the other hand, are, to every order in perturbation theory, smooth functions in $x$ and $y$ (see Theorem 3.4 and Theorem 3.8).

We finally point out that, in contrast to $\tilde{P}^{\text {causal }}$, both non-causal contributions $\vec{P}^{\text {le }}$ and $\tilde{P}^{\text {he }}$ were only studied to every order in perturbation theory, but we did not consider the convergence of their perturbation series. For $\tilde{P}^{\text {le }}$, this convergence problem could be studied by resumming all phase-free contributions to the formal light-cone expansion of Proposition 3.2. However, there seems to be no easy method at the moment to control the convergence of the perturbation expansion of the high energy contribution $\tilde{P}^{\text {he }}(x, y)$. Nevertheless, Theorem 3.4 and Theorem 3.8 give a strong indication that the non-causal contribution is a smooth function. Even if singularities or divergences occurred when carrying out the sum over the perturbation series (which seems unlikely), these singularities would be of very different nature than the singularities of $\tilde{P}^{\text {causal }}(x, y)$. More precisely, they could not be expressed in terms of the external potential and its derivatives along the convex line $\overline{x y}$, because singularities of this type necessarily show up in finite order perturbation theory. Thus we can say that, although the non-causal contributions $\tilde{P}^{l e}$ and $\tilde{P}^{\text {he }}$ require further study in order to get a complete understanding, the decomposition (3.62) is well-established. The light-cone expansion gives a method to explicitly calculate the causal contribution $\tilde{P}^{\text {causal }}(x, y)$.

\section{A Some Formulas of the Light-Cone Expansion}

In this appendix, we give a compilation of explicit formulas of the light-cone expansion. More precisely, we list the phase-free contribution to the light-cone expansion of the Green's functions (cf. Def. 2.7). According to the reduction to the phase-free contribution, the light-cone expansion of the Green's functions is immediately obtained by inserting ordered exponentials into the line integrals, see Def. 2.9 and Theorem 2.10. Furthermore, using Theorem 3.6, the formulas can be directly applied to the fermionic projector; they then describe the singularities of $\tilde{P}(x, y)$ on the light cone.

All the following formulas were generated by the $\mathrm{C}++$ program "class_commute" (see Subsection 2.3). Our listings are not intended to be in any sense complete; we made a selection in order to give the reader a first impression of the form of the singularities. The more detailed formulas to higher order on the light cone can be easily obtained with "class_commute." Without loss of generality, we restrict ourselves to the left handed component of the Green's functions; for the right handed component, the formulas are analogous. 
We begin with the perturbation by a chiral perturbation to first order. The phase-free contribution to the order $\mathcal{O}\left((y-x)^{2}\right)$ on the light cone is

$$
\begin{aligned}
\chi_{L} & \left(-s\left(\chi_{L} A_{R}+\chi_{R} A_{L}\right) s\right)(x, y) \stackrel{\text { phase-free }}{\asymp} \mathcal{O}\left((y-x)^{2}\right) \\
& +\chi_{L} S^{(0)}(x, y) \xi^{i} \int_{x}^{y} d z[0,1 \mid 0]\left(\not \partial A_{L i}\right) \\
& -\chi_{L} S^{(0)}(x, y) \int_{x}^{y} d z[0,0 \mid 0] A_{L} \\
& +\chi_{L} S^{(0)}(x, y) A_{L}(x) \\
& +\frac{1}{2} \chi_{L} S^{(0)}(x, y) \not \int_{x}^{y} d z[0,0 \mid 0]\left(\not \partial A_{L}\right) \\
& -\chi_{L} S^{(0)}(x, y) \not \int_{x}^{y} d z[1,0 \mid 0]\left(\partial^{i} A_{L i}\right) \\
& +\frac{1}{2} \chi_{L} S^{(0)}(x, y) \not \xi^{i} \int_{x}^{y} d z[0,0 \mid 1]\left(\square A_{L i}\right) \\
& +\chi_{L} S^{(1)}(x, y) \xi^{i} \int_{x}^{y} d z[0,1 \mid 1]\left(\not \partial \square A_{L i}\right) \\
& +\chi_{L} S^{(1)}(x, y) \int_{x}^{y} d z[0,2 \mid 0]\left(\square A_{L}\right) \\
& -2 \chi_{L} S^{(1)}(x, y) \int_{x}^{y} d z[0,0 \mid 1]\left(\not \partial \partial^{i} A_{L i}\right) \\
& +\frac{1}{2} \chi_{L} S^{(1)}(x, y) \not \xi \int_{x}^{y} d z[0,0 \mid 1]\left(\not \partial \square A_{L}\right) \\
& -\chi_{L} S^{(1)}(x, y) \not \& \int_{x}^{y} d z[1,0 \mid 1]\left(\partial^{i} \square A_{L i}\right) \\
& +\frac{1}{4} \chi_{L} S^{(1)}(x, y) \not \xi \xi^{i} \int_{x}^{y} d z[0,0 \mid 2]\left(\square^{2} A_{L i}\right)
\end{aligned}
$$

where we used the abbreviation $\xi \equiv(y-x)$. This formula has the disadvantage that it contains ordinary partial derivatives of the chiral potential; it would be better for physical applications to work instead with the Yang-Mills field tensor and the Yang-Mills current. Therefore, we introduce left and right handed gauge-covariant derivatives $D^{L / R}$,

$$
D_{j}^{L}=\frac{\partial}{\partial x^{j}}-i A_{L j}, \quad D_{j}^{R}=\frac{\partial}{\partial x^{j}}-i A_{R j},
$$

and define the corresponding field tensor and current as usual by the commutators

$$
F_{j k}^{c}=i\left[D_{j}^{c}, D_{k}^{c}\right], \quad j_{l}^{c}=\left[D^{c k}, F_{l k}^{c}\right] \quad(c=L \text { or } R) .
$$

In the Abelian case of a single Dirac sea (i.e. $f=1$ ), (A.13) reduces to the familiar formulas for the electromagnetic field tensor and current,

$$
F_{j k}^{c}=\partial_{j} A_{c k}-\partial_{k} A_{c j} \quad, \quad j_{l}^{c}=\partial_{l k} A_{c}^{k}-\square A_{c l}
$$

Notice, however, that in the general case of a system of Dirac seas, (A.13) involves quadratic and cubic terms in the potential.

By substituting (A.13) into (A.1) $-($ A.12 $)$ and manipulating the line integrals with partial integrations, one can rewrite the phase-free contribution in a way where the linear 
terms in the potential are gauge invariant. For example, we can combine (A.1), (A.2), and (A.3) by transforming the line integrals as

$$
\begin{gathered}
\xi^{k} \int_{x}^{y} d z[0,1 \mid 0]\left(\not \partial A_{L k}\right)=\xi^{k} \int_{x}^{y} d z[0,1 \mid 0]\left(\gamma^{j} F_{j k}^{L}+\partial_{k} A_{L}\right)+\mathcal{O}\left(A_{L}^{2}\right) \\
=\xi^{k} \int_{x}^{y} d z[0,1 \mid 0] \gamma^{j} F_{j k}^{L}-A_{L}(x)+\int_{x}^{y} d z[0,0 \mid 0] A_{L}+\mathcal{O}\left(A_{L}^{2}\right)
\end{gathered}
$$

However, this procedure yields (in the non-Abelian case) quadratic and cubic terms in the potential which are not gauge invariant. Fortunately, these gauge-dependent terms are all compensated by corresponding contributions of the higher order Feynman diagrams. More generally, it turns out that, after summing up the perturbation series for the chiral perturbation, we can arrange a gauge invariant phase-free contribution for which the insertion rules of Def. 2.9 and the statement of Theorem 2.10 hold. This is not astonishing in view of the behavior (2.102) of the Green's functions under local gauge transformations; we verified it explicitly term by term for all following formulas. We now list the gauge invariant phase-free contribution to the order $\mathcal{O}\left((y-x)^{2}\right)$ on the light cone. For simplicity, we omit all third order terms in the potential which are of the order $\mathcal{O}\left((y-x)^{0}\right)$ on the light cone and which have a prefactor $\&$ (the combinatorics of the tensor contractions leads to many such terms, but they are not very instructive here).

$$
\begin{aligned}
& \chi_{L} \sum_{k=0}^{\infty}\left(\left(-s\left(\chi_{L} A_{R}+\chi_{R} A_{L}\right)\right)^{k} s\right)(x, y) \\
& \check{\text { phase-free }}^{\mathcal{O}} \mathcal{O}\left((y-x)^{2}\right)+\not \& A_{L}^{i} A_{L}^{j} A_{L}^{k} \mathcal{O}\left((y-x)^{0}\right) \\
& +\chi_{L} S^{(0)}(x, y) \xi^{i} \int_{x}^{y} d z[0,1 \mid 0] \gamma^{l} F_{l i}^{L} \\
& +\frac{1}{4} \chi_{L} S^{(0)}(x, y) \& \int_{x}^{y} d z[0,0 \mid 0] \gamma^{j} \gamma^{k} F_{j k}^{L} \\
& -\frac{1}{2} \chi_{L} S^{(0)}(x, y) \not \xi^{i} \int_{x}^{y} d z[0,0 \mid 1] j_{i}^{L} \\
& +\chi_{L} S^{(1)}(x, y) \xi^{i} \int_{x}^{y} d z[0,1 \mid 1]\left(\not \partial j_{i}^{L}\right) \\
& +\chi_{L} S^{(1)}(x, y) \int_{x}^{y} d z[0,2 \mid 0] j_{k}^{L} \gamma^{k} \\
& -\frac{1}{2} \chi_{L} S^{(1)}(x, y) \not \int_{x}^{y} d z[0,0 \mid 1]\left(\not \partial j_{k}^{L}\right) \gamma^{k} \\
& -\frac{1}{4} \chi_{L} S^{(1)}(x, y) \& \xi^{i} \int_{x}^{y} d z[0,0 \mid 2]\left(\square j_{i}^{L}\right) \\
& -i \chi_{L} S^{(0)}(x, y) \not \& \xi_{i} \xi^{j} \int_{x}^{y} d z_{1}[0,1 \mid 1] F_{k j}^{L} \int_{z_{1}}^{y} d z_{2}[0,1 \mid 0] F_{L}^{k i} \\
& +i \chi_{L} S^{(1)}(x, y) \xi^{i} \xi^{j} \int_{x}^{y} d z_{1}[0,3 \mid 0] \gamma^{k} F_{k j}^{L} \int_{z_{1}}^{y} d z_{2}[0,0 \mid 1] j_{i}^{L} \\
& +i \chi_{L} S^{(1)}(x, y) \xi^{i} \xi^{j} \int_{x}^{y} d z_{1}[0,2 \mid 1] j_{j}^{L} \int_{z_{1}}^{y} d z_{2}[0,1 \mid 0] \gamma^{l} F_{l i}^{L} \\
& -2 i \chi_{L} S^{(1)}(x, y) \xi_{i} \xi^{j} \int_{x}^{y} d z_{1}[0,2 \mid 1] F_{m j}^{L} \int_{z_{1}}^{y} d z_{2}[0,2 \mid 0]\left(\not \partial F_{L}^{m i}\right) \\
& -2 i \chi_{L} S^{(1)}(x, y) \xi_{i} \xi^{j} \int_{x}^{y} d z_{1}[0,2 \mid 1]\left(\not \partial F_{k j}^{L}\right) \int_{z_{1}}^{y} d z_{2}[0,1 \mid 0] F_{L}^{k i}
\end{aligned}
$$




$$
\begin{aligned}
& +i \chi_{L} S^{(1)}(x, y) \xi^{i} \xi^{j} \int_{x}^{y} d z_{1}[0,2 \mid 1] \gamma^{k} F_{k j}^{L} \int_{z_{1}}^{y} d z_{2}[0,2 \mid 0] j_{i}^{L} \\
& -\frac{i}{2} \chi_{L} S^{(1)}(x, y) \xi^{i} \int_{x}^{y} d z_{1}[0,2 \mid 0] \gamma^{j} F_{j i}^{L} \int_{z_{1}}^{y} d z_{2}[0,0 \mid 0] \gamma^{k} \gamma^{l} F_{k l}^{L} \\
& -\frac{i}{2} \chi_{L} S^{(1)}(x, y) \xi^{i} \int_{x}^{y} d z_{1}[0,2 \mid 0] \gamma^{j} \gamma^{k} F_{j k}^{L} \int_{z_{1}}^{y} d z_{2}[0,1 \mid 0] \gamma^{l} F_{l i}^{L} \\
& +2 i \chi_{L} S^{(1)}(x, y) \xi_{i} \int_{x}^{y} d z_{1}[0,3 \mid 0] \gamma^{j} F_{j k}^{L} \int_{z_{1}}^{y} d z_{2}[0,1 \mid 0] F_{L}^{k i} \\
& -2 i \chi_{L} S^{(1)}(x, y) \xi^{j} \int_{x}^{y} d z_{1}[0,1 \mid 1] F_{i j}^{L} \int_{z_{1}}^{y} d z_{2}[0,1 \mid 0] \gamma_{k} F_{L}^{k i} \\
& -\frac{i}{2} \chi_{L} S^{(1)}(x, y) \not \xi^{j} \xi^{k} \int_{x}^{y} d z_{1}[0,2 \mid 1] j_{j}^{L} \int_{z_{1}}^{y} d z_{2}[0,0 \mid 1] j_{k}^{L} \\
& -\frac{i}{2} \chi_{L} S^{(1)}(x, y) \not \xi^{j} \xi^{k} \int_{x}^{y} d z_{1}[0,1 \mid 2] j_{j}^{L} \int_{z_{1}}^{y} d z_{2}[0,2 \mid 0] j_{k}^{L} \\
& +i \chi_{L} S^{(1)}(x, y) \not \xi \xi^{i} \xi^{j} \int_{x}^{y} d z_{1}[0,2 \mid 1] F_{k j}^{L} \int_{z_{1}}^{y} d z_{2}[0,1 \mid 1]\left(\partial^{k} j_{i}^{L}\right) \\
& +i \chi_{L} S^{(1)}(x, y) \not \xi \xi^{i} \xi^{j} \int_{x}^{y} d z_{1}[0,1 \mid 2] F_{k j}^{L} \int_{z_{1}}^{y} d z_{2}[0,3 \mid 0]\left(\partial^{k} j_{i}^{L}\right) \\
& -i \chi_{L} S^{(1)}(x, y) \not \xi \xi_{i} \xi^{j} \int_{x}^{y} d z_{1}[0,1 \mid 2]\left(\partial_{k} F_{l j}^{L}\right) \int_{z_{1}}^{y} d z_{2}[0,2 \mid 0]\left(\partial^{k} F_{L}^{l i}\right) \\
& +\frac{i}{4} \chi_{L} S^{(1)}(x, y) \not \xi^{i} \int_{x}^{y} d z_{1}[0,2 \mid 0] \gamma^{j} \gamma^{k} F_{j k}^{L} \int_{z_{1}}^{y} d z_{2}[0,0 \mid 1] j_{i}^{L} \\
& +\frac{i}{4} \chi_{L} S^{(1)}(x, y) \& \xi^{i} \int_{x}^{y} d z_{1}[0,1 \mid 1] \gamma^{j} \gamma^{k} F_{j k}^{L} \int_{z_{1}}^{y} d z_{2}[0,2 \mid 0] j_{i}^{L} \\
& +\frac{i}{4} \chi_{L} S^{(1)}(x, y) \& \xi^{i} \int_{x}^{y} d z_{1}[0,1 \mid 1] j_{i}^{L} \int_{z_{1}}^{y} d z_{2}[0,0 \mid 0] \gamma^{j} \gamma^{k} F_{j k}^{L} \\
& -\frac{i}{2} \chi_{L} S^{(1)}(x, y) \not \xi \xi^{i} \int_{x}^{y} d z_{1}[0,1 \mid 1] \gamma^{j} \gamma^{k}\left(\partial^{l} F_{j k}^{L}\right) \int_{z_{1}}^{y} d z_{2}[0,1 \mid 0] F_{l i}^{L} \\
& -\frac{i}{2} \chi_{L} S^{(1)}(x, y) \& \xi^{i} \int_{x}^{y} d z_{1}[0,1 \mid 1] F_{j i}^{L} \int_{z_{1}}^{y} d z_{2}[0,1 \mid 0] \gamma^{k} \gamma^{l}\left(\partial^{j} F_{k l}^{L}\right) \\
& +i \chi_{L} S^{(1)}(x, y) \not \xi^{i} \xi^{j} \int_{x}^{y} d z_{1}[0,1 \mid 2]\left(\partial^{k} j_{j}^{L}\right) \int_{z_{1}}^{y} d z_{2}[0,1 \mid 0] F_{k i}^{L} \\
& -i \chi_{L} S^{(1)}(x, y) \& \xi^{j} \int_{x}^{y} d z_{1}[0,1 \mid 1] F_{i j}^{L} \int_{z_{1}}^{y} d z_{2}[0,0 \mid 1] j_{L}^{i} \\
& -i \chi_{L} S^{(1)}(x, y) \not \& \xi^{j} \int_{x}^{y} d z_{1}[0,0 \mid 2] F_{i j}^{L} \int_{z_{1}}^{y} d z_{2}[0,2 \mid 0] j_{L}^{i} \\
& +i \chi_{L} S^{(1)}(x, y) \not \xi^{i} \int_{x}^{y} d z_{1}[0,1 \mid 1] j_{L}^{j} \int_{z_{1}}^{y} d z_{2}[0,1 \mid 0] F_{j i}^{L} \\
& +2 i \chi_{L} S^{(1)}(x, y) \& \xi_{i} \int_{x}^{y} d z_{1}[0,2 \mid 1] F_{k l}^{L} \int_{z_{1}}^{y} d z_{2}[0,2 \mid 0]\left(\partial^{k} F_{L}^{l i}\right) \\
& -2 i \chi_{L} S^{(1)}(x, y) \& \xi^{j} \int_{x}^{y} d z_{1}[0,0 \mid 2]\left(\partial_{k} F_{l j}^{L}\right) \int_{z_{1}}^{y} d z_{2}[0,1 \mid 0] F_{L}^{k l} \\
& -\frac{i}{8} \chi_{L} S^{(1)}(x, y) \not \int_{x}^{y} d z_{1}[0,1 \mid 0] \gamma^{j} \gamma^{k} F_{j k}^{L} \int_{z_{1}}^{y} d z_{2}[0,0 \mid 0] \gamma^{l} \gamma^{m} F_{l m}^{L} \\
& +3 i \chi_{L} S^{(1)}(x, y) \not \& \int_{x}^{y} d z_{1}[0,1 \mid 1] F_{k l}^{L} \int_{z_{1}}^{y} d z_{2}[0,1 \mid 0] F_{L}^{k l}
\end{aligned}
$$




$$
\begin{aligned}
& -2 \chi_{L} S^{(1)}(x, y) \xi_{i} \xi^{j} \xi^{k} \int_{x}^{y} d z_{1}[0,4 \mid 0] \gamma^{l} F_{l k}^{L} \int_{z_{1}}^{y} d z_{2}[0,1 \mid 1] F_{m j}^{L} \int_{z_{2}}^{y} d z_{3}[0,1 \mid 0] F_{L}^{m i} \\
& -2 \chi_{L} S^{(1)}(x, y) \xi_{i} \xi^{j} \xi^{k} \int_{x}^{y} d z_{1}[0,3 \mid 1] \gamma^{l} F_{l k}^{L} \int_{z_{1}}^{y} d z_{2}[0,3 \mid 0] F_{m j}^{L} \int_{z_{2}}^{y} d z_{3}[0,1 \mid 0] F_{L}^{m i} \\
& -2 \chi_{L} S^{(1)}(x, y) \xi_{i} \xi^{j} \xi^{k} \int_{x}^{y} d z_{1}[0,3 \mid 1] F_{m k}^{L} \int_{z_{1}}^{y} d z_{2}[0,3 \mid 0] \gamma^{l} F_{l j}^{L} \int_{z_{2}}^{y} d z_{3}[0,1 \mid 0] F_{L}^{m i} \\
& -2 \chi_{L} S^{(1)}(x, y) \xi^{i} \xi_{j} \xi^{k} \int_{x}^{y} d z_{1}[0,3 \mid 1] F_{m k}^{L} \int_{z_{1}}^{y} d z_{2}[0,3 \mid 0] F_{L}^{m j} \int_{z_{2}}^{y} d z_{3}[0,1 \mid 0] \gamma^{l} F_{l i}^{L}
\end{aligned}
$$

We call this formulation of the phase-free contributions purely in terms of the YangMills field tensor and the Yang-Mills current the gauge invariant form of the light-cone expansion.

For clarity, we mention a subtlety in the transformation to the gauge invariant form: The gauge invariant phase-free contribution implicitly contains tangential derivatives of the chiral potential (as one sees by writing out $F^{c}$ and $j^{c}$ in in terms of the chiral potentials). Thus it is not a phase-free contribution in correspondence with Def. 2.7; as a consequence, it is not obvious that the statement of Theorem 2.10 holds. In other words, one must be very careful when transforming the line integrals in order to ensure that the insertion rules of Def. 2.9 and Theorem 2.10 remain valid. A safe method is to insert all ordered exponentials into the line integrals before performing the partial integrations. According to Theorem 2.10, the phase-inserted formulas coincide with the light-cone expansion of the Green's functions. Therefore the partial integrations become identical transformations of the Green's functions; we need not worry about the insertion rules. In the final step, we again take out the ordered exponentials from the line integrals, verifying that they are still in accordance with Def. 2.9. In the example of the line integral (A.14), the partial integration of the phase-inserted contribution gives

$$
\begin{aligned}
\xi^{k} \int_{x}^{y} d z[0,1 \mid 0] \mathrm{Pe}^{-i \int_{x}^{z} A_{L}^{l}(z-x)_{l}} \not \partial A_{L k}(z) \mathrm{Pe}^{-i \int_{z}^{y} A_{L}^{m}(y-z)_{m}} \\
=\xi^{k} \int_{x}^{y} d z[0,1 \mid 0] \mathrm{Pe}^{-i \int_{x}^{z} A_{L}^{l}(z-x)_{l}}\left(\gamma^{j} F_{j k}^{L}+i\left[A_{L}, A_{L k}\right]+\partial_{k} A_{L}\right)_{\mid z} \mathrm{Pe}^{-i \int_{z}^{y} A_{L}^{m}(y-z)_{m}} \\
=\xi^{k} \int_{x}^{y} d z[0,1 \mid 0] \mathrm{Pe}^{-i \int_{x}^{z} A_{L}^{l}(z-x)_{l}}\left(\gamma^{j} F_{j k}^{L}+i\left[A_{L}, A_{L k}\right]\right)_{\mid z} \mathrm{Pe}^{-i \int_{z}^{y} A_{L}^{m}(y-z)_{m}} \text { (A.15) } \\
\quad-A_{L}(x) \mathrm{Pe}^{-i \int_{x}^{y} A_{L}^{m}(y-z)_{x}} \\
\quad+\int_{x}^{y} d z[0,0 \mid 0] \mathrm{Pe}^{-i \int_{x}^{z} A_{L}^{l}(z-x)_{l}} A_{L}(z) \mathrm{Pe}^{-i \int_{z}^{y} A_{L}^{m}(y-z)_{m}} \\
\quad+\xi^{k} \int_{x}^{y} d z[0,1 \mid 0] \mathrm{Pe}^{-i \int_{x}^{z} A_{L}^{l}(z-x)_{l}} i\left[A_{L k}, A_{L}\right](z) \mathrm{Pe}^{-i \int_{z}^{y} A_{L}^{m}(y-z)_{m}}
\end{aligned}
$$

Thus the correct transformation of the phase-free contribution is

$\xi^{k} \int_{x}^{y} d z[0,1 \mid 0]\left(\not \partial A_{L k}\right) \stackrel{\text { phase-free }}{\simeq} \xi^{k} \int_{x}^{y} d z[0,1 \mid 0] \gamma^{j} F_{j k}^{L}+\int_{x}^{y} d z[0,0 \mid 0] A_{L}-A_{L}(x)$.

Notice that (A.18) are the derivative terms of the ordered exponentials; they get lost if the phase-free contribution is transformed in a naive way.

It remains to consider the scalar/pseudoscalar perturbation; i.e., we must study how the dynamic mass matrices $Y_{L / R}(x)$ show up in the light-cone expansion. We begin with the case of a single mass matrix. To first order in the external potential, the corresponding 
Feynman diagram has the light-cone expansion

$$
\begin{array}{rl}
\chi_{L} & m\left(-s\left(-\chi_{L} Y_{R}-\chi_{R} Y_{L}\right) s\right)(x, y) \stackrel{\text { phase-free }}{\asymp} \mathcal{O}\left((y-x)^{2}\right) \\
& +\frac{1}{2} \chi_{L} m S^{(0)}(x, y) \not \int_{x}^{y} d z[0,0 \mid 0]\left(\not \partial Y_{L}\right) \\
& +\chi_{L} m S^{(0)}(x, y) Y_{L}(x) \\
& +\chi_{L} m S^{(1)}(x, y) \int_{x}^{y} d z[0,1 \mid 0]\left(\square Y_{L}\right) \\
& +\frac{1}{2} \chi_{L} m S^{(1)}(x, y) \not \int_{x}^{y} d z[0,0 \mid 1]\left(\not \supset \square Y_{L}\right) .
\end{array}
$$

Similar to (A.1)-(A.12), this formula involves partial derivatives of the potential, which is not a gauge invariant formulation. Since the chirality changes at every mass matrix (see e.g. Def. 2.9), the correct way to make the light-cone expansion gauge invariant is to work with the gauge-covariant mass derivatives $D, \Delta$ given by

$$
\begin{aligned}
\left(D_{i} Y_{L}\right) & =D_{i}^{L} Y_{L}-Y_{L} D_{i}^{R}=\left(\partial_{i} Y_{L}\right)-i A_{L i} Y_{L}+i Y_{L} A_{R i} \\
\left(\Delta Y_{L}\right) & =D_{i}\left(D^{i} Y_{L}\right)=D_{i}^{L}\left(D^{i} Y_{L}\right)-\left(D^{i} Y_{L}\right) D_{i}^{R}
\end{aligned}
$$

and similarly for $Y_{R}$ and higher derivatives. Rewriting (A.19) with gauge-covariant mass derivatives yields additional terms which are linear or quadratic in the chiral potentials and which are not gauge invariant. But, similar as described for the chiral perturbation above, all these terms cancel if the sum over the perturbation series for the chiral potentials is carried out. To the order $\mathcal{O}\left((y-x)^{2}\right)$ on the light cone, we obtain in this way the gauge invariant phase-free contribution

$$
\begin{aligned}
\chi_{L} m & \sum_{n_{1}, n_{2}=0}^{\infty}\left(\left(-s\left(\chi_{L} A_{R}+\chi_{R} A_{L}\right)\right)^{n_{1}} s\left(\chi_{L} Y_{R}+\chi_{R} Y_{L}\right) s\left(\left(-\chi_{L} A_{R}-\chi_{R} A_{L}\right) s\right)^{n_{2}}\right)(x, y) \\
= & \chi_{L} \sum_{n_{1}, n_{2}=0}^{\infty}\left(\left(-s A_{L}\right)^{n_{1}} s Y_{L} s\left(-A_{R} s\right)^{n_{2}}\right)(x, y) \stackrel{\text { phase-free }}{=}\left((y-x)^{2}\right) \\
& +\frac{1}{2} \chi_{L} m S^{(0)}(x, y) \not \int_{x}^{y} d z[0,0 \mid 0] \gamma^{j}\left(D_{j} Y_{L}\right) \\
& +\chi_{L} m S^{(0)}(x, y) Y_{L}(x) \\
& +\chi_{L} m S^{(1)}(x, y) \int_{x}^{y} d z[0,1 \mid 0]\left(\Delta Y_{L}\right) \\
& +\frac{1}{2} \chi_{L} m S^{(1)}(x, y) \not \int_{x}^{y} d z[0,0 \mid 1] \gamma^{j}\left(D_{j} \Delta Y_{L}\right) \\
& +\frac{i}{2} \chi_{L} m S^{(1)}(x, y) \not \xi \xi^{i} \int_{x}^{y} d z_{1}[0,1 \mid 1] j_{i}^{L} \int_{z_{1}}^{y} d z_{2}[0,0 \mid 0] \gamma^{j}\left(D_{j} Y_{L}\right) \\
& -i \chi_{L} m S^{(1)}(x, y) \not \xi \xi^{i} \int_{x}^{y} d z_{1}[0,1 \mid 1] F_{j i}^{L} \int_{z_{1}}^{y} d z_{2}[0,1 \mid 0] \gamma_{k}\left(D^{k j} Y_{L}\right) \\
& -\frac{i}{4} \chi_{L} m S^{(1)}(x, y) \not \int_{x}^{y} d z_{1}[0,1 \mid 0] \gamma^{k} \gamma^{l} F_{k l}^{L} \int_{z_{1}}^{y} d z_{2}[0,0 \mid 0] \gamma^{j}\left(D_{j} Y_{L}\right) \\
& -i \chi_{L} m S^{(1)}(x, y) \xi^{i} \int_{x}^{y} d z_{1}[0,2 \mid 0] \gamma^{k} F_{k i}^{L} \int_{z_{1}}^{y} d z_{2}[0,0 \mid 0] \gamma^{j}\left(D_{j} Y_{L}\right) \\
& -\frac{i}{2} \chi_{L} m S^{(1)}(x, y) \int_{x}^{y} d z[0,1 \mid 0] \gamma^{i} \gamma^{j} F_{i j}^{L} Y_{L}
\end{aligned}
$$




$$
\begin{aligned}
& +\frac{i}{2} \chi_{L} m S^{(1)}(x, y) \not \int_{x}^{y} d z[0,0 \mid 1] j_{k}^{L} \gamma^{k} Y_{L} \\
& -i \chi_{L} m S^{(1)}(x, y) \& \int_{x}^{y} d z[0,0 \mid 1] \gamma^{j} F_{i j}^{L}\left(D^{i} Y_{L}\right) \\
& +\frac{i}{2} \chi_{L} m S^{(1)}(x, y) \not \xi \xi^{i} \int_{x}^{y} d z_{1}[0,2 \mid 0] \gamma^{j}\left(D_{j} Y_{L}\right) \int_{z_{1}}^{y} d z_{2}[0,0 \mid 1] j_{i}^{R} \\
& +\frac{i}{2} \chi_{L} m S^{(1)}(x, y) \not \xi \xi^{i} \int_{x}^{y} d z_{1}[0,1 \mid 1] \gamma^{j}\left(D_{j} Y_{L}\right) \int_{z_{1}}^{y} d z_{2}[0,2 \mid 0] j_{i}^{R} \\
& -i \chi_{L} m S^{(1)}(x, y) \& \xi^{i} \int_{x}^{y} d z_{1}[0,1 \mid 1] \gamma_{k}\left(D^{k j} Y_{L}\right) \int_{z_{1}}^{y} d z_{2}[0,1 \mid 0] F_{j i}^{R} \\
& -1 \frac{i}{4} \chi_{L} m S^{(1)}(x, y) \& \int_{x}^{y} d z_{1}[0,1 \mid 0] \gamma^{j}\left(D_{j} Y_{L}\right) \int_{z_{1}}^{y} d z_{2}[0,0 \mid 0] \gamma^{k} \gamma^{l} F_{k l}^{R} \\
& +i \chi_{L} m S^{(1)}(x, y) \xi^{i} \int_{x}^{y} d z_{1}[0,2 \mid 0] \gamma^{j}\left(D_{j} Y_{L}\right) \int_{z_{1}}^{y} d z_{2}[0,1 \mid 0] \gamma^{k} F_{k i}^{R} \\
& -2 i \chi_{L} m S^{(1)}(x, y) \xi^{i} \int_{x}^{y} d z_{1}[0,2 \mid 0]\left(D^{j} Y_{L}\right) \int_{z_{1}}^{y} d z_{2}[0,1 \mid 0] F_{j i}^{R} \\
& +i \chi_{L} m S^{(1)}(x, y) \& \int_{x}^{y} d z[0,0 \mid 1]\left(D^{j} Y_{L}\right) \gamma^{i} F_{j i}^{R} \\
& -\frac{i}{2} \chi_{L} m S^{(1)}(x, y) \not \int_{x}^{y} d z[0,0 \mid 1] Y_{L} j_{k}^{R} \gamma^{k} \\
& +i \chi_{L} m S^{(1)}(x, y) \xi^{i} Y_{L} \int_{x}^{y} d z[0,0 \mid 1] j_{i}^{R} \\
& +\frac{i}{2} \chi_{L} m S^{(1)}(x, y) \int_{x}^{y} d z[0,1 \mid 0] Y_{L} \gamma^{j} \gamma^{k} F_{j k}^{R} \\
& -\frac{i}{2} \chi_{L} m S^{(1)}(x, y) Y_{L} \int_{x}^{y} d z[0,0 \mid 0] \gamma^{j} \gamma^{k} F_{j k}^{R} \\
& +\chi_{L} m S^{(1)}(x, y) \& \xi_{i} \xi^{j} \int_{x}^{y} d z_{1}[0,2 \mid 1] F_{k j}^{L} \int_{z_{1}}^{y} d z_{2}[0,2 \mid 0] F_{L}^{k i} \int_{z_{2}}^{y}[0,0 \mid 0] \gamma^{l}\left(D_{l} Y_{L}\right) \\
& +\chi_{L} m S^{(1)}(x, y) \not \xi \xi^{j} \int_{x}^{y} d z_{1}[0,1 \mid 1] F_{m j}^{L} \int_{z_{1}}^{y} d z_{2}[0,1 \mid 0] \gamma_{k} F_{L}^{m k} Y_{L} \\
& +\chi_{L} m S^{(1)}(x, y) \& \xi_{i} \xi^{j} \int_{x}^{y} d z_{1}[0,2 \mid 1] F_{k j}^{L} \int_{z_{1}}^{y} d z_{2}[0,2 \mid 0] \gamma^{l}\left(D_{l} Y_{L}\right) \int_{z_{2}}^{y}[0,1 \mid 0] F_{R}^{k i} \\
& +\chi_{L} m S^{(1)}(x, y) \& \xi^{i} \int_{x}^{y} d z_{1}[0,1 \mid 1] F_{k i}^{L} \int_{z_{1}}^{y} d z_{2}[0,1 \mid 0] Y_{L} \gamma_{j} F_{R}^{k j} \\
& +\chi_{L} m S^{(1)}(x, y) \& \xi_{i} \int_{x}^{y} d z_{1}[0,1 \mid 1] \gamma^{j} F_{k j}^{L} Y_{L} \int_{z_{1}}^{y} d z_{2}[0,1 \mid 0] F_{R}^{k i} \\
& +\chi_{L} m S^{(1)}(x, y) \& \xi_{i} \xi^{j} \int_{x}^{y} d z_{1}[0,3 \mid 0] \gamma^{l}\left(D_{l} Y_{L}\right) \int_{z_{1}}^{y} d z_{2}[0,1 \mid 1] F_{k j}^{R} \int_{z_{2}}^{y}[0,1 \mid 0] F_{R}^{k i} \\
& +\chi_{L} m S^{(1)}(x, y) \not \xi \xi_{i} \xi^{j} \int_{x}^{y} d z_{1}[0,2 \mid 1] \gamma^{l}\left(D_{l} Y_{L}\right) \int_{z_{1}}^{y} d z_{2}[0,3 \mid 0] F_{k j}^{R} \int_{z_{2}}^{y}[0,1 \mid 0] F_{R}^{k i} \\
& +\chi_{L} m S^{(1)}(x, y) \& \xi_{i} \int_{x}^{y} d z_{1}[0,1 \mid 1] Y_{L} \gamma^{j} F_{m j}^{R} \int_{z_{1}}^{y} d z_{2}[0,1 \mid 0] F_{R}^{m i} \\
& -2 \chi_{L} m S^{(1)}(x, y) \xi_{i} \xi^{j} Y_{L} \int_{x}^{y} d z_{1}[0,1 \mid 1] F_{k j}^{R} \int_{z_{1}}^{y} d z_{2}[0,1 \mid 0] F_{R}^{k i} .
\end{aligned}
$$

The higher orders in the mass matrices are treated similarly. To the order $\mathcal{O}\left((y-x)^{2}\right)$ on the light cone, only the terms up to fourth order in $m Y_{L / R}$ contribute (see (2.95)). One 
gets the formulas

$$
\begin{aligned}
& \chi_{L} m^{2} \sum_{n_{1}, n_{2}, n_{3}=0}^{\infty}\left(\left(-s A_{L}\right)^{n_{1}} s Y_{L} s\left(-A_{R} s\right)^{n_{2}} Y_{R} s\left(-A_{R} s\right)^{n_{3}}\right)(x, y) \\
& \text { phase-free } \frac{i}{2} \chi_{L} m^{2} S^{(0)}(x, y) \& \int_{x}^{y} d z[0,0 \mid 0] Y_{L} Y_{R} \\
& -\frac{i}{2} \chi_{L} m^{2} S^{(1)}(x, y) \& \int_{x}^{y} d z_{1}[0,1 \mid 0] \gamma^{j}\left(D_{j} Y_{L}\right) \int_{z_{1}}^{y} d z_{2}[0,0 \mid 0] \gamma^{k}\left(D_{k} Y_{R}\right) \\
& +\frac{i}{2} \chi_{L} m^{2} S^{(1)}(x, y) \& \int_{x}^{y} d z[0,0 \mid 1] Y_{L}\left(\Delta Y_{R}\right) \\
& +i \chi_{L} m^{2} S^{(1)}(x, y) \& \int_{x}^{y} d z[0,0 \mid 1]\left(D_{i} Y_{L}\right)\left(D^{i} Y_{R}\right) \\
& +\frac{i}{2} \chi_{L} m^{2} S^{(1)}(x, y) \& \int_{x}^{y} d z[0,0 \mid 1]\left(\Delta Y_{L}\right) Y_{R} \\
& +i \chi_{L} m^{2} S^{(1)}(x, y) \int_{x}^{y} d z[0,1 \mid 0] Y_{L} \gamma^{j}\left(D_{j} Y_{R}\right) \\
& +i \chi_{L} m^{2} S^{(1)}(x, y) \int_{x}^{y} d z[0,1 \mid 0] \gamma^{j}\left(D_{j} Y_{L}\right) Y_{R} \\
& -i \chi_{L} m^{2} S^{(1)}(x, y) Y_{L} \int_{x}^{y} d z[0,0 \mid 0] \gamma^{j}\left(D_{j} Y_{R}\right) \\
& -\frac{1}{2} \chi_{L} m^{2} S^{(1)}(x, y) \& \xi^{i} \int_{x}^{y} d z_{1}[0,1 \mid 1] j_{i}^{L} \int_{z_{1}}^{y} d z_{2}[0,0 \mid 0] Y_{L} Y_{R} \\
& +\chi_{L} m^{2} S^{(1)}(x, y) \not \xi \xi^{i} \int_{x}^{y} d z_{1}[0,1 \mid 1] F_{j i}^{L} \int_{z_{1}}^{y} d z_{2}[0,1 \mid 0] Y_{L}\left(D^{j} Y_{R}\right) \\
& +\chi_{L} m^{2} S^{(1)}(x, y) \not \xi \xi^{i} \int_{x}^{y} d z_{1}[0,1 \mid 1] F_{j i}^{L} \int_{z_{1}}^{y} d z_{2}[0,1 \mid 0]\left(D^{j} Y_{L}\right) Y_{R} \\
& +\frac{1}{4} \chi_{L} m^{2} S^{(1)}(x, y) \not \int_{x}^{y} d z_{1}[0,1 \mid 0] \gamma^{j} \gamma^{k} F_{j k}^{L} \int_{z_{1}}^{y} d z_{2}[0,0 \mid 0] Y_{L} Y_{R} \\
& +\chi_{L} m^{2} S^{(1)}(x, y) \xi^{i} \int_{x}^{y} d z_{1}[0,2 \mid 0] \gamma^{j} F_{j i}^{L} \int_{z_{1}}^{y} d z_{2}[0,0 \mid 0] Y_{L} Y_{R} \\
& -\frac{1}{2} \chi_{L} m^{2} S^{(1)}(x, y) \& \xi^{i} \int_{x}^{y} d z_{1}[0,2 \mid 0] Y_{L} Y_{R} \int_{z_{1}}^{y} d z_{2}[0,0 \mid 1] j_{i}^{L} \\
& -\frac{1}{2} \chi_{L} m^{2} S^{(1)}(x, y) \& \xi^{i} \int_{x}^{y} d z_{1}[0,1 \mid 1] Y_{L} Y_{R} \int_{z_{1}}^{y} d z_{2}[0,2 \mid 0] j_{i}^{L} \\
& +\chi_{L} m^{2} S^{(1)}(x, y) \not \& \xi^{i} \int_{x}^{y} d z_{1}[0,1 \mid 1] Y_{L}\left(D^{j} Y_{R}\right) \int_{z_{1}}^{y} d z_{2}[0,1 \mid 0] F_{j i}^{L} \\
& +\chi_{L} m^{2} S^{(1)}(x, y) \not \xi \xi^{i} \int_{x}^{y} d z_{1}[0,1 \mid 1]\left(D^{j} Y_{L}\right) Y_{R} \int_{z_{1}}^{y} d z_{2}[0,1 \mid 0] F_{j i}^{L} \\
& +\frac{1}{4} \chi_{L} m^{2} S^{(1)}(x, y) \not \int_{x}^{y} d z_{1}[0,1 \mid 0] Y_{L} Y_{R} \int_{z_{1}}^{y} d z_{2}[0,0 \mid 0] \gamma^{j} \gamma^{k} F_{j k}^{L} \\
& +\chi_{L} m^{2} S^{(1)}(x, y) \xi^{i} \int_{x}^{y} d z_{1}[0,2 \mid 0] Y_{L} Y_{R} \int_{z_{1}}^{y} d z_{2}[0,1 \mid 0] \gamma^{j} F_{j i}^{L}+\mathcal{O}\left((y-x)^{2}\right) \\
& \chi_{L} m^{3} \sum_{n_{1}, n_{2}, n_{3}, n_{4}=0}^{\infty}\left(\left(-s A_{L}\right)^{n_{1}} s Y_{L} s\left(-A_{R} s\right)^{n_{2}} Y_{R} s\left(-A_{L} s\right)^{n_{3}} Y_{L} s\left(-A_{R} s\right)^{n_{4}}\right)(x, y) \\
& \overbrace{}^{\text {phase-free }} \chi_{L} m^{3} S^{(1)}(x, y) Y_{L} \int_{x}^{y} d z[0,0 \mid 0] Y_{R} Y_{L}
\end{aligned}
$$




$$
\begin{aligned}
& \quad+\frac{1}{2} \chi_{L} m^{3} S^{(1)}(x, y) \not \int_{x}^{y} d z_{1}[0,1 \mid 0] Y_{L} Y_{R} \int_{z_{1}}^{y} d z_{2}[0,0 \mid 0] \gamma^{j}\left(D_{j} Y_{L}\right) \\
& +\frac{1}{2} \chi_{L} m^{3} S^{(1)}(x, y) \not \int_{x}^{y} d z_{1}[0,1 \mid 0] \gamma^{j}\left(D_{j} Y_{L}\right) \int_{z_{1}}^{y} d z_{2}[0,0 \mid 0] Y_{R} Y_{L}+\mathcal{O}\left((y-x)^{2}\right) \\
& \chi_{L} m^{4} \sum_{n_{1}, \ldots, n_{5}=0}^{\infty}\left(\left(-s A_{L}\right)^{n_{1}} s Y_{L} s\left(-A_{R} s\right)^{n_{2}}\right. \\
& \left.\quad \times Y_{R} s\left(-A_{L} s\right)^{n_{3}} Y_{L} s\left(-A_{R} s\right)^{n_{4}} Y_{R} s\left(-A_{L} s\right)^{n_{5}}\right)(x, y) \\
& \underset{\text { phase-free }}{\simeq} \frac{i}{2} \chi_{L} m^{4} S^{(1)}(x, y) \not{\&} \int_{x}^{y} d z_{1}[0,1 \mid 0] Y_{L} Y_{R} \int_{z_{1}}^{y} d z_{2}[0,0 \mid 0] Y_{L} Y_{R}+\mathcal{O}\left((y-x)^{2}\right)
\end{aligned}
$$

These classes of Feynman diagrams completely characterize the Green's functions to the order $\mathcal{O}\left((y-x)^{2}\right)$ on the light cone; notice that we only get a finite number of contributions.

\section{References}

[1] F. Finster, "Definition of the Dirac sea in the presence of external fields," hepth/9705006, Adv. Theor. Math. Phys. 2 (1998) 963-985

[2] F. Finster, "Light-cone expansion of the Dirac sea to first order in the external potential," hep-th/9707128, Mich. J. Math. 46 (1999) 377-408

[3] F. Finster, "Light-Cone expansion of the Dirac sea with light-cone integrals," functan/9707003 (unpublished preprint) 